\title{
Classification of 3 + 1D Bosonic Topological Orders (II): The Case When Some Pointlike Excitations Are Fermions
}

\author{
Tian Lan ${ }^{1}$ and Xiao-Gang Wen ${ }^{2}$ \\ ${ }^{1}$ Institute for Quantum Computing, University of Waterloo, Waterloo, Ontario N2L 3G1, Canada \\ ${ }^{2}$ Department of Physics, Massachusetts Institute of Technology, Cambridge, Massachusetts 02139, USA
}

(Received 3 August 2018; revised manuscript received 1 February 2019; published 10 April 2019; corrected 12 April 2019)

\begin{abstract}
Topological orders describe a new class of gapped quantum phases of matter at zero temperature, that has various patterns of many-body quantum entanglement. Previously, topological orders in one and two spatial dimensions have been systematically understood and classified. This paper [together with Phys. Rev. X 8, 021074 (2018)] develops a systematic and classifying understanding of topological orders in three-dimensional bosonic systems, where the number of topological types for the pointlike and stringlike excitations is assumed to be finite. Our systematic understanding comes from the unique canonical boundary for each $3+1 \mathrm{D}$ topological order. We find that the pointlike and stringlike excitations on the canonical boundary are described fully by a mathematical theory-the so-called fusion 2-categories. This theory allows us to classify $3+1 \mathrm{D}$ topological orders in bosonic systems in terms of a subset of fusion 2-categories. This systematic understanding further leads to a systematic understanding of $3+1 \mathrm{D}$ topological orders in bosonic and fermionic systems with arbitrary finite unitary symmetry.
\end{abstract}

DOI: 10.1103/PhysRevX.9.021005

Subject Areas: Condensed Matter Physics, Strongly Correlated Materials

\section{INTRODUCTION}

The study of topological phases of matter has become a very active field in condensed-matter physics. They can be divided into two classes. The first class is the topologically ordered states realized in strongly interacting quantum Hall systems [1] and some strongly interacting quantum spinliquid materials [2-6]. The notion of topological order was introduced in 1989 [7,8], and now we realize that topological orders are nothing but the patterns of many-body entanglement [9]. Topological orders have pointlike and/or stringlike excitations, which have nontrivial topological properties (such as braiding statistics), as well as highly nontrivial boundary properties. Those properties are robust against any local perturbations. The robustness against arbitrary local perturbations is the meaning of topological in the name topological order.

The second class of topological phases of matter, such as topological insulators [10-16], can be described by the noninteracting band theory, which is very familiar to condensed-matter physicists. This class of topological phases also has highly nontrivial boundary properties, but no topological excitations. The nontrivial boundary

Published by the American Physical Society under the terms of the Creative Commons Attribution 4.0 International license. Further distribution of this work must maintain attribution to the author(s) and the published article's title, journal citation, and DOI. properties require protection by some symmetries. They are not robust against symmetry-breaking local perturbations; on the contrary, the robust properties of the first class survive even when all the symmetries are broken. The topological insulators are noninteracting examples of symmetry-protected topological (SPT) orders [17-20] (also known as symmetry-protected trivial orders [21]).

This paper is about the first class of topological phases of matter-topological orders [22] - in three-dimensional bosonic systems. Previously, it was shown that there is no topological order in one-dimensional bosonic systems $[23,24]$. The topological orders in two-dimensional bosonic and fermionic systems are also understood systematically in terms of the tensor category theory [25-29]. The abstract mathematical tensor category theory is nothing but a detailed theory to describe topological pointlike excitations.

We remark that many-body entanglement (i.e., topological order) is a totally new phenomenon. Usually, to understand a truly new phenomenon, we need to introduce new languages and new mathematical framework. Historically, we have introduced group and its representation theory into physics to describe symmetries in quantum systems. Now, we need to introduce category and higher category theory into physics to describe many-body entanglement.

The study and the systematic understanding of many-body entanglement (i.e., topological order) has a wide and deep impact in physics. Topological order reveals a new class of quantum matter that enables a new kind of quantum computing-topological quantum computing 
[26,30-32]. Topological order also reveals the quantum information origin of elementary particles and fundamental forces [33-36].

However, until not long ago, we lacked a systematic understanding of topological orders in three-dimensional systems. Reference [37] started an attempt to have a systematic and classifying understanding of $3+1 \mathrm{D}$ topological orders. This paper is a sequel to Ref. [37].

In Ref. [37], we classified the so-called all-boson (AB) $3+1 \mathrm{D}$ topological orders - the $3+1 \mathrm{D}$ topological orders whose emergent pointlike excitations are all bosons. We found that all $3+1 \mathrm{D}$ AB topological orders are classified by pointed unitary fusion 2-categories with trivial 1-morphisms, which are one-to-one labeled by a pair $\left(G, \omega_{4}\right)$ up to group automorphisms, where $G$ is a finite group and $\omega_{4}$ its group 4-cohomology class: $\omega_{4} \in H^{4}(G ; \mathbb{R} / \mathbb{Z})$.

In this paper, we classify $3+1 \mathrm{D}$ topological orders whose emergent pointlike excitations are bosons and fermions, which are called EF topological orders. The results in Ref. [37] and in this paper classify all 3+1D topological orders in bosonic systems, since there are only two kinds of statistics for pointlike excitations in $3+1 \mathrm{D}$ : Bose statistics and Fermi statistics. This result, in turn, leads to a classification of $3+1 \mathrm{D}$ topological orders with finite unitary symmetry for bosonic and fermionic systems, which include 3+1D SPT orders with finite unitary symmetry for bosonic and fermionic systems (see Sec. IX). In addition, we argue that all $3+1 \mathrm{D}$ bosonic topological orders always have a gappable boundary.

The pointlike excitations and the stringlike excitations in $3+1 \mathrm{D}$ bosonic topological orders [38-50] can fuse and braid, and their fusion and braiding must form a selfconsistent structure. In particular, the self-consistent structure must satisfy the principle of remote detectability [51,52]: A topological order is anomaly-free, if and only if every nontrivial topological excitation can be detected by other topological excitations via some remote operation. This principle is called the anomaly-free condition in Ref. [52]. Here, "nontrivial topological excitation" means an excitation with a nontrivial type as defined in Sec. III B. "Anomaly-free" means realizable by a local bosonic lattice model in the same dimension [53].

Since the remote detection is done by braiding, the self-consistency of fusion and braiding plus the remote detectability can totally fix the structure of pointlike and stringlike excitations. Those structures, in turn, classify the $3+1 \mathrm{D}$ EF topological orders.

\section{SUMMARY OF RESULTS}

\section{A. A list of the results obtained in this paper}

In this paper, we classify EF topological orders for $3+1 \mathrm{D}$ bosonic systems where some emergent pointlike excitations are fermions. We assume the numbers of different topological types for the pointlike and stringlike excitations to be finite. To understand our result, a minimal understanding of 2-category is required. The $2+1 \mathrm{D}$ topological order is mathematically described by (braided) fusion categories, whose objects correspond to pointlike excitations (anyons), and morphisms (or 1-morphisms in the context of higher category theory) correspond to physical processes, such as braiding. In $3+1 \mathrm{D}$ topological order, in addition to pointlike excitations, we have stringlike excitations; the categorical level is increased by one, and we need (braided) fusion 2-categories. We conclude in Table I the physical meanings of the commonly used 2-category terms in this paper for the reader's reference.

Here, we first list the main results. In the following subsections, we explain those results in more physical terms.

(1) We argue that all $3+1 \mathrm{D}$ topological orders for bosonic systems have a gappable boundary.

(2) All the pointlike excitations in EF topological orders are described by the representations of $G_{f}=$ $Z_{2}^{f} \lambda_{e_{2}} G_{b}-\mathrm{a} Z_{2}^{f}$ central extension of a finite group $G_{b}$ characterized by a group cocycle $e_{2} \in H^{2}\left(G_{b}, \mathbb{Z}_{2}\right)$.

(3) We find that every EF topological order one to one corresponds a $2+1 \mathrm{D}$ anomalous topological order $\mathcal{A}_{b}^{3}$ on its unique canonical boundary, where the $3+1 \mathrm{D}$ EF topological order is given by the bulk center $Z\left(\mathcal{A}_{b}^{3}\right)$, which is the generalization of a Drinfeld center to higher dimensions [52,54,55]. Here, $\mathcal{A}_{b}^{3}$ is a unitary fusion 2-category with simple objects labeled by $\hat{G}_{b}=Z_{2}^{m} \lambda_{m_{2}} G_{b}, m_{2} \in H^{2}\left(G_{b}, Z_{2}\right) . \mathcal{A}_{b}^{3}$ also has one invertible fermionic 1-morphism for each object as well as quantum-dimension- $\sqrt{2}$

TABLE I. Physical meanings of categorical terms.

\begin{tabular}{lcl}
\hline \hline 2-category & Notation & \multicolumn{1}{c}{ Physical meaning } \\
\hline Object (0-morphism) & $\mathbf{1}$ (trivial), $s, g, h, \ldots$ & Stringlike excitation \\
1-morphism & $\operatorname{Hom}(\mathbf{1}, \mathbf{1})$ & Pointlike excitations \\
& $\operatorname{Hom}(s, s)$ & Pointlike excitations on string $s$ \\
& $\operatorname{Hom}(g, h)$ & Pointlike domain wall between string $g$ and $h$ \\
2-morphism & & Physical operators \\
\hline \hline
\end{tabular}


1-morphisms that connect two objects $g$ and $g m$, where $g \in \hat{G}_{b}$ and $m$ is the generator of $Z_{2}^{m}$.

(4) When $\hat{G}_{b}=Z_{2}^{m} \times G_{b}$, the EF topological orders are called EF1 topological orders, which is classified by simple data $\left(G_{b}, e_{2}, n_{3}, \nu_{4}\right)$, where $n_{3} \in H^{3}\left(G_{b}, \mathbb{Z}_{2}\right)$ and $\nu_{4}$ is a 4-cochain in $C^{4}\left(G_{b}, U(1)\right)$ satisfying $\mathrm{d} \nu_{4}=(-)^{n_{3} \smile n_{3}+e_{2}-n_{3}}$.

(5) When $\hat{G}_{b}$ is a nontrivial $Z_{2}^{m}$ extension [i.e., when $m_{2}$ is a nontrivial group cocycle in $\left.H^{2}\left(G_{b}, Z_{2}\right)\right]$, the EF topological orders are called EF2 topological orders, where some junctions of three stringlike excitations must carry Majorana zero modes.

(6) Every EF2 topological order with $G_{f}=Z_{2}^{f} \lambda G_{b}$ can be associated with an EF1 topological order with $G_{f}=Z_{2}^{f} \lambda \hat{G}_{b}$, which may lead to an understanding of EF2 topological orders in terms of simpler EF1 topological orders.

(7) We find that all EF topological orders correspond to gauged $3+1 \mathrm{D}$ fermionic SPT orders with a finite unitary symmetry group. Our results can also be viewed as a classification of the corresponding $3+1 \mathrm{D}$ fermionic SPT orders.

(8) We further propose that the general classification of $3+1 \mathrm{D}$ topological orders with finite unitary symmetries for bosonic and fermionic systems can be obtained by gauging or partially gauging the finite symmetry group of $3+1 \mathrm{D}$ SPT phases of bosonic and fermionic systems.

\section{B. Emergence of a group $\boldsymbol{G}_{f}$}

Since there is no braiding statistics in three-dimensional space, the pointlike excitations are described by a symmetric fusion category s $\mathcal{R e p}\left(G_{f}\right)$ [56]. In other words, each type of pointlike excitation corresponds to an irreducible representation of a finite group $G_{f}$. The quantum dimension of the excitations is given by the dimension of the representation. $G_{f}$ is a $Z_{2}^{f}$ central extension of $G_{b}$ :

$$
1 \rightarrow Z_{2}^{f} \rightarrow G_{f} \stackrel{\pi^{f}}{\rightarrow} G_{b} \rightarrow 1 .
$$

A pointlike excitation may correspond to a representation where the subgroup $Z_{2}^{f}$ is represented trivially. Such a pointlike excitation is a boson. On the other hand, if $Z_{2}^{f}$ is represented nontrivially, the corresponding pointlike excitation is a fermion.

\section{Unique canonical gapped boundary described by a unitary fusion 2-category}

Following a similar approach proposed in Ref. [37], in this paper, we show that every EF topological order has a unique canonical gapped boundary, which is described by a unitary fusion 2-category $\mathcal{A}_{b}^{3}$. Let us describe such fusion 2-categories in detail. The simple objects of the fusion 2-category, corresponding to the boundary strings, are labeled by $\hat{G}_{b}$. Here, $\hat{G}_{b}$ is an extension of $G_{b}$ by $Z_{2}^{m}$ :

$$
1 \rightarrow Z_{2}^{m} \rightarrow \hat{G}_{b} \stackrel{\pi^{m}}{\rightarrow} G_{b} \rightarrow 1 .
$$

The fusion of those boundary strings (the objects) is described by the group multiplication of $\hat{G}_{b}$.

In the fusion 2-category, there is a nontrivial 1-morphism of unit quantum dimension that connects each simple object $g$ to itself. Such a 1-morphism corresponds to a pointlike topological excitation living on the string $g$. Since it can live on a trivial string labeled by $g=1$, these pointlike excitations are actually not confined to certain strings; they can move freely on the boundary and braid among themselves. The statistics of this pointlike excitation (the 1-morphism) is fermionic. So the canonical boundary of an EF topological order also contains a fermion in addition to the boundary strings.

There is also a 1-morphism of quantum dimension $\sqrt{2}$ that connects object $g$ to object $g m$, where $m$ is the generator of $Z_{2}^{m}$. Physically, it means that the domain wall between string $g$ and string $g m$ carries a fractional degree of freedom (d.o.f.) of dimension $\sqrt{2}$ (i.e., like one-half of a qubit). There are no other simple 1-morphisms.

For simplicity, we refer to the unitary fusion 2-categories with the above special properties as EF 2-categories. In this paper, we show that each EF topological order corresponds to an EF 2-category. Reference [57] shows that, for each of EF 2-categories, one can construct a bosonic model to realize an EF topological order that has a boundary described by the EF 2-category. Thus, the classification of EF 2-categories corresponds to a classification of $3+1 \mathrm{D} \mathrm{EF}$ topological orders. We mention that Ref. [58] also gave explicit constructions of bosonic models to realize $3+1 \mathrm{D}$ EF topological orders for the cases of Abelian $G_{b}$, in terms of twisted Crane-Yetter models [59-62].

We note that the boundary fermion can form a topological $p$-wave superconducting (pSC) chain [63,64], which is the nontrivial phase in Kitaev's Majorana chain model [63]. In fact, two boundary strings labeled by $g$ and gm differ by attaching such a pSC chain. The 1-morphism of quantum dimension $\sqrt{2}$ at the domain wall between the strings $g$ and $g m$ is nothing but the Majorana zero mode at the end of the pSC chain.

\section{Emergence of Majorana zero modes}

The above classification of EF topological orders allows us to divide those EF topological orders into EF1 topological orders when $\hat{G}_{b}=Z_{2}^{m} \times G_{b}$ and EF2 topological orders when $\hat{G}_{b}$ is a nontrivial $Z_{2}^{m}$ extension of $G_{b}$, described by a group 2-cocycle $m_{2}\left(g_{b}, h_{b}\right) \in H^{2}\left(G_{b}, Z_{2}\right)$. In the following, we describe how to directly measure the group 2-cocycle $m_{2}$ via the Majorana zero modes carried by the junctions of three strings. 


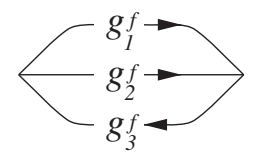

FIG. 1. A string configuration in the bulk described by a triple $\left(\chi_{g_{1}^{f}}, \chi_{g_{2}^{f}}, \chi_{g_{3}^{f}}\right)$, where $\chi_{g^{f}}$ is a conjugacy class in $G_{f}$ containing $g^{f} \in G_{f}$ and the triple satisfies $g_{1}^{f} g_{2}^{f}=g_{3}^{f}$.

Consider a fixed set of strings labeled by $\chi_{g^{f}}$, where $\chi_{g^{f}}$ is a conjugacy class in $G_{f}$ that containing $g^{f} \in G_{f}$. Three strings $\chi_{g_{1}^{f}}, \chi_{g_{2}^{f}}$, and $\chi_{g_{3}^{f}}$ can annihilate if $g_{1}^{f} g_{2}^{f}=g_{3}^{f}$. If the triple string junction has a Majorana zero mode, we assign $m_{2}^{f}\left(g_{1}^{f}, g_{2}^{f}\right)=-1$. If the triple string junction has no Majorana zero mode, we assign $m_{2}^{f}\left(g_{1}^{f}, g_{2}^{f}\right)=1$. (When $G_{f}$ is Abelian, the appearance of Majorana zero modes can be determined by the twofold topological degeneracy for the configuration in Fig. 1.) $m_{2}^{f}\left(g_{1}^{f}, g_{2}^{f}\right)$ depends only on the conjugacy classes of $g_{1}^{f}, g_{2}^{f}$, and $g_{3}^{f}$. Thus, $m_{2}^{f}$ satisfies

$m_{2}^{f}\left(g_{1}^{f}, g_{2}^{f}\right)=m_{2}^{f}\left(h_{1} g_{1}^{f} h_{1}^{-1}, h_{2} g_{2}^{f} h_{2}^{-1}\right), \quad h_{1}, h_{2} \in G_{f}$.

Under proper choices of the fixed set of strings (see Sec. VIII E), $m_{2}^{f}\left(g_{1}^{f}, g_{2}^{f}\right)$ is a function on $G_{b}$; i.e., it has the form

$$
m_{2}^{f}\left(g_{1}^{f}, g_{2}^{f}\right)=\tilde{m}_{2}\left[\pi^{f}\left(g_{1}^{f}\right), \pi^{f}\left(g_{2}^{f}\right)\right]
$$

$\tilde{m}_{2}$ in the above is cohomologically equivalent to $m_{2}$ that describes the extension $\hat{G}_{b}$; in other words, we measure $m_{2}$ up to coboundaries. If the measured $m_{2}$ is trivial in $H^{2}\left(G_{b}, Z_{2}\right)$, the corresponding bulk topological order is an EF1 topological order. If the measured $m_{2}$ is a nontrivial cocycle, we get an EF2 topological order. We emphasize that, for EF1 topological orders, emergent Majorana zero modes can be removed by changing the fixed set of strings, while, for EF2 topological orders, the appearance of emergent Majorana zero modes is inevitable.

We mention that, besides the junctions of three strings, emergent Majorana zero modes may also be carried by linked loops as first proposed in Ref. [65]. Appendix B discuss the relation between the 2-cocycle $m_{2}$ and the Majorana zero modes on the linked loops.

\section{E. Classification of EF1 topological order by a class of pointed unitary fusion 2-category}

For an EF1 topological order, the unitary fusion 2-category that describes its canonical boundary can be simplified, since we can treat the pSC chain as a trivial string when $\hat{G}_{b}=Z_{2}^{m} \times G_{b}$. The simplified unitary fusion 2-category $\overline{\mathcal{A}}_{b}^{3}$ has simple objects labeled by $G_{b}$ and a fermionic 1-morphism of unit quantum dimension that connects each simple object to itself. There are no simple noninvertible 1 -morphisms. Thus, $\overline{\mathcal{A}}_{b}^{3}$ is a pointed unitary fusion 2-category. We study this case thoroughly and show that $\overline{\mathcal{A}}_{b}^{3}$ are classified by data $\left(G_{b}, e_{2}, n_{3}, \nu_{4}\right)$, where $G_{b}=G_{f} / Z_{2}^{f}, e_{2} \in H^{2}\left(G_{b}, \mathbb{Z}_{2}\right)$ is the 2-cocycle determining the extension $Z_{2}^{f} \rightarrow G_{f} \rightarrow G_{b}, n_{3} \in H^{3}\left(G_{b}, \mathbb{Z}_{2}\right)$, and $\nu_{4}$ is a 4-cochain in $C^{4}\left[G_{b}, U(1)\right]$ satisfying

$$
d \nu_{4}=(-)^{n_{3} \smile n_{3}+e_{2}-n_{3}} .
$$

Here, $\underset{k}{ }$ denotes the higher cup product which is defined in Ref. [66]. The above data $\left(G_{b}, e_{2}, n_{3}, \nu_{4}\right)$ classify the EF1 topological orders. This result is closely related to a partial classification of fermionic SPT phases [67], where a similar twisted cocycle condition (5) was first obtained (without the $e_{2} \smile n_{3}$ term). Equation (5) is also discussed in Ref. [58], for the cases when $G_{b}$ is Abelian, and in Ref. [68] for arbitrary finite $G_{b}$ (as well as a generalization to any dimensions).

In Ref. [57], we give explicit constructions and show that all such pointed unitary fusion 2-categories correspond to $3+1 \mathrm{D} \mathrm{EF}$ topological orders. In fact, all $3+1 \mathrm{D}$ EF1 topological orders can be realized [57] by 2-gauge theories [62].

\section{F. A map from EF topological orders to EF1 topological orders}

Let us now shift our attention from EF1 topological orders to general EF topological orders. Although general EF 2-categories are more complicated, we can nevertheless obtain some understanding of them by constructing, for any general EF 2-category $\mathcal{A}_{b}^{3}$ defined in Sec. II C, an associated pointed unitary fusion 2-category $\tilde{\mathcal{A}}_{b}^{3}$, which is just the pointed sub-2-category of $\mathcal{A}_{b}^{3}$. In $\tilde{\mathcal{A}}_{b}^{3}$, the quantumdimension- $\sqrt{2}$ 1-morphisms are thrown away, but the simple objects remain as $\hat{G}_{b}$. Note that $\tilde{\mathcal{A}}_{b}^{3}$ should be distinguished from $\overline{\mathcal{A}}_{b}^{3}$, where not only the quantumdimension- $\sqrt{2}$ 1-morphisms, but also the pSC chains, are thrown away, and simple objects become the smaller $G_{b}$. Throwing away pSC chains is possible only when $\hat{G}_{b}=G_{b} \times Z_{2}^{m}$, i.e., the EF1 case. Thus, there is a map from the EF 2-categories $\mathcal{A}_{b}^{3}$ to the pointed unitary fusion 2-categories $\tilde{\mathcal{A}}_{b}^{3}$. In other words, there is a map from EF topological orders to EF1 topological orders. This relation may make it possible to construct a generic EF topological order from a simpler EF1 topological order by adding some additional structure. 


\section{G. A general classification of 3+1D topological orders with finite unitary symmetry for bosonic and fermionic systems}

With the above classification results, we further propose that the general classification of $3+1 \mathrm{D}$ topological orders with symmetries can be obtained by gauging $3+1 \mathrm{D}$ SPT phases. Partially gauging a SPT phase leads to a phase with both topological order and symmetry, namely, a symmetryenriched topological (SET) phase, while fully gauging the symmetry leads to an intrinsic topological order. In the same gauging sequence, the starting SPT phase, the partially gauged intermediate SET phases, and the ending topological order share the same classification data; the equivalence relations for SPT, SET, and topological order can be different, though. See Sec. IX for a more detailed discussion.

\section{H. The line of arguments}

The key result of this paper, the classification of $3+1 \mathrm{D}$ EF topological orders, is obtained via the following line of arguments.

(1) In Sec. III, we show that condensing all the bosonic pointlike excitations in a $3+1 \mathrm{D}$ EF topological order always gives rise to a unique $Z_{2}^{f}$ topological order.

(2) In Sec. IV, we argue that the domain wall between the $\mathrm{EF}$ and the $Z_{2}^{f}$ topological orders, induced by the condensation of all the bosonic pointlike excitations, can be gapped. Since there is a canonical gapped boundary of the $Z_{2}^{f}$ topological order, we obtain a unique canonical gapped boundary of every $3+1 \mathrm{D}$ EF topological order.

(3) Then, in Secs. V and VI, we further study the properties of the domain wall and the boundary. We show that they have strings whose fusion is described by group multiplication, also a fermionic pointlike excitation and a Majorana zero mode at the junction of certain pairs of strings. Thus, the domain wall and the boundary are described by unitary fusion 2-categories with certain special properties, which are discussed in more detail in Secs. VII and VIII.

(4) By the principle that boundary uniquely determines bulk [52,54,55], the above leads to a classification of $3+1 \mathrm{D}$ EF topological orders in terms of a subclass of unitary fusion 2-categories.

\section{CONDENSING ALL THE BOSONIC POINTLIKE EXCITATIONS TO OBTAIN A UNIQUE $Z_{2}^{f}$ TOPOLOGICAL ORDER}

Some pointlike excitations in a $3+1 \mathrm{D}$ EF topological order are bosons, and the others are fermions. In this section, we show that, by condensing all the bosonic pointlike excitations, we will always ends up with a simple

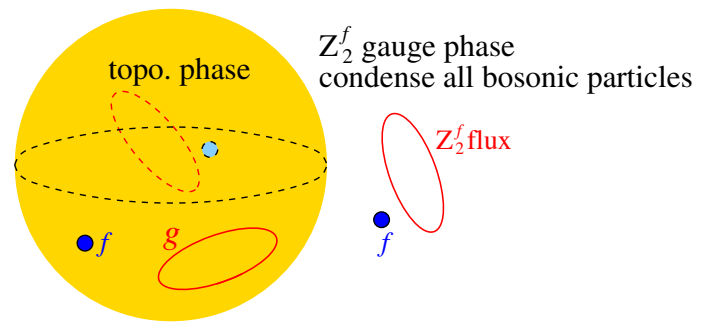

FIG. 2. Condensing all bosonic pointlike excitations in a $3+1 \mathrm{D} \mathrm{EF}$ topological order $\mathcal{C}_{\mathrm{EF}}^{4}$ gives rise to $3+1 \mathrm{D} Z_{2}^{f}$ topological order $\mathcal{C}_{Z_{2}^{f}}^{4}$. $\mathcal{C}_{\mathrm{EF}}^{4}$ contain a fermionic pointlike excitation $f$ and a stringlike excitation, $Z_{2}^{f}$ flux, which behave like the $\pi$-flux line for the fermion $f$. The domain wall $\mathcal{A}_{w}^{3}$ between $\mathcal{C}_{\mathrm{EF}}^{4}$ and $\mathcal{C}_{Z_{2}^{f}}^{4}$ contain strings labeled by elements $g \in G_{f}$ and only one fermionic particle $f$. The strings and the fermion have quantum dimension 1.

$Z_{2}^{f}$ topological order-a topological order described by the $3+1 \mathrm{D} Z_{2}$-gauge theory, but with a fermionic $Z_{2}$ charge [38] (see Fig. 2). In the next few subsections, we introduce related concepts and pictures that allow us to obtain such a result.

\section{A. Pointlike excitations and group structure in 3+1D EF topological orders}

The pointlike excitations in $3+1 \mathrm{D}$ EF topological orders are described by the symmetric fusion category. According to Tannaka duality (see Appendix A), the symmetric fusion category gives rise to a group $G_{f}$ such that the pointlike excitations are labeled by the irreducible representations of $G_{f}$. In addition, $G_{f}$ contains a $Z_{2}$ central subgroup, denoted by $Z_{2}^{f}=\{\mathbf{1}, z\}$. In each irreducible representation of $G_{f}, z$ is represented by either $I$ or $-I$ (where $I$ is an identity matrix). If $z=I$, the corresponding pointlike excitation is a boson. We note that all the bosonic pointlike excitations are described by representations of $G_{b}, \mathcal{R e p}\left(G_{b}\right)$, where $G_{b}=G_{f} / Z_{2}^{f}$. If $z=-I$, the corresponding pointlike excitation is a fermion. We denote such a symmetric fusion category by $\operatorname{s} \mathcal{R} \operatorname{ep}\left(G_{f}\right)$. In addition, irreducible representations correspond to simple pointlike excitations, while reducible representations correspond to nonsimple, or composite, pointlike excitations. Composite excitations always split as the direct sum of simple ones.

\section{B. Stringlike excitations in $3+1 \mathrm{D}$ EF topological orders}

The pointlike excitations have trivial mutual statistics among them. One cannot use the pointlike excitations to detect other pointlike excitations by remote operations. Thus, based on the principle of remote detectability, there must be stringlike excitations in $3+1 \mathrm{D}$ EF topological orders, so that every pointlike excitation can be detected 
by some stringlike excitations via remote braiding. Similarly, every stringlike excitation can be detected by some pointlike and/or stringlike excitations via remote braiding. We see that the properties of stringlike excitations are determined by the pointlike topological excitations [i.e., $\left.\operatorname{s} \mathcal{R e p}\left(G_{f}\right)\right]$ to a certain degree.

Let us discuss some basic properties of stringlike excitations. First, similar to the particle case (see Appendix A), a stringlike excitation $s_{i}$ can be defined via a trap Hamiltonian $\Delta H_{\text {str }}\left(s_{i}\right)$ which is nonzero along a loop. Such a trap Hamiltonian $\Delta H_{\text {str }}\left(s_{i}\right)$ can be understood as modifying the original Hamiltonian $H_{0}$, by adding a potential energy configuration that makes it energetically favorable to trap a topologically nontrivial excitation in a certain location, so that it becomes the ground state of the modified Hamiltonian. The ground-state subspace of total Hamiltonian $H_{0}+\sum_{i} \Delta H_{\text {str }}\left(s_{i}\right)$ defines the fusion space of strings $s_{i}$ [and particles $p_{i}$ if we also have particle traps $\left.\Delta H\left(p_{i}\right)\right]: \mathcal{V}\left(M, p_{1}, p_{2}, \ldots, s_{1}, s_{2}, \ldots\right)$. We note that such a definition relies on an assumption that all the on-string excitations are gapped. We argue that such is always the case in Ref. [37] provided that the number of topological types for strings is finite.

It is important to first clarify what are the "fundamental," or simple, strings: A stringlike excitation $s_{i}$ is called simple if its fusion space cannot be split by any nonlocal perturbations along the string; i.e., the ground-state degeneracy cannot be split by any nonlocal perturbations of $\Delta H_{\text {str }}\left(s_{i}\right)$. We stress that here we allow nonlocal perturbations which are nonzero only along the string. In addition, we say that two simple strings are of the same type if they can be deformed into each other without closing the energy gap by nonlocal perturbations along the string. The motivation to use nonlocal perturbations is that we want to separate out the degeneracy that is "distributed" between strings and particles.

For example, in a $3+1 \mathrm{D} Z_{2}$-gauge theory, the $Z_{2}$-gauge charge has a mod 2 conservation. Those $Z_{2}$ charges can form a many-body state along a large loop, that spontaneously breaks the mod 2 conservation which leads to a twofold degeneracy. We do not want to regard such a string as a nontrivial simple string. One way to remove such kinds of string as a nontrivial simple string is to require stability against nonlocal perturbations along a simple string. Mathematically, if we allow nonlocal perturbations as morphisms, the above string from $Z_{2}$-charge condensation becomes a direct sum of two trivial strings.

The fusion of simple strings may give us nonsimple strings which can be written as a direct sum of simple strings:

$$
s_{i} \otimes s_{j}=\bigoplus_{k} M_{k}^{i j} s_{k}
$$

Strings, when they are contractable loops, can also shrink to a point and become pointlike excitations:

$$
s_{i} \rightarrow \bigoplus_{j} L_{j}^{i} p_{j}
$$

We say that a string is pure if and only if its shrinking contains trivial pointlike excitation $\mathbf{1}$. A nonpure string can be viewed as a bound state of a pure string with some topological pointlike excitations.

Using $M_{k}^{i j}$ together with shrinking, we can calculate the dimension of fusion space of unlinked loops, as well as the quantum dimension $d_{i}$ of the $s_{i}$ string. Here, $d_{i}$ is defined similarly as that for pointlike excitations [see Eq. (A16)]; it is the effective dimension of "internal d.o.f." of the excitation at a large excitation number limit. More precisely, let $\mathcal{V}\left(S^{3}, s_{i}^{\otimes n}\right)$ be the fusion space of $n$ unlinked loops $s_{i}$, and we have $d_{i}=\lim _{n \rightarrow \infty}\left[\operatorname{dim} \mathcal{V}\left(S^{3}, s_{i}^{\otimes n}\right)\right]^{1 / n}$. A string $s$ with quantum dimension 1 is always simple. Such a string is called invertible or pointed; i.e., there exists another string $s^{\prime}$ such that

$$
s \otimes s^{\prime}=s^{\prime} \otimes s=\mathbf{1} .
$$

Since, in general, it is not easy to determine whether a string is simple by directly checking whether its fusion space can be split or not, in the following we try to make use of the shrinking operation to do the job. In fact, not only strings have a shrinking operation; particles also have a shrinking operation. We note that a zero-dimension sphere $S^{0}$ is two points, which may correspond to a pair of particles $\left(p_{1}, p_{2}\right)$. In higher dimensions, we may have excitations described by $S^{d}$. For $d=0,1,2, \ldots$, they correspond to a pair of particles $\left(p_{1}, p_{2}\right)$, a loop excitation $s$, a spherical membrane excitation $m$, etc. Those excitations are pure if their shrinking contains 1 . For example, an $S^{0}$ excitation $\left(p_{1}, p_{2}\right)$ is pure if and only if $p_{2}$ is the antiparticle of $p_{1}$.

There is a well-known result that $p$ is simple if and only if the shrinking of $p$ and $\bar{p}$ (i.e., the fusion of $p$ and $\bar{p}$ ) contains only a single trivial particle $\mathbf{1}$. In this case, we also say that the corresponding pure $S^{0}$ excitation $(p, \bar{p})$ is simple. By analog, we conjecture a similar condition for higher-dimensional excitations: A pure $S^{d}$ excitation is simple if and only if its shrinking contains only one trivial particle 1. Thus, the shrinking of a pure simple string $s$ must have the form $s \rightarrow \mathbf{1} \oplus \cdots$ where no other $\mathbf{1}$ 's appear in $\cdots$.

For a more detailed discussion about stringlike excitations and their related membrane operators, see Ref. [37].

\section{Dimension reduction of generic topological orders}

We can reduce a $3+1 \mathrm{D}$ topological order $\mathcal{C}^{4}$ on spacetime $M^{3} \times S^{1}$ to $2+1 \mathrm{D}$ topological orders on spacetime $M^{3}$ by making the circle $S^{1}$ small [see Figs. 3 and 4(a)] $[42,43]$. In this limit, the $3+1 \mathrm{D}$ topological order $\mathcal{C}^{4}$ can be viewed as several $2+1 \mathrm{D}$ topological orders $\mathcal{C}_{i}^{3}$, $i=1,2, \ldots, N_{1}^{\mathrm{sec}}$, which happen to have degenerate ground-state energy. We denote such a dimensional reduction process by 


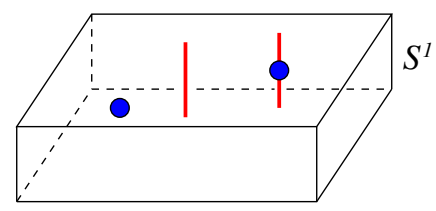

FIG. 3. The dimension reduction of $3 \mathrm{D}$ space $M^{2} \times S^{1}$ to $2 \mathrm{D}$ space $M^{2}$. The top and the bottom surfaces are identified, and the vertical direction is the compactified $S^{1}$ direction. A 3D pointlike excitation (the blue dot) becomes an anyon in 2D. A 3D stringlike excitation wrapping around $S^{1}$ (the red line) also becomes an anyon in 2D. The picture describes the untwisted sector where the $3 \mathrm{D}$ pointlike excitations have trivial holonomy as we move them around the compact direction $S^{1}$.

$$
\mathcal{C}^{4}=\bigoplus_{i=1}^{N_{1}^{\mathrm{sec}}} \mathcal{C}_{i}^{3},
$$

where $N_{1}^{\mathrm{sec}}$ is the number of sectors produced by the dimensional reduction.

We note that the different sectors come from the different holonomy of moving pointlike excitations around the $S^{1}$ (see Fig. 3). So the dimension reduction always contains a sector where the holonomy of moving any pointlike excitations around the $S^{1}$ is trivial. Such a sector is called the untwisted sector.

In the untwisted sector, there are three kinds of anyons. The first kind of anyons correspond to the $3+1 \mathrm{D}$ pointlike excitations. The second kind of anyons correspond to the $3+1 \mathrm{D}$ pure stringlike excitations wrapping around the compactified $S^{1}$. The third kind of anyons are bound states of the first two kinds [see Figs. 3 and 4(a)].

We point out that the untwisted sector in the dimension reduction can even be realized directly in $3 \mathrm{D}$ space without compactification. Consider a 2D submanifold in the 3D space (see Fig. 5), and put the 3D pointlike excitations on the $2 \mathrm{D}$ submanifold. We can have a loop of string across the 2D submanifold which can be viewed as an effective pointlike excitation on the $2 \mathrm{D}$ submanifold. We can also have a bound state of the above two types of effective

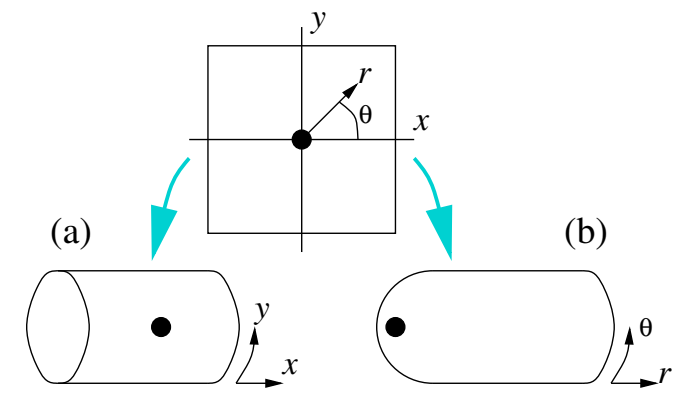

FIG. 4. Two ways to reduce a $2 \mathrm{D}$ space $M^{2}$ to $1 \mathrm{D}$ space. (a) The dimension reduction via compactifying the $y$ direction. (b) The dimension reduction via compactifying the $\theta$ direction. If we add the $z$ direction, the above become two ways to reduce a 3D space to $2 \mathrm{D}$ space.

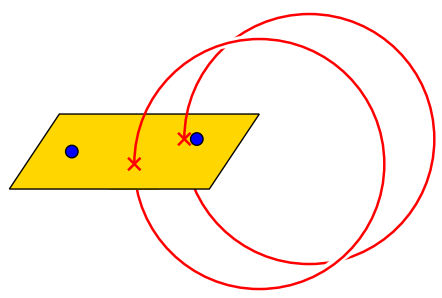

FIG. 5. The untwisted sector in the dimension reduction can be realized directly on a $2 \mathrm{D}$ submanifold in $3 \mathrm{D}$ space without compactification.

pointlike excitations on the $2 \mathrm{D}$ submanifold. Those effective pointlike excitations on the $2 \mathrm{D}$ submanifold can fuse and braid just like the anyons in $2+1 \mathrm{D}$. The principle of remote detectability requires those effective pointlike excitations to form a unitary modular tensor category. When we perform dimension reduction, the above unitary modular tensor category becomes the untwisted sector of the dimension-reduced $2+1 \mathrm{D}$ topological order.

Since the dimension-reduced $2+1 \mathrm{D}$ topological orders must be anomaly-free, they must be described by unitary modular tensor categories. Since the untwisted sector always contains $\operatorname{s} \mathcal{R e p}\left(G_{f}\right)$, we conclude that the untwisted sector of a dimension-reduced $3+1 \mathrm{D}$ EF topological order is a modular extension of $\operatorname{s} \mathcal{R e p}\left(G_{f}\right)$.

\section{Sectors of dimension reduction are 2+1D Drinfeld centers}

In Ref. [37], we show the following stronger result: Let the symmetric fusion category formed by the pointlike excitations be $\mathcal{E}, \mathcal{E}=\mathcal{R} \operatorname{ep}(G)$ or $\mathcal{E}=\operatorname{s} \mathcal{R e p}\left(G_{f}\right)$ for the $\mathrm{AB}$ or EF cases, respectively: The untwisted sector $\mathcal{C}_{\mathrm{untw}}^{3}$ of dimension reduction of a generic $3+1 \mathrm{D}$ topological orders must be the $2+1 \mathrm{D}$ topological order described by the Drinfeld center of $\mathcal{E}$ : $\mathcal{C}_{\text {untw }}^{3}=Z(\mathcal{E})$. Note that Drinfeld center $Z(\mathcal{E})$ is the minimal modular extension of $\mathcal{E}$. In the following, we generalize the above to any sector of dimension reduction.

First, let us recall the definition of a Drinfeld center. The Drinfeld center $Z(\mathcal{A})$ of a fusion category $\mathcal{A}$ is a braided fusion category, whose objects are pairs $\left(A, b_{A,-}\right)$, where $A$ is an object in $\mathcal{A}$ and $b_{A,-}$ is a set of isomorphisms $b_{A, X}: A \otimes X \cong X \otimes A, \quad \forall X \in \mathcal{A}$. The isomorphism $b_{A, X}$ is just the collection of unitary operators that connects the fusion spaces $\cdots \otimes A \otimes X \otimes \cdots$ and $\cdots \otimes X \otimes A \otimes \cdots$ for different backgrounds. They satisfy some selfconsistency conditions such as the hexagon equation:

$$
b_{A, Y} b_{A, X}=b_{A, X \otimes Y}
$$

where we omit the associativity constraints (or $F$ matrices) of $\mathcal{A}$ for simplicity (otherwise, there are in addition three $F$ matrices involved, in total six terms, hence the name hexagon). $b_{A, X}$ is called a half-braiding. 


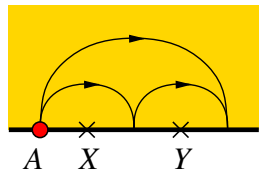

FIG. 6. If a (composite) boundary excitation can be lifted into the bulk, its half-braiding with other boundary excitations must satisfy some self-consistency conditions. The above illustrates the hexagon equation $b_{A, Y} b_{A, X}=b_{A, X \otimes Y}$.

Physically, we may view the objects in $\mathcal{A}$ as the pointlike topological excitations living on the boundary of a $2+1 \mathrm{D}$ topological order. In general, a boundary excitation trapped by a potential on the boundary cannot be lifted into the bulk. Physically, this means that as the trapping potential moves into the bulk, the ground-state subspace will be joined by some high-energy eigenstates to form a new ground-state subspace. But we may choose the boundary trapping potential very carefully, so that the ground-state subspace is formed by accidentally degenerate boundary excitations. In this case, we say that the excitation trapped by the boundary potential is a direct sum of those boundary excitations. Such an excitation corresponds to a composite object in the fusion category $\mathcal{A}$. Now the question is which composite object (or direct sum of boundary excitations) can be lifted into the bulk (i.e., the ground-state subspace rotates only by unitary transformation as we move the trapping potential into the bulk).

We try to answer this question by exchanging a composite object $A$ in $\mathcal{A}$ with an arbitrary boundary excitation $X$ and study the unitary transformation $b_{A, X}$ induced by such an exchange. If $A$ can be lifted into the bulk, this $b_{A, X}$ can be interpreted as coming from the half-braiding (see Fig. 6). There are self-consistency conditions from those half-braidings. If we find a composite object $A$ whose halfbraidings satisfy those consistent conditions, we believe that the object $A$ can be lifted into the bulk.

However, there is an additional subtlety: Even when we require that the ground-state subspace rotates only by unitary transformation as we move the trapping potential into the bulk, there are still different ways to move a composite boundary excitation $A$ into the bulk, which correspond to different pointlike excitations in the bulk. Those different bulk excitations can be distinguished by their different half-braiding properties with all the boundary excitations $X$. All the bulk excitations can be obtained this way. Therefore, the bulk excitations are given by pairs $\left(A, b_{A,-}\right)$, which correspond to the objects in the Drinfeld center $Z(\mathcal{A})$.

Mathematically, the morphisms of $Z(\mathcal{A})$ between the pairs $\left(A, b_{A,-}\right),\left(B, b_{B,-}\right)$ are a subset of morphisms between $A, B$, such that they commute with the halfbraidings $b_{A,-}, b_{B,-}$. Two pairs $\left(A, b_{A,-}\right),\left(B, b_{B,-}\right)$ are equivalent if there is an isomorphism in $Z(\mathcal{A})$ between them; namely, there is an isomorphism, a collection of unitary operators between the fusion spaces $\cdots \otimes A \otimes \cdots$,

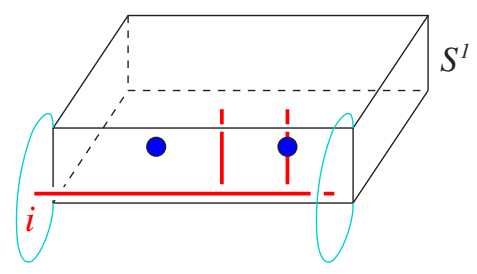

FIG. 7. The dimension reduction of $3 \mathrm{D}$ space $M^{2} \times S^{1}$ to $2 \mathrm{D}$ space $M^{2}$ for a twisted sector. The top and the bottom surfaces are identified, and the vertical direction is the compactified $S^{1}$ direction. The picture describes a twisted sector where the $3 \mathrm{D}$ pointlike excitations have nontrivial holonomy as we move them around the compact direction $S^{1}$. Such a nontrivial holonomy is represented by the red horizontal line (the base string) that characterizes this nontrivial holonomy, and hence the twisted sector. A $3 \mathrm{D}$ pointlike excitation not on the base string (the blue dot) becomes an anyon particle in 2D. A 3D stringlike excitation wrapping around the base string (the red line) also becomes an anyon particle in $2 \mathrm{D}$.

$\cdots \otimes B \otimes \cdots$, that commutes with the half-braidings $b_{A,-}$, $b_{B,-}$. The fusion and braiding of $\left(A, b_{A,-}\right)$ 's is given by

$$
\begin{aligned}
\left(A, b_{A,-}\right) \otimes\left(B, b_{B,-}\right) & =\left(A \otimes B,\left(b_{A,-} \otimes \mathrm{id}_{B}\right)\left(\mathrm{id}_{A} \otimes b_{B,-}\right)\right), \\
c_{\left(A, b_{A,-}\right),\left(B, b_{B,-}\right)} & =b_{A, B} .
\end{aligned}
$$

In other words, to half-braid $A \otimes B$ with $X$, one just half-braids $B$ and $A$ successively with $X$, and the braiding between $\left(A, b_{A,-}\right)$ and $\left(B, b_{B,-}\right)$ is nothing but the halfbraiding.

In a sector $\mathcal{C}_{i}^{3}$ of dimension reduction [see Fig. 4(a)], as we move the $3+1 \mathrm{D}$ pointlike excitation around the compactified $S^{1}$, we may obtain nontrivial holonomy. This nontrivial holonomy corresponds to those induced by moving the pointlike excitation around a $3+1 \mathrm{D}$ string of type $i$. Therefore, we may view a twisted sector $\mathcal{C}_{i}^{3}$ as having a base string $i$ going through the compactified $S^{1}$ (see Fig. 7). The particles in $\mathcal{C}_{i}^{3}$ come from the strings (including the trivial strings) wrapping around the compactified $S^{1}$. Thus, the particles in $\mathcal{C}_{i}^{3}$ come from the strings (including the trivial strings) wrapping around the base string $i$.

Naturally, the content of anyons in the twisted sector $\mathcal{C}_{i}^{3}$ depends on the twist (i.e., the base string $i$ ). To understand how $\mathcal{C}_{i}^{3}$ depends on the base string $i$, we note that, as we move a $3+1 \mathrm{D}$ particle to the base string $i$, the degeneracy of the fusion space may split. Thus, a $3+1 \mathrm{D}$ particle may behave like a direct sum of several particles on the base string $i$. Those particles on the base string are regarded as confined on the base string (see the particle $p$ in Fig. 8). Also, shrinking a string loop $s$ around the base string $i$ to a point on the base string may also correspond to a direct sum of several particles on the base string. We also regard those particles as confined on the base string (see the particle $p_{s}^{\text {shr }}$ in Fig. 8). 

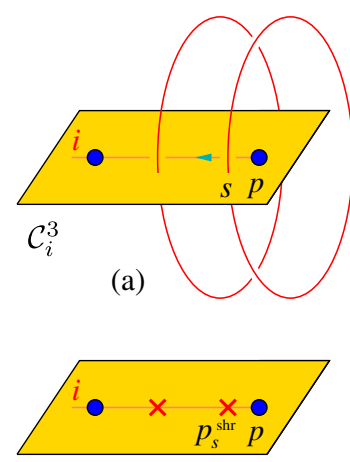

(c)

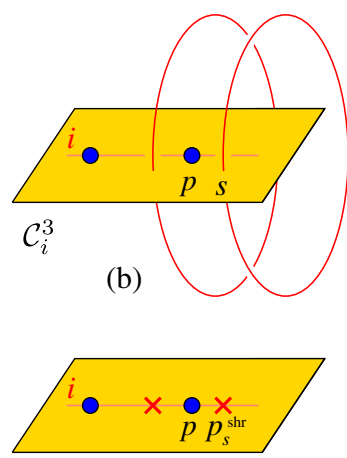

(d)
FIG. 8. From (a) to (b), the particle $p$ on the base string $i$ is moved through the loop $s$. (c),(d) are obtained from (a),(b) by shrinking loops. Shrinking thus induces a "half-braiding" isomorphism $c_{s, p}^{\text {shr }}$ from (c) to (d).

The pointlike excitations confined on the base string naturally form a fusion category, denoted by $\mathcal{A}_{i}$ [69]. In fact, in the second dimension-reduction scheme in Fig. 4(b), the base string at $(x, y)=(0,0)$ exactly behaves like a gapped boundary of the dimension-reduced theory, as shown in Fig. 4(b). The above discussions then immediately imply that $\mathcal{C}_{i}^{3}=Z\left(\mathcal{A}_{i}\right)$.

On the other hand, similar to the untwisted sector, $\mathcal{C}_{i}^{3}$ can also be effectively represented, in $3+1 \mathrm{D}$ without dimension reduction, by the three-loop braiding [40,41], as shown in Fig. 8, which is equivalent to Fig. 4(b) around the base string. As dimension reduction does not apply to general spacetime topology, next we use the three-loop braiding picture to prove the result in a more rigorous way, which is almost parallel to that in Ref. [37].

The important thing to note is that loops encircling the base string can shrink to the base string. When we shrink a loop $s$ (including bound states of particles with strings, in particular, pointlike excitations viewed as bound states with the trivial string) to the base string, it becomes a confined pointlike excitation $p_{s}^{\text {shr }}$ in $\mathcal{A}_{i}$ :

$s \rightarrow p_{s}^{\mathrm{shr}}=p_{1} \oplus p_{2} \oplus \cdots, \quad p_{1}, p_{2}, \ldots \in \mathcal{A}_{i}$.

So if we consider only fusion, the particles $s$ in the dimension-reduced sector $\mathcal{C}_{i}^{3}$ can all be viewed as the particles in $\mathcal{A}_{i}$, regardless if they come from the 3D particles or 3D strings. In general, simple bulk particles $p \in \mathcal{E}$ may become composite in $\mathcal{A}_{i}$, unless in the untwisted sector.

Now imagine we move a confined particle $p$ on the base string, through the loop $s$ encircling the base string. This movement induces an isomorphism between the initial and end states where the loop $s$ is shrunk (see Fig. 8):

$$
c_{s, p}^{\mathrm{shr}}: p_{s}^{\mathrm{shr}} \otimes p \cong p \otimes p_{s}^{\mathrm{shr}},
$$

which is automatically a half-braiding on the particle $p_{s}^{\text {shr }}$. Thus, $\left(p_{s}^{\mathrm{shr}}, c_{s,-}^{\mathrm{shr}}\right)$, by definition, is an object in the Drinfeld center $Z\left(\mathcal{A}_{i}\right)$. In $2+1 \mathrm{D}$ topological orders, when a boundary particle can be lifted into the bulk, it has halfbraidings with other boundary particles; here, the story is very similar-when a particle confined on the base string can either be lifted from the base string or expand to another loop excitation encircling the base string, it has half-braidings with other particles confined on the base string.

Shrinking thus induces a functor

$$
\begin{aligned}
\mathcal{C}_{i}^{3} & \rightarrow Z\left(\mathcal{A}_{i}\right), \\
s & \mapsto\left(p_{s}^{\mathrm{shr}}, c_{s,-}^{\mathrm{shr}}\right),
\end{aligned}
$$

which is obviously monoidal and braided, i.e., preserves fusion and braiding. As $\mathcal{C}_{i}^{3}$ is a unitary modular tensor category, this functor must be fully faithful [70], namely, an embedding functor; in other words, $\mathcal{C}_{i}^{3}$ can be viewed as a full subcategory of $Z\left(\mathcal{A}_{i}\right)$. However, if there is some $X \in$ $Z\left(\mathcal{A}_{i}\right)$ but $X \notin \mathcal{C}_{i}^{3}$ (namely, $X$ is not physically present), certain particles in $\mathcal{A}_{i}$ will be indistinguishable by threeloop braidings. Therefore, by the principle of remote detectability, we must have

$$
\mathcal{C}_{i}^{3}=Z\left(\mathcal{A}_{i}\right)
$$

In general, it is not easy to determine the confined particles $\mathcal{A}_{i}$ on the base string $i$. But, for the untwisted sector, the base string is trivial, and thus $\mathcal{A}_{\text {untw }}=\mathcal{E}$ and $\mathcal{C}_{\text {untw }}^{3}=Z(\mathcal{E})$. As $Z(\mathcal{E})$ is known well, many properties can be easily extracted. For example, objects in $Z\left[\operatorname{s} \mathcal{R e p}\left(G_{f}\right)\right]$ have the form $(\chi, \rho)$, where $\chi$ is a conjugacy class of $G_{f}$ and $\rho$ is a representation of the subgroup of $G_{f}$ that centralizes $\chi$. One then concludes

(1) a looplike excitation in a 3+1D topological order always has an integer quantum dimension, which is $|\chi| \operatorname{dim} \rho$;

(2) pure strings ( $\rho$ trivial) always correspond to conjugacy classes of the group.

For $3+1 \mathrm{D}$ EF topological orders, as the fermion number parity $z$ is in the center of $G_{f}$, its conjugacy class has only one element. We have the following corollary, which is used in later discussions:

Corollary.-In all 3 $+1 \mathrm{D}$ EF topological orders, there is an invertible pure $Z_{2}^{f}$ flux loop excitation, corresponding to the conjugacy class of fermion number parity $z$.

\section{E. Condensing all the bosonic pointlike excitations}

Starting from a $3+1 \mathrm{D} \mathrm{EF}$ topological order $\mathcal{C}_{\mathrm{EF}}^{4}$, we can condense all the bosonic pointlike excitations described by $\mathcal{R} \operatorname{ep}\left(G_{b}\right)$, to obtain a new $3+1 \mathrm{D}$ EF topological order $\tilde{\mathcal{C}}^{4}$. After $\operatorname{Rep}\left(G_{b}\right)$ is condensed, all bosonic pointlike 
excitations become the trivial pointlike excitation in $\tilde{\mathcal{C}}^{4}$, while all fermionic pointlike excitations become the same fermionic pointlike excitations with quantum dimension 1 . In other words, the pointlike excitations in the new topological order $\tilde{\mathcal{C}}^{4}$ are described by $\operatorname{s} \mathcal{R e p}\left(Z_{2}^{f}\right)$.

Let us describe the boson condensation in more detail. The pointlike excitations described by $\operatorname{s} \mathcal{R} \operatorname{ep}\left(G_{f}\right)$ satisfy a fusion rule described by the fusion of the representations of $G_{f}$. The corresponding conservation is a conservation of $G_{f}$ quantum numbers (i.e., representations). Therefore, there is an emergent $G_{f}$ symmetry in the topological order $\mathcal{C}_{\mathrm{EF}}^{4}$. We note that a $G_{b}$-valued bosonic field forms a reducible representation of $G_{b}$ that includes all the irreducible representations. Thus, the condensation of such a bosonic field corresponds to the condensation of all the bosonic pointlike excitations. This condensation breaks the emergent $G_{f}$ symmetry in $\mathcal{C}_{\mathrm{EF}}^{4}$ to the emergent $Z_{2}^{f}$ symmetry in $\tilde{\mathcal{C}}^{4}$.

This picture of $\tilde{\mathcal{C}}^{4}$ seems to suggests a $\left|G_{b}\right|$-fold groundstate degeneracy due to the spontaneous symmetry breaking. However, since the $G_{f}$ symmetry is emergent, at a finite length scale $l$, the $G_{f}$ symmetry is broken by an amount of the order of $e^{-l / \xi}$, where $\xi$ is the correlation length of local orders in $\mathcal{C}_{\mathrm{EF}}^{4}$. So $\left|G_{b}\right|$-fold ground-state degeneracy is split by an amount of the order of $e^{-l / \xi} \Delta$, where $l$ is the interparticle separation for the condensed bosons and $\Delta$ is the gap of $\mathcal{C}_{\mathrm{EF}}^{4}$. Therefore, $\tilde{\mathcal{C}}^{4}$ does not have ground-state degeneracy coming from spontaneous symmetry breaking, since the splitting $e^{-l / \xi} \Delta$ is finite in the thermodynamic limit.

What are the stringlike excitations in $\tilde{\mathcal{C}}^{4}$ ? Although the pointlike excitations in $\tilde{\mathcal{C}}^{4}$ are very simple and can detect only simple strings, the stringlike excitations can braid among themselves and detect each other. Thus, $\tilde{\mathcal{C}}^{4}$ might contain complicated stringlike excitations.

However, as pointed out in Ref. [37], to remotely detect an unlinked string loop $s$ in the space $S^{3}$, we need to use another string loop $s^{\prime}$ and the two-loop braiding between $s$ and $s^{\prime}$ to remotely detect $s$. However, on $S^{3}$ such a two-loop braiding corresponds to braiding a particle $p^{\prime}$ around the string loop $s$, where the particle $p^{\prime}$ is the shrinking of the string loop $s^{\prime}$. Since, for $\tilde{\mathcal{C}}^{4}, p^{\prime}$ can be only the trivial particle or the fermion $f$, the strings that they can distinguish are also very simple. In other words, there should be only one type of simple pure string $s_{1}$, which has nontrivial braiding with $f$ (i.e., behaves like a $\pi$ flux for the fermion $f$ ).

This result can also be obtained using the dimension reduction discussed above, plus the following conjecture:

Conjecture.-There is a one-to-one correspondence between the simple types of stringlike excitations in $3+1 \mathrm{D}$ and the simple types of pointlike excitations in the $2+1 \mathrm{D}$ untwisted sector of the dimension reduction.
To understand the above conjecture, we note that each simple string in $3+1 \mathrm{D}$ reduces to a particle in $2+1 \mathrm{D}$ in the untwisted sector (see Fig. 3). However, the 2+1D particle might be composite (i.e., a direct sum of several simple types of particles in $2+1 \mathrm{D})$. But since the simple strings in $3+1 \mathrm{D}$ are stable against any nonlocal perturbations around the strings, the corresponding particles in $2+1 \mathrm{D}$ are stable against any local perturbations around the particles. Thus, the corresponding $2+1 \mathrm{D}$ particles are actually simple types. This reasoning leads to the above conjecture.

With such a conjecture, the $3+1 \mathrm{D}$ stringlike excitations are determined by the $2+1 \mathrm{D}$ pointlike excitations described by $\mathcal{E}=\operatorname{s} \mathcal{R} \operatorname{ep}\left(Z_{2}^{f}\right)$. In particular, the untwisted sector of the dimension reduction must be the Drinfeld center $Z(\mathcal{E})=Z\left[\operatorname{s} \mathcal{R e p}\left(Z_{2}^{f}\right)\right]$, which is nothing but the $2+1 \mathrm{D}$ $Z_{2}$-gauge theory. There are only four types of $2+1 \mathrm{D}$ anyons: Two of them correspond to the $3+1 \mathrm{D}$ pointlike excitations in $\operatorname{s} \mathcal{R e p}\left(Z_{2}^{f}\right)$, and the other two correspond to the $3+1 \mathrm{D}$ stringlike excitations. The fusion rule between the four anyons in the $2+1 \mathrm{D} Z_{2}$-gauge theory is described by the $Z_{2} \times Z_{2}$ group, which leads to the fusion rule between the loops and the fermion $f$ :

$$
\begin{aligned}
f \otimes f & =\mathbf{1}, \quad f \otimes s_{1}=s_{2}, \quad f \otimes s_{2}=s_{1}, \\
s_{1} \otimes s_{1} & =s_{2} \otimes s_{2}=\mathbf{1}, \quad s_{1} \otimes s_{2}=f .
\end{aligned}
$$

The above also implies the shrinking rule for the loops to be

$$
s_{1} \rightarrow \mathbf{1}, \quad s_{2} \rightarrow f .
$$

We also find that the braiding phases between the fermion $f$ and the two loops $s_{i}$ are given by -1 , and the braiding phase between two $s_{1}$ or two $s_{2}$ 's is 1 . The braiding phase between $s_{1}$ and $s_{2}$ is -1 . Here, the invertible loop $s_{1}$ is the just the $Z_{2}^{f}$ flux loop $z$.

We see that $\tilde{\mathcal{C}}^{4}$ contains only one type of pure simple string $s_{1}$ which shrinks to a single $\mathbf{1}$. The other loop $s_{2}$ is the bound state of $s_{1}$ and the fermion $f$. The loop $s_{1}$ has a trivial two-loop braiding with itself.

We further investigate the braiding properties of $s_{1}$ by studying the twisted sector of dimension reduction with base string $s_{1}$. What are the confined pointlike excitations $\mathcal{A}_{s_{1}}$ on $s_{1}$ ? There is at least a $Z_{2}$ excitation in $\mathcal{A}_{s_{1}}$ coming from the bulk fermion. On the other hand, there should be at most four different types of particles in $\mathcal{C}_{s_{1}}^{3}$, corresponding to $\mathbf{1}, f, s_{1}$, and $s_{2}$ encircling the base string. There is only one unitary modular tensor category, which is a Drinfeld center, satisfying the above constraints; it is again $\mathcal{C}_{s_{1}}^{3}=$ $Z\left[\operatorname{sinep}\left(Z_{2}^{f}\right)\right]$ and $\mathcal{A}_{s_{1}}=\operatorname{s} \mathcal{R} \operatorname{ep}\left(Z_{2}^{f}\right)$. Thus $s_{1}$, $s_{2}$ encircling $s_{1}$ correspond to $Z_{2}$ bosons in $Z\left[\operatorname{sinep}\left(Z_{2}^{f}\right)\right]$, which means that the three-loop braiding of $s_{1}$ strings is also trivial. 
Another tricky point is that the trivial string 1, although with totally trivial braiding properties, may still secretly be a pSC chain. Thus, one may wonder if $s_{1}$ has any nontrivial interplay with pSC chains; for example, $s_{1} \otimes s_{1}=\mathbf{1}$ is, in fact, a pSC chain. But note that, when a pSC chain is linked with a $Z_{2}^{f}$ flux loop (namely, $s_{1}$ ), the fermion number parity will be changed. Thus, if $s_{1} \otimes s_{1}$ is a pSC chain, when encircling the base string $s_{1}$ (namely, in $\mathcal{C}_{s_{1}}^{3}$ ), one should have $s_{1} \otimes s_{1}=f$. The fact that $\mathcal{C}_{s_{1}}^{3}=Z\left[\operatorname{s} \mathcal{R} \operatorname{ep}\left(Z_{2}^{f}\right)\right]$ excludes such a possibility, since, for any particle $a$ in $\mathcal{C}_{s_{1}}^{3}=Z\left[\operatorname{s} \operatorname{Rep}\left(Z_{2}^{f}\right)\right], a \otimes a$ is always a boson. For details, see Sec. VIII C.

We thus conjecture that the pure string $s_{1}$ has totally trivial braiding properties and it can be condensed to obtain another topological order $\mathcal{D}^{4}$, which turns out to be trivial. To see this, note that condensing the pure string $s_{1}$ corresponds to condensing the corresponding topological boson in the untwisted sector described by $2+1 \mathrm{D} Z_{2}$-gauge theory $Z\left[\operatorname{s} \mathcal{R} \operatorname{ep}\left(Z_{2}^{f}\right)\right]$, which changes the untwisted sector to a trivial phase. So the untwisted sector of dimension-reduced $\mathcal{D}^{4}$ is trivial, which implies that $\mathcal{D}^{4}$ has no nontrivial pointlike and stringlike excitations.

We can also obtain such a result by noticing that, in $\mathcal{D}^{4}$, the fermions and $s_{2}$ are confined, due to the nontrivial braiding with $s_{1}$, and $s_{1}$ becomes the ground state (i.e., condensed). Thus, $\mathcal{D}^{4}$ has no nontrivial bulk excitations and must be an invertible topological order. But in $3+1 \mathrm{D}$, all invertible topological orders are trivial $[52,71,72]$. Thus, $\mathcal{D}^{4}$ is a trivial phase, which means that we can create a gapped boundary of $\tilde{\mathcal{C}}^{4}$ by condensing $s_{1}$ strings. Such a boundary contains only one fermionic particle $f$ with a $Z_{2}$ fusion rule:

$$
f \otimes f=\mathbf{1} .
$$

For any $\tilde{\mathcal{C}}^{4}$, condensing strings lead to the same above boundary, on which there is no nontrivial string excitation, and the only nontrivial pointlike excitation is the fermion. Using the principle that the boundary uniquely determines the bulk $[52,54,55]$, we conclude that all the $\tilde{\mathcal{C}}^{4}$ 's that satisfy the above properties are actually the same topological order, which is called the $Z_{2}^{f}$ topological order, denoted by $\mathcal{C}_{Z_{2}^{f}}^{4}$ : In any $3+1 \mathrm{D}$ EF topological order $\mathcal{C}_{\mathrm{EF}}^{4}$, condensing all the bosonic pointlike excitations in $\operatorname{Rep}\left(G_{b}\right)$ produces the same unique $3+1 \mathrm{D}$ topological order $\mathcal{C}_{Z_{2}^{f}}^{4}$.

The topological order $\mathcal{C}_{Z_{2}^{f}}^{4}$ is constructed on a cubic lattice [73]. It is also called twisted $Z_{2}$-gauge theory where the $Z_{2}$ charge is fermionic and is realized by the $3+1 \mathrm{D}$ Levin-Wen string-net model [38]. $\mathcal{C}_{Z_{2}^{f}}^{4}$ can also be realized by the Walker-Wang model [74] or by a 2-cocycle lattice theory [75]. In this paper, we refer to $\mathcal{C}_{Z_{2}^{f}}^{4}$ as the $Z_{2}^{f}$ topological order.

\section{ALL 3 + 1D BOSONIC TOPOLOGICAL ORDERS HAVE A GAPPABLE BOUNDARY}

It is well known that $2+1 \mathrm{D}$ topological orders with a nonzero chiral central charge $c$ cannot have a gapped boundary, which can be understood from the induced gravitational Chern-Simons term in the effective action for such a kind of topological orders. Since there is no gravitational Chern-Simons term in $3+1 \mathrm{D}$, this might suggest that all $3+1 \mathrm{D}$ bosonic topological orders have a gappable boundary. However, such a reasoning is not correct. In fact, there are $2+1 \mathrm{D}$ topological orders with a zero chiral central charge (i.e., with no gravitational Chern-Simons term) that cannot have a gapped boundary [51].

For a $2+1 \mathrm{D}$ topological order described by a unitary modular tensor category $\mathcal{C}^{3}$, if $\mathcal{C}^{3}$ has a condensable algebra [76], then we can condense the bosons in the condensable algebra to obtain another $2+1 \mathrm{D}$ topological order described by a different unitary modular tensor category $\mathcal{D}^{3}$. Now we ask, is there a gapped domain wall between the two topological orders $\mathcal{C}^{3}$ and $\mathcal{D}^{3}$ ? In fact, we can show that there exists a $1+1 \mathrm{D}$ anomalous topological order, described by unitary fusion category $\mathcal{A}_{w}^{2}$, which is mathematically the category of modules over the condensable algebra, such that the Drinfeld center of $\mathcal{A}_{w}^{2}$ is $\mathcal{C}^{3} \otimes \overline{\mathcal{D}}^{3}$. Here, $\mathcal{C}^{3} \otimes \overline{\mathcal{D}}^{3}$ is the $2+1 \mathrm{D}$ topological order formed by stacking two topological orders, $\mathcal{C}^{3}$ and $\overline{\mathcal{D}}^{3}$, where $\overline{\mathcal{D}}^{3}$ is the time-reversal conjugate of $\mathcal{D}^{3}$. This means that it is consistent to view $\mathcal{A}_{w}^{2}$ as the domain wall between $\mathcal{C}^{3}$ and $\mathcal{D}^{3}$. Then, we conjecture that there exists a gapped domain wall between $\mathcal{C}^{3}$ and $\mathcal{D}^{3}$ that is described by $\mathcal{A}_{w}^{2}$.

In the last section, we have seen that condensing all the bosonic excitations described by $\operatorname{Rep}\left(G_{b}\right)$ in a $3+1 \mathrm{D}$ EF topological order $\mathcal{C}_{\mathrm{EF}}^{4}$ give us an unique $3+1 \mathrm{D}$ topological order $\mathcal{C}_{Z_{2}^{f}}^{4}$. This result can also be obtained by noticing that the condensation of $\mathcal{R e p}\left(G_{b}\right)$ is described by a condensable algebra, and there is only one condensable algebra if we want to condense all $\mathcal{R e p}\left(G_{b}\right)$. So there is only one way to condense all $\mathcal{R e p}\left(G_{b}\right)$ which produces a unique state $\mathcal{C}_{Z_{2}^{f}}^{4}$.

We expect that, similar to the $2+1 \mathrm{D}$ case, such an unique condensation also produces an unique unitary fusion 2-category $\mathcal{A}_{w}^{3}$, such that the generalized Drinfeld center, or bulk center, of $\mathcal{A}_{w}^{3}$ is $\mathcal{C}_{\mathrm{EF}}^{4} \otimes \overline{\mathcal{C}}_{Z_{2}^{f}}^{4}$, and we can view $\mathcal{A}_{w}^{3}$ as the canonical domain wall between $\mathcal{C}_{\mathrm{EF}}^{4}$ and $\mathcal{C}_{Z_{2}^{f}}^{4}$. This result motivates us to conjecture that there exists a gapped domain wall between two $3+1 \mathrm{D}$ EF topological orders $\mathcal{C}_{\mathrm{EF}}^{4}$ and $\mathcal{C}_{Z_{2}^{f}}^{4}$.

There is a heuristic physical argument to support the above conjecture. The particles in the condensable algebra are all bosons which form a symmetric fusion category $\mathcal{R e p}\left(G_{b}\right)$. Those bosons have an emergent symmetry 
described by $G_{b}$. As discussed in Sec. III E, $\mathcal{C}_{Z_{2}^{f}}^{4}$ can be viewed as the boson condensed state with a small symmetry-breaking perturbation. Such a kind of state is well studied in physics which always allows a gapped domain wall between itself and its uncondensed parent state. Therefore, the domain wall between two $3+1 \mathrm{D} \mathrm{EF}$ topological orders $\mathcal{C}_{\mathrm{EF}}^{4}$ and $\mathcal{C}_{Z_{2}^{f}}^{4}$ can always be gapped. In Sec. III E, we show that $\mathcal{C}_{Z_{2}^{f}}^{4}$ topological order can have a gapped boundary, which allows us to argue that all $3+1 \mathrm{D}$ EF topological orders have a gappable boundary.

Using a similar argument, we can argue that all $3+1 \mathrm{D}$ $\mathrm{AB}$ topological orders have a gappable boundary. In fact, the argument is simpler for the $\mathrm{AB}$ case, since boson condensation directly results in a trivial phase, with no intermediate $\mathcal{C}_{Z_{2}^{f}}^{4}$ topological order. Hence, all $3+1 \mathrm{D}$ bosonic topological orders have a gappable boundary.

\section{UNIQUE CANONICAL DOMAIN WALLS BETWEEN 3 + 1D EF TOPOLOGICAL ORDERS AND $Z_{2}^{f}$ TOPOLOGICAL ORDER $\mathcal{C}_{Z_{2}^{f}}^{4}$}

In this section, we derive the properties of the fusion 2-category $\mathcal{A}_{w}^{3}$ which describes the domain wall between $\mathcal{C}_{\mathrm{EF}}^{4}$ and $\mathcal{C}_{Z_{2}^{f}}^{4}$.

\section{A. All simple boundary strings and boundary particles have quantum dimension 1}

After condensing all bosonic particles $\mathcal{R e p}\left(G_{b}\right)$, the only nontrivial particle on the canonical domain wall is the fermion $f$ with quantum dimension 1 . Such a fermion can be lifted into one side of the domain wall with the $Z_{2}^{f}$ topological order $\mathcal{C}_{Z_{2}^{f}}^{4}$. On the other side of the domain wall with $3+1 \mathrm{D}$ EF topological order $\mathcal{C}_{\mathrm{EF}}^{4}$, if we bring the fermions in $\operatorname{s} \operatorname{Rep}\left(G_{f}\right)$ to the boundary, it will become a direct sum (i.e., accidental degenerate copies) of several $f$ 's.

What are the stringlike excitations on the domain wall? On the $\mathcal{C}_{Z_{2}^{f}}^{4}$ side of the domain wall, there is only one type of pure simple stringlike excitation-the $Z_{2}^{f}$ flux loop with quantum dimension 1 . Bringing such a string to the domain wall gives us a $Z_{2}^{f}$ flux loop on the wall. We can also bring strings in $\mathcal{C}_{\mathrm{EF}}^{4}$ to the domain wall. In general, a string in $\mathcal{C}_{\mathrm{EF}}^{4}$ becomes a direct sum of simple boundary strings.

Let us focus on the simple loop excitations on the canonical domain wall. A loop excitation shrunk to a point may become a direct sum of pointlike excitations [see Eq. (7)]:

$$
s \rightarrow n \mathbf{1} \oplus m f,
$$

where $\mathbf{1}$ and $f$ are the trivial and fermionic pointlike excitations, respectively. When $n=0$, the string is not pure. Another possibility is that $n>1$. In this case, the string is not simple. When $m>1$, the string is also not simple, since, when $s$ fuses with an invertible fermion, its shrinking rule becomes

$$
s \otimes f \rightarrow m \mathbf{1} \oplus n f,
$$

which is not simple. Supposing $s \otimes f=s_{1} \oplus s_{2} \oplus \cdots$, then $s=s \otimes f \otimes f=\left(s_{1} \otimes f\right) \oplus\left(s_{2} \otimes f\right) \oplus \cdots$ is not simple. Therefore, simple loop excitations on the domain wall have three possible shrinking rules:

$$
s_{b} \rightarrow \mathbf{1}, \quad s_{f} \rightarrow f, \quad s_{K} \rightarrow \mathbf{1} \oplus f .
$$

In the following, we show, by contradiction, that a simple string like $s_{K}$ with quantum dimension 2 cannot exist on the domain wall.

First, the invertible $Z_{2}^{f}$ flux loop $z$ exists in both sides, $\mathcal{C}_{\mathrm{EF}}^{4}$ and $\mathcal{C}_{Z_{2}^{f}}^{4}$, of the domain wall. We are able to braid $z$ around the domain-wall excitations. As $z$ is invertible, such braiding leads to only a $U(1)$ phase factor, denoted by $\theta(z,-)$. In particular, $\theta(z, f)=-1$, which is the defining property of $Z_{2}^{f}$ flux.

Second, fusing a fermion $f$ to a string $s_{K}$ which shrinks to $1 \oplus f$ will not change the string, namely, $s_{K} \otimes f=s_{K}$. Thus,

$\theta\left(z, s_{K}\right)=\theta\left(z, s_{K} \otimes f\right)=\theta\left(z, s_{K}\right) \theta(z, f)=-\theta\left(z, s_{K}\right)$,

which is contradictory. Physically, we can use the braiding of $z$ to detect the fermion number parity on the domain wall, which implies that excitations without a fixed fermion number parity, such as $s_{k} \rightarrow 1 \oplus f$, cannot be stable on the domain wall. Therefore, there is no simple domain-wall string with quantum dimension 2.

Thus, a simple loop on the boundary shrinks to a unique particle, $\mathbf{1}$ or $f$, with quantum dimension 1. A simple pure loop on the boundary always shrinks to a single $\mathbf{1}$. This is an essential property in the following discussions: All simple pure loops on the domain wall have a quantum dimension $d=1$, and their fusion is grouplike. As the nonpure simple loops are all bound states of $f$ with pure simple loops, we consider only the simple pure loops. For the moment, we denote the group formed by the simple pure loops on the domain wall under fusion (see Fig. 11) by $H$.

\section{B. Fusion of domain-wall strings recovers the group}

The argument in this subsection is almost parallel to those in the AB case described in Ref. [37]. There are only a few modifications to address the fermionic nature. But to be self-contained we include a full argument here. It is 


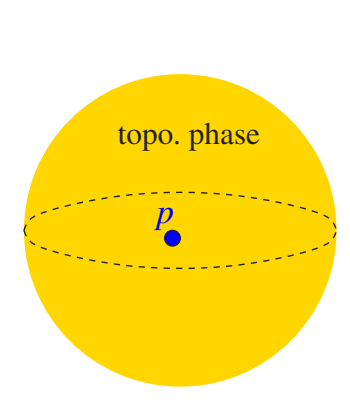

(a)

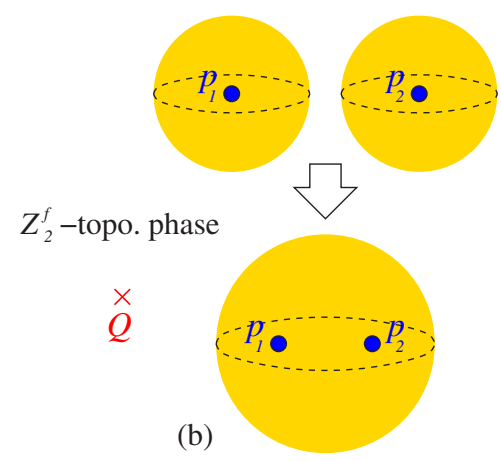

(b)

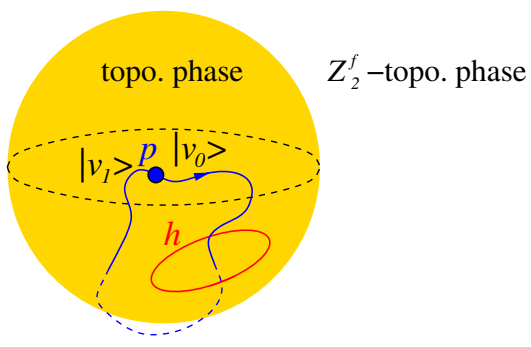

FIG. 10. Moving a particle (blue) around a loop excitation (red) on the domain wall. The solid line is in the $\mathcal{C}_{\mathrm{EF}}^{4}$ phase. The dashed line is in the $\mathcal{C}_{Z_{2}^{f}}^{4}$ phase.

FIG. 9. (a) The fusion space $F(p)$ for a 3-disk $D^{3}$ containing only one particle $p$. (b) $F$ preserves fusion of particles, $F\left(p_{1}\right) \otimes_{\mathbb{C}} F\left(p_{2}\right) \cong F\left(p_{1} \otimes p_{2}\right)$.

recommended that readers not familiar with Tannaka duality go through Appendix A first.

To apply the Tannaka duality, we need a physical realization of the super-fiber-functor. Consider a simple topology for the domain wall: Put the $3+1 \mathrm{D}$ topological order $\mathcal{C}_{\mathrm{EF}}^{4}$ in a 3-disk $D^{3}$ and the domain wall on $\partial D^{3}=S^{2}$, and outside is the condensed phase $\mathcal{C}_{Z_{2}^{f}}^{4}$. When there is only a particle $p$ in the 3-disk, a background particle $Q=1 \oplus f$ in the condensed phase $\mathcal{C}_{Z_{2}^{f}}^{4}$ [77], with no string and no other particles, we associate the corresponding fusion space to the particle $p$ and denote this fusion space by $F(p)$ (see Fig. 9). Viewed from very far away, a 3-disk containing a particle $p$ is like a particle in the condensed phase $\mathcal{C}_{Z_{2}^{f}}^{4}$, which has pointlike excitations $\mathrm{s} \mathcal{V} e \mathrm{~s}=\operatorname{s} \operatorname{Rep}\left(Z_{2}^{f}\right)$. In other words, $F(p)$ is like a local super-vector-space in sVec describing a 3-disk containing $p$. Alternatively, as discussed in Appendix A 5, if $p$ is moved out of the 3-disk through the domain wall and becomes a particle $p^{\prime}$ in the condensed phase, such $p^{\prime}$ is also the super-vector-space $F(p)$. When there are two 3-disks, each containing only one particle, $p_{1}$ and $p_{2}$, respectively, the fusion space is $F\left(p_{1}\right) \otimes_{\mathbb{C}} F\left(p_{2}\right)$. It is proved in Appendix A 5 that $F$ preserves fusion, $F\left(p_{1}\right) \otimes_{\mathbb{C}} F\left(p_{2}\right) \cong F\left(p_{1} \otimes p_{2}\right)$, and also preserves the braiding of particles. In other words, the assignment $p \rightarrow F(p)$ gives rise to a super-fiberfunctor. By the Tannaka duality, we can reconstruct a group $G_{f} \equiv \operatorname{Aut}(F)$, such that the particles in the bulk $\mathcal{C}_{\mathrm{EF}}^{4}$ are identified with $\mathrm{s} \mathcal{R e p}\left(G_{f}\right)$. Our goal is to show that the fusion group $H$ of the simple loops on the domain wall is the same as $G_{f}$.

To achieve this goal, we consider the process of adiabatically moving the trap that hold a particle $p$ along a loop (Fig. 10). Half of the loop is in the original topological state, and the other half is in the $Z_{2}^{f}$ topological state. When the trap is in the original topological state, there is a $d_{p}$-fold ground-state degeneracy. Here, we design the trap very carefully such that, along all the loop, the trap always has a clear gap above the $d_{p}$-fold degenerate ground states. This design means that the trap has $d_{p}$-fold accidental degeneracy when the trap is in the $Z_{2}^{f}$ topological ordered state. We also fine-tune the trap such that the nonAbelian geometric phase induced by adiabatically moving along the loop is the identity when no extra excitation is present.

Now we create a loop of pure simple string $h \in H$ on the domain wall, linked with the loop path of $p$ above, as shown in Fig. 10. As the pure simple string is invertible, inserting them will not change the ground-state degeneracy. However, moving the trap along the above path may change an initial state $\left|v_{0}\right\rangle \in F(p)$ into a different end state $\left|v_{1}\right\rangle \in F(p)$, after the particle moves around the string $h$ (see Fig. 10). Thus, braiding $p$ around $h$ induces a unitary map on the fusion space $F(p), \alpha_{p, h}:\left|v_{0}\right\rangle \mapsto\left|v_{1}\right\rangle$. bulk. If we braid them together (fusing them to one particle $\left.p_{1} \otimes p_{2}\right)$ around the simple loop $h$, we obtain the linear map $\alpha_{p_{1} \otimes p_{2}, h}$. If the fusion of the bulk particles is given by $p_{1} \otimes p_{2}=\bigoplus_{i} W_{i}$, we can split $p_{1} \otimes p_{2}$ to the irreducible representations $W_{i}$ and braid $W_{i}$ with $h$. It is easy to see the $\alpha_{p, h}$ maps are automatically compatible with such splitting; in other words, $\alpha_{p_{1} \otimes p_{2}, h}=\bigoplus_{i} \alpha_{W_{i}, h}$.

But it is also equivalent if we braid $p_{1}, p_{2}$ one after the other. But this braiding induces a unitary map on $F\left(p_{1} \otimes p_{2}\right)$. We would like to spatially separate $F\left(p_{1}\right)$ and $F\left(p_{2}\right)$. As discussed in Appendix A 5, moving either $p_{1}$ or $p_{2}$ out of the 3-disk leads to fusion space $F\left(p_{1}\right) \otimes_{\mathbb{C}} F\left(p_{2}\right)$. Thus, besides braiding $p_{1}, p_{2}$ with $h$ one after the other, we add several steps to move $p_{2}, p_{1}$ back and forth.

First, we move $p_{2}$ out of the 3-disk. Then, braiding $p_{1}$ alone with the loop excitation $h$ corresponds to the linear map $\alpha_{p_{1}, h} \otimes_{\mathbb{C}} \operatorname{id}_{F\left(p_{2}\right)}$. Next, we bring $p_{2}$ back into the 3-disk, following the same path when it was moved out so that $p_{2}$ does not braid with $h$ in this step.

Second, we move $p_{1}$ out of the 3-disk. Then, braiding $p_{2}$ alone with $h$ corresponds to $\operatorname{id}_{F\left(p_{1}\right)} \otimes_{\mathbb{C}} \alpha_{p_{2}, h}$. Next, again, we bring $p_{1}$ back, following the same path when it was moved out.
Next, consider that we have two particles $p_{1}, p_{2}$ in the 


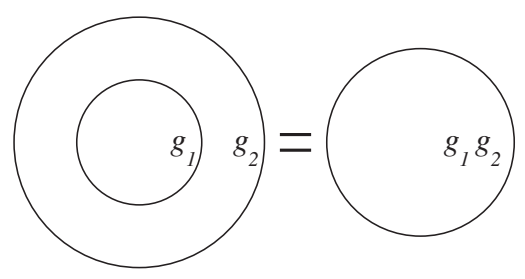

FIG. 11. The fusion of domain-wall stringlike excitations $s_{g_{1}}^{\text {bdry }} \otimes s_{g_{2}}^{\text {bdry }}=s_{g_{1} g_{2}}^{\text {bdry }}$, which can be abbreviated as $g_{1} \otimes g_{2}=g_{1} g_{2}$.

The steps of moving $p_{1}, p_{2}$ back and forth cancel, and only the braidings are left over. Therefore, in total we have the linear map $\alpha_{p_{1}, h} \otimes_{\mathbb{C}} \alpha_{p_{2}, h}$, which is equivalent to that induced by braiding $p_{1}, p_{2}$ together, $\alpha_{p_{1}, h} \otimes_{\mathbb{C}} \alpha_{p_{2}, h}=$ $\alpha_{p_{1} \otimes p_{2}, h}$. Using only irreducible representations,

$$
\alpha_{p_{1}, h} \otimes_{\mathbb{C}} \alpha_{p_{2}, h}=\bigoplus_{i} \alpha_{W_{i}, h}
$$

We show that these linear maps are compatible with the fusion of bulk particles.

Moreover, the pure simple loop $h$ provides such a unitary map $\alpha_{p, h}$ for each particle $p \in \operatorname{s} \mathcal{R} \operatorname{ep}\left(G_{f}\right)$ in $\mathcal{C}_{\mathrm{EF}}^{4}$; thus, the set of linear maps $\varphi(h) \equiv\left\{\alpha_{p, h}\right\}$ is an automorphism of the super-fiber-functor, $\varphi(h) \in G_{f} \equiv \operatorname{Aut}(F)$. In other words, we obtain a map $\varphi$ from the pure simple loops $H$ to $G_{f}$, $\varphi: H \rightarrow G_{f}$. It is also compatible with the fusion of simple loops on the domain wall. To see this compatibility, note that the path of braiding around two concentric simple loops $g_{1}, g_{2}$ (as in Fig. 11) separately can be continuously deformed to the braiding path around the two loops together or around their fusion $g_{1} \otimes g_{2}=g_{1} g_{2}$, which implies that $\varphi\left(g_{1}\right) \varphi\left(g_{2}\right)=\varphi\left(g_{1} g_{2}\right)$; namely, $\varphi$ is a group homomorphism.

Next, we show that $\varphi$ is, in fact, an isomorphism and $H=G_{f}$, which is a consequence of the following principles:

(1) If an excitation has trivial braiding with the condensed excitations, it must survive as a deconfined excitation in the condensed phase.

(2) There is no nontrivial bulk particle that has trivial half-braiding with all the domain-wall strings.

(1) is a general principle for condensations, while (2) is a remote detectability condition. By the folding trick, we can regard the domain wall as a boundary of the phase $\mathcal{C}_{\mathrm{EF}}^{4} \otimes \mathcal{C}_{Z_{2}^{f}}^{4}$. So we have similar remote detectability condition (2) near the domain wall as that near a boundary [37].

A typical half-braiding path is shown in Fig. 10, in the sense that half is in $\mathcal{C}_{\mathrm{EF}}^{4}$ and half in $\mathcal{C}_{Z_{2}^{f}}^{4}$. If $\alpha_{p, h}$ is the identity map, it implies trivial half-braiding between the particle $p$ in $\mathcal{C}_{\mathrm{EF}}^{4}$ and simple loop $h$ on the domain wall.

Now, we are ready to show that $\varphi: H \rightarrow G_{f}$ is an isomorphism:

(1) $\varphi$ is injective.-First, the $Z_{2}^{f}$ flux loop, denoted by $z$, which is simple, pure, and invertible and survives in the condensed phase $\mathcal{C}_{Z_{2}^{f}}^{4}$, must also be a pure simple loop on the domain wall. Namely, $Z_{2}^{f} \subset H$.

Consider $\operatorname{ker} \varphi$, namely, the pure simple loops that induce just identity linear maps on all particles in $\mathcal{C}_{\mathrm{EF}}^{4}$. On one hand, simple loops in $\operatorname{ker} \varphi$ have trivial half-braiding with all particles in $\mathcal{C}_{\mathrm{EF}}^{4}$. So they also have trivial braiding with the condensed excitations, namely, all the bosons in $\mathcal{C}_{\mathrm{EF}}^{4}$. By (1), they should all survive the condensation; in other words, $\operatorname{ker} \varphi$ is at most a subset of pure string excitations in $\mathcal{C}_{Z_{2}^{f}}^{4}$, $\operatorname{ker} \varphi \subset Z_{2}^{f}$. On the other hand, the linear map $\alpha_{p, z}$ induced by the $Z_{2}^{f}$ flux loop $z$ is not the identity map on fermions, so $z \notin \operatorname{ker} \varphi$.

Therefore, we see that $\operatorname{ker} \varphi$ must be trivial, which means $\varphi$ is injective.

(2) $\varphi$ is surjective.-We already showed that $\varphi: H \rightarrow$ $G_{f}$ is injective, so we can view $H$ as a subgroup of $G_{f}$.

Now consider a special particle in $\mathcal{C}_{\mathrm{EF}}^{4}$, which carries the representation $\operatorname{Fun}\left(G_{f} / H\right)$, linear functions on the right cosets $G_{f} / H$. More precisely, $\operatorname{Fun}\left(G_{f} / H\right)$ consists of all linear functions on $G_{f}$, $f: G_{f} \rightarrow \mathbb{C}$, such that $f(h x)=f(x), \forall h \in H, x \in$ $G_{f}$ (takes the same value on a coset). The group action is the usual one on functions, $\rho_{\operatorname{Fun}\left(G_{f} / H\right)}(g)$ : $f(x) \mapsto f\left(g^{-1} x\right)$.

The linear maps $\alpha_{p, h}$ induced by the pure simple loops are all actions of group elements in $H$, and they are all identity maps on the special particle $\operatorname{Fun}\left(G_{f} / H\right)$. In other words, the bulk particle $\operatorname{Fun}\left(G_{f} / H\right)$ has trivial half-braiding with all the pure domain-wall strings. As a nonpure domain-wall string is just the bound state of $f$ with a pure domainwall string, its half-braiding with $\operatorname{Fun}\left(G_{f} / H\right)$ is also trivial. Thus, $\operatorname{Fun}\left(G_{f} / H\right)$ has trivial half-braiding with all the domain-wall strings. By the remote detectability condition (2), it must be the trivial particle carrying the trivial representation. In other words, we have $G_{f}=H$.

In conclusion, the pure simple loop excitations on the domain wall forms a group under fusion. It is exactly the same group $G_{f}$ whose representations are carried by the pointlike excitations in the bulk.

\section{Unitary fusion 2-category with a single invertible fermionic 1-morphism}

In addition to the strings on the domain wall discussed above, the domain wall also contains a single fermion with quantum dimension 1 . There are no other particles, as the domain wall is obtained via a maximal boson condensation. Summarizing the above results, we find that a $3+1 \mathrm{D} \mathrm{EF}$ topological order $\mathcal{C}_{\mathrm{EF}}^{4}$ has a domain wall between itself and 
the $2+1 \mathrm{D} Z_{2}^{f}$ topological order $\mathcal{C}_{Z_{1}^{f}}^{4}$. The domain wall is described by a unitary fusion 2-category $\mathcal{A}_{w}^{3}$. $\mathcal{A}_{w}^{3}$ has the following properties: Strings (objects) form a group under fusion, and the only nontrivial particle (1-morphism on the trivial string) is the fermion.

Besides the above necessary properties, $\mathcal{A}_{w}^{3}$ must also have the following property:

$$
Z\left(\mathcal{A}_{w}^{3}\right)=\mathcal{C}_{Z_{2}^{f}}^{4} \otimes\left(\mathcal{C}_{Z_{2}^{f}}^{4}\right)_{Z\left(\mathcal{A}_{w}^{3}\right)}^{\text {cen }} .
$$

Here, $Z\left(\mathcal{A}_{w}^{3}\right)$ is the bulk center of $\mathcal{A}_{w}^{3}$, and $\left(\mathcal{C}_{Z_{2}^{f}}^{4}\right)_{Z\left(\mathcal{A}_{w}^{3}\right)}^{\text {cen }}$ denotes the centralizer of $\mathcal{C}_{Z_{2}^{f}}^{4}$ in $Z\left(\mathcal{A}_{w}^{3}\right)$. The notion of the bulk center is introduced in Refs. [52,54], which is a generalization of the Drinfeld center to higher categories. Physically, $Z\left(\mathcal{A}_{w}^{3}\right)$ is the unique $3+1 \mathrm{D}$ topological order whose boundary can be $\mathcal{A}_{w}^{3}$. Since $\mathcal{A}_{w}^{3}$ is a domain wall between $\mathcal{C}_{Z_{2}^{f}}^{4}$ and $\mathcal{C}_{\mathrm{EF}}^{4}$, after folding, $\mathcal{A}_{w}^{3}$ can viewed as the boundary of the stacked topological order $\mathcal{C}_{Z_{2}^{f}}^{4} \otimes \mathcal{C}_{\mathrm{EF}}^{4}=$ $Z\left(\mathcal{A}_{w}^{3}\right)$ (strictly speaking, we should take the time reversal of the second component in the folding trick; but here $\mathcal{C}_{Z_{2}^{f}}^{4}$ is the same as its time reversal $\left.\overline{\mathcal{C}_{Z_{2}^{f}}^{4}}\right)$. Thus, $Z\left(\mathcal{A}_{w}^{3}\right)$ contains $\mathcal{C}_{Z_{2}^{f}}^{4}$ as a subcategory. The centralizer of $\mathcal{C}_{Z_{2}^{f}}^{4}$ in $Z\left(\mathcal{A}_{w}^{3}\right)$ is given by $\mathcal{C}_{\mathrm{EF}}^{4}=\left(\mathcal{C}_{Z_{2}^{f}}^{4}\right)_{Z\left(\mathcal{A}_{w}^{3}\right)}^{\text {cen }}$, and $Z\left(\mathcal{A}_{w}^{3}\right)$ must be the stacking of $\mathcal{C}_{Z_{2}^{f}}^{4}$ and its centralizer: $Z\left(\mathcal{A}_{w}^{3}\right)=\mathcal{C}_{Z_{2}^{f}}^{4} \otimes\left(\mathcal{C}_{Z_{2}^{f}}^{4}\right)_{Z\left(\mathcal{A}_{w}^{3}\right)}^{\text {cen }}$.

In conclusion, in this section, we give the properties of the domain wall obtained via maximal boson condensation in an EF topological order. Since the way to perform maximal boson condensation is unique, for each $\mathrm{EF}$ topological order, there is only one such domain wall, referred to as the unique canonical domain wall. Given such one-to-one correspondence between EF topological orders and their unique canonical domain walls, one can solve for all possible domain walls satisfying the above properties and thus classify the EF topological orders. However, it is better to work with boundaries, as we discuss in the next section.

\section{THE UNIQUE CANONICAL BOUNDARY OF 3 + 1D EF TOPOLOGICAL ORDERS}

Because the fusion 2-category on the domain wall of an $\mathrm{EF}$ topological order $\mathcal{C}_{\mathrm{EF}}^{4}$ and $Z_{2}^{f}$ topological order $\mathcal{C}_{Z_{2}^{f}}^{4}$ must satisfy the additional condition (24), it is hard to classify such a subset of fusion 2-categories. In this section, we construct the unique canonical boundary, also described by a unitary fusion 2-category, for every $3+1 \mathrm{D} \mathrm{EF}$ topological order. The bulk center of the unitary fusion 2-category of the canonical boundary directly gives the bulk $3+1 \mathrm{D}$ topological order. This way, we can focus on the fusion

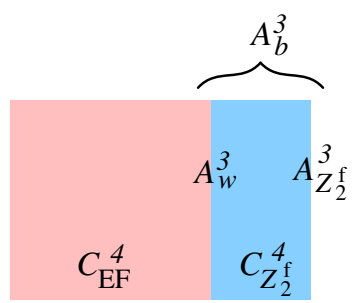

FIG. 12. $\mathcal{A}_{b}^{3}$ is the unique canonical boundary for $\mathcal{C}_{\mathrm{EF}}^{4} \cdot \mathcal{A}_{b}^{3}$ is formed by stacking the unique canonical domain wall $\mathcal{A}_{w}^{3}$ between $\mathcal{C}_{\mathrm{EF}}^{4}$ and $\mathcal{C}_{Z_{2}^{f}}^{4}$ and the boundary $\mathcal{A}_{Z_{2}^{f}}^{3}$ of $\mathcal{C}_{Z_{2}^{f}}^{4}$. Note that $\mathcal{A}_{w}^{3}$ and $\mathcal{A}_{Z_{2}^{f}}^{3}$ are separated by $\mathcal{C}_{Z_{2}^{f}}^{4}$.

2-category itself, without worrying about additional conditions like Eq. (24).

To construct the unique canonical boundary for a $3+1 \mathrm{D}$ $\mathrm{EF}$ topological order $\mathcal{C}_{\mathrm{EF}}^{4}$, we start with the unique canonical domain wall $\mathcal{A}_{w}^{3}$ between $\mathcal{C}_{\mathrm{EF}}^{4}$ and $\mathcal{C}_{Z_{2}^{f}}^{4}$ obtained by condensing all the bosonic pointlike excitations. We then create a boundary $\mathcal{A}_{Z_{2}^{f}}^{3}$ of $\mathcal{C}_{Z_{2}^{f}}^{4}$ by condensing the strings in $\mathcal{C}_{Z_{2}^{f}}^{4}$. Then, we make the intermediate $\mathcal{C}_{Z_{2}^{f}}^{4}$ very thin such that $\mathcal{A}_{w}^{3}, \mathcal{C}_{Z_{2}^{f}}^{4}$, and $\mathcal{A}_{Z_{2}^{f}}^{3}$ together can be viewed as a composite boundary.

That is to say, we consider the following boundary of $\mathcal{C}_{\mathrm{EF}}^{4}$ (see Fig. 12):

$$
\mathcal{A}_{b}^{3}=\mathcal{A}_{w}^{3} \otimes_{\mathcal{C}_{2}^{f}} \mathcal{A}_{Z_{2}}^{3}
$$

Equivalently, such a boundary $\mathcal{A}_{b}^{3}$ can be obtained by a onestep condensation, where all the bosons together with the $Z_{2}^{f}$ flux string $z$ are condensed.

Note that the domain wall $\mathcal{A}_{w}^{3}$ has stringlike excitations labeled by $G_{f}$. But the strings labeled by $Z_{2}^{f} \subset G_{f}$ can move across $\mathcal{C}_{Z_{2}^{f}}^{4}$ and then condense on the boundary $\mathcal{A}_{Z_{2}}^{3}$. So the stringlike excitations in the whole boundary $\mathcal{A}_{b}^{3}$ are labeled by $G_{f} / Z_{2}^{f} \equiv G_{b}$. All those strings have quantum dimension 1. Their fusion forms the group $G_{b}$. The boundary $\mathcal{A}_{b}^{3}$ also contains an invertible fermion $f$ with quantum dimension 1 . Such a pointlike excitation $f$ is inherited from $\mathcal{A}_{Z_{2}}^{3}, \mathcal{C}_{Z_{2}^{f}}^{4}$, and $\mathcal{A}_{w}^{3}$. The fermion $f$ can move freely between $\mathcal{A}_{Z_{2}}^{3}, \mathcal{C}_{Z_{2}^{f}}^{4}$, and $\mathcal{A}_{w}^{3}$.

We mention that a "pSC chain" (the 1D invertible fermionic topological order [63]) formed by the boundary fermions may attach to the strings discussed above. The pSC chain is invisible to the braiding between the stings and particles. But it doubles the types of strings. The end points of such pSC chains are the quantum-dimension- $\sqrt{2}$ Majorana zero modes. A more detailed discussion about this case is given later. 
Those considerations, after including the $\mathrm{pSC}$ chain and doubling the string types, allow us to obtain the following result: $\mathrm{A} 3+1 \mathrm{D} \mathrm{EF}$ topological order $\mathcal{C}_{\mathrm{EF}}^{4}$ has a unique canonical boundary $\mathcal{A}_{b}^{3}$, induced by a maximal boson condensation followed by a string condensation. $\mathcal{A}_{b}^{3}$ is described by a unitary fusion 2-category whose simple objects are labeled by $\hat{G}_{b}$, which is a $Z_{2}^{m}$ extension of $G_{b}$, where $Z_{2}^{m}$ labels the extra pSC chain string. The fusion of the objects is described by the group multiplication of $\hat{G}_{b}$. For each simple object (string), there is one nontrivial invertible 1-morphism corresponding to the fermion. There are also quantum-dimension- $\sqrt{2} 1$-morphisms (the Majorana zero modes) connecting two objects $g$ and $g m$, with $g \in \hat{G}_{b}$ and $m$ being the generator of $Z_{2}^{m}$.

On one hand, we show (together with more details given in the following sections) that $\mathcal{A}_{b}^{3}$ resulting from condensation must have the above properties. On the other hand, in Ref. [57], we give explicit constructions and show that all unitary fusion 2-categories with the above properties, or EF 2-categories for short, do give rise to $3+1 \mathrm{D}$ EF topological orders, which can be viewed as constructions of the bulk center $\mathcal{C}_{\mathrm{EF}}^{4}=Z\left(\mathcal{A}_{b}^{3}\right)$. Therefore, classifying EF 2-categories gives us a classification of $3+1 \mathrm{D}$ EF topological orders.

For clarity, we divide the EF topological orders into two types. If $\hat{G}_{b}=G_{b} \times Z_{2}^{m}$, the corresponding bulk topological orders are called EF1 topological orders. If $\hat{G}_{b}$ is a nontrivial extension of $G_{b}$ by $Z_{2}^{m}$, the corresponding bulk topological orders are called EF2 topological orders. Physically, the pSC chains in EF1 topological orders can be safely neglected, while in EF2 topological orders pSC chains have a nontrivial interplay with stringlike excitations and, thus, require more serious treatment.

We study EF1 topological order first. The canonical boundary $\mathcal{A}_{b}^{3}$ of EF1 topological order has a sub-2category $\overline{\mathcal{A}}_{b}^{3}$, where the pSC chains are dropped. We believe that $\overline{\mathcal{A}}_{b}^{3}$ encodes all the key information of $\mathcal{A}_{b}^{3}$. It is a pointed fusion 2-category as if the pSC chain is a trivial string: The information of the canonical boundary $\mathcal{A}_{b}^{3}$ of a $3+1 \mathrm{D}$ EF1 topological order $\mathcal{C}_{\mathrm{EF}}^{4}$ is encoded in a sub-2category $\overline{\mathcal{A}}_{b}^{3}$, which is a pointed unitary fusion 2-category whose simple objects are labeled by $G_{b}$. All simple objects and simple 1-morphisms are invertible. The fusion of the objects is described by the group multiplication of $G_{b}$. There is one nontrivial invertible fermionic 1-morphism for each object. Next, we give more details on the above statement.

\section{CLASSIFICATION OF EF1 TOPOLOGICAL ORDERS BY POINTED UNITARY FUSION 2-CATEGORIES}

\section{A. The canonical domain wall}

In this section, we consider the simpler case of EF1 topological orders. We start by describing the pointed unitary fusion 2-category $\overline{\mathcal{A}}_{w}^{3}$ on the domain wall. Such fusion 2-categories are special in the sense that their simple objects (corresponding to simple pure string types) and simple 1-morphisms are all invertible. The cases with noninvertible 1-morphisms are discussed later.

The content presented in this subsection is based on the results of Ref. [78], which gives a detailed definition of the tricategory (note that a fusion 2-category is a tricategory with a single object), simplified under the following assumptions.

(1) The identity (trivial string or trivial particle) -related data do not matter. The coherence relations involving both the associator or pentagonator and the identity-related data can be viewed as normalization conditions. We can set, by equivalent functors between fusion 2-categories or physically changing the basis or "gauge," all the identity-related data to be trivial; thus, the associator and the pentagonator are properly normalized.

(2) There are fermions on the strings, but fermions are not confined to the strings. Instead, fermions can move freely on the domain wall and even to the bulk. As a result, some of the particle-related data are fixed by fermionic statistics. We use $c(-,-)$ to denote the exchange statistics of the fermion:

$$
c(f, f)=-1, \quad c(\mathbf{1}, \mathbf{1})=c(\mathbf{1}, f)=c(f, \mathbf{1})=1 .
$$

In short, we assume that there is a convenient gauge choice such that some data of $\overline{\mathcal{A}}_{w}^{3}$ are either normalized or fixed by the fermionic statistics.

$\overline{\mathcal{A}}_{w}^{3}$ consists of the following data:

(1) Objects (pure string types). $-G_{f}$, a group that has a $Z_{2}$ central subgroup. The elements of $G_{f}$ label the simple pure strings.

(2) 1-morphisms (particles on strings).-For any simple pure string labeled by $g \in G_{f}$, we have $\operatorname{Hom}(g, g)=$ s Vec. In other words, we have particles live on a string $g$ which is viewed as a defect between the same type- $g$ string. $\operatorname{Hom}(g, g)=\mathrm{s} \mathcal{V e c}$ corresponds to the degenerate subspace or internal d.o.f. of the particle. Here, the particle is, in general, composite, which is formed by the accidental degeneracy of bosons and the fermion, which, in turn, gives rise to the super (i.e., $Z_{2}$ graded)-vectorspace sVec. We also have $\operatorname{Hom}(g, h)=0$ for $g \neq h \in G_{f}$, which means that there is no 1D defect between different simple pure strings. Simple 1-morphisms are denoted by $p_{g} \in \operatorname{Hom}(g, g)$, with a subscript to indicate its string type. $p$ values in $\{1, f\} \cong Z_{2}$ and follows a $Z_{2}$ fusion rule.

For convenience, we use the additive $\mathbb{Z}_{2}=\{0,1\}$ convention instead of the multiplicative $Z_{2}=\{\mathbf{1}, f\}$. 


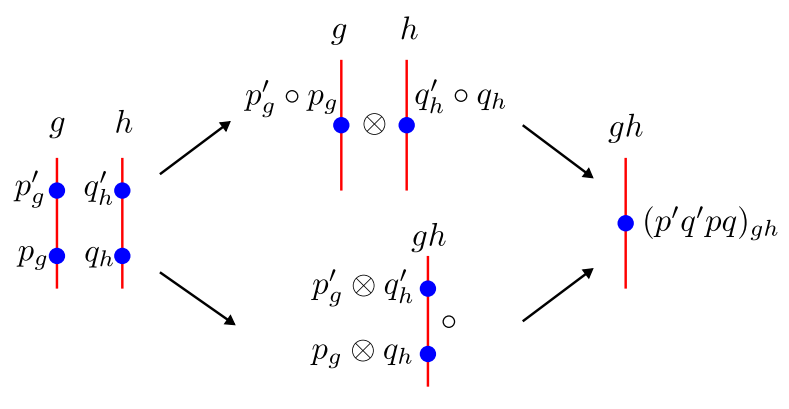

FIG. 13. The interchange law, corresponding to fusing four particles on two strings in different orders. The upper path and the lower path differ by a $U(1)$ phase $\tilde{b}\left(p_{g}{ }^{\prime}, q_{h}{ }^{\prime}, p_{g}, q_{h}\right)$.

$p=0$ corresponds to the trivial boson $\mathbf{1}$, and $p=1$ corresponds to the nontrivial fermion $f$. This way, the fermion exchange statistics is simply $c(p, q)=(-1)^{p q}$.

(3) 2-morphisms: Linear maps.-They correspond to deformations between various particle and string configurations.

(4) Fusion along strings, denoted by $p_{g} \circ p_{g}^{\prime}$ (composition of 1-morphisms but, in fact, is the tensor product in $\mathrm{s} \mathcal{V}$ ec ). - They follow the $\mathbb{Z}_{2}$ fusion rule for simple 1-morphisms, $p_{g} \circ p_{g}^{\prime}=\left(p+p^{\prime}\right)_{g}$.

(5) Fusion between strings, denoted by $\otimes$, for both objects (given by group multiplication) and 1-morphisms.-

$$
\begin{aligned}
g \otimes h & =g h, \quad g, h \in G_{f}, \\
p_{g} \otimes q_{h} & =(p+q)_{g h} .
\end{aligned}
$$

As we assume that particles (1-morphisms) can freely move on the domain wall, the fusion of 1-morphisms along different directions (along or between strings) should be essentially the same and independent of the string labels.

(6) The interchange law, a 2-isomorphism $\tilde{b}\left(p_{g}^{\prime}, q_{h}^{\prime}\right.$, $\left.p_{g}, q_{h}\right) \in U(1)$ (see Fig. 13).-

$$
\left(p_{g}^{\prime} \otimes q_{h}^{\prime}\right) \circ\left(p_{g} \otimes q_{h}\right) \cong\left(p_{g}^{\prime} \circ p_{g}\right) \otimes\left(q_{h}^{\prime} \circ q_{h}\right)
$$

on $\left(p^{\prime}+q^{\prime}+p+q\right)_{g h}$. In our case, the simple strings and simple particles are all invertible and have quantum dimension 1. Their degenerate subspaces are always one dimensional. Thus, the 2-isomorphisms are just $U(1)$ phase factors.

As particles can be freely detached from strings, we expect the above $U(1)$ phase independent of the string labels. Moreover, if we treat the fusion operations $\circ, \otimes$ as the same one, the difference between the two sides in Eq. (28) is just exchanging

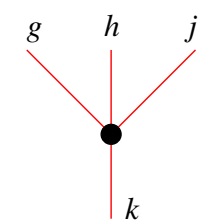

(a)

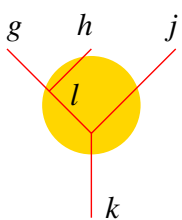

(b)

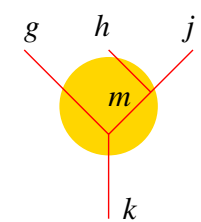

(c)
FIG. 14. (a) Fusion of strings $g, h, j$ gives rise to a defect between strings $g, h, j$ and string $k$. Two different ways of fusion, (b) and (c), may leads to different defects whose difference in particles is given by $n_{3}(g, h, j)$.

$q_{h}^{\prime}$ and $p_{g}$. Thus, to be consistent with fermionic statistics, we assume that

$$
\tilde{b}\left(p_{g}^{\prime}, q_{h}^{\prime}, p_{g}, q_{h}\right)=c\left(q^{\prime}, p\right)
$$

(7) Associator.-

(i) For $g, h, j \in G_{f}$, we have a 1-morphism $n_{3}(g, h, j):(g \otimes h) \otimes j \rightarrow g \otimes(h \otimes j)$, valuing in $\{\mathbf{1}, f\} \cong \mathbb{Z}_{2}$. See Fig. 14 .

(ii) We also have a 2-isomorphism $\tilde{n}_{3}\left(p_{g}, q_{h}, r_{j}\right) \in$ $U(1)$ to describe the $U(1)$ phase difference between two different orders to fuse strings and particles on the strings (see Fig. 15):

$$
\begin{aligned}
& n_{3}(g, h, j) \circ\left[\left(p_{g} \otimes q_{h}\right) \otimes r_{j}\right] \\
& \quad \cong\left[p_{g} \otimes\left(q_{h} \otimes r_{j}\right)\right] \circ n_{3}(g, h, j) .
\end{aligned}
$$

To be consistent with fermionic statistics, we assume that

$$
\tilde{n}_{3}\left(p_{g}, q_{h}, r_{j}\right)=c\left[n_{3}(g, h, j), p+q+r\right] .
$$

(8) Pentagonator.-For $g, h, j, k \in G_{f}, 2$-isomorphism $\nu_{4}(g, h, j, k) \in U(1):$

$$
\begin{aligned}
& {\left[\mathbf{1}_{g} \otimes n_{3}(h, j, k)\right] \circ n_{3}(g, h j, k) \circ\left[n_{3}(g, h, j) \otimes \mathbf{1}_{k}\right]} \\
& \quad \cong n_{3}(g, h, j k) \circ n_{3}(g h, j, k) .
\end{aligned}
$$

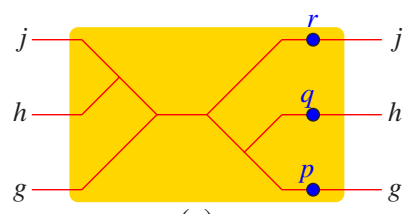

(a) (b)

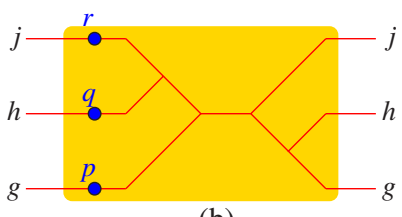

FIG. 15. The two domain-wall states in (a) and (b) may differ by a $U(1)$ phase $\tilde{n}_{3}\left(p_{g}, q_{h}, r_{j}\right)$ (see (31). 
These data satisfy the following.

(1) $n_{3}(g, h, j)$ is a normalized 3-cocycle in $H^{3}\left(G_{f}, \mathbb{Z}_{2}\right)$.

(2) For $g, h, j, k, l \in G_{f}$,

$$
\begin{aligned}
& \tilde{n}_{3}\left[n_{3}(g, h, j)_{g h j}, \mathbf{1}_{k}, \mathbf{1}_{l}\right] \tilde{n}_{3}\left[\mathbf{1}_{g}, n_{3}(h, j, k)_{h j k}, \mathbf{1}_{l}\right] \nu_{4}(h, j, k, l) \nu_{4}(g, h j, k, l) \nu_{4}(g, h, j, k l) \\
& =\tilde{n}_{3}\left[\mathbf{1}_{g}, \mathbf{1}_{h}, n_{3}(j, k, l)_{j k l}\right] \nu_{4}(g h, j, k, l) \nu_{4}(g, h, j k, l) \nu_{4}(g, h, j, k) .
\end{aligned}
$$

Note that

$$
\begin{aligned}
\tilde{n}_{3}\left[n_{3}(g, h, j)_{g h j}, \mathbf{1}_{k}, \mathbf{1}_{l}\right] & =c\left[n_{3}(g, h, j), n_{3}(g h j, k, l)\right] \\
& =(-1)^{n_{3}(g, h, j) n_{3}(g h j, k, l)},
\end{aligned}
$$

and similarly for other $\tilde{n}_{3}$ 's. We then have

$$
\frac{\nu_{4}(h, j, k, l) \nu_{4}(g, h j, k, l) \nu_{4}(g, h, j, k l)}{\nu_{4}(g h, j, k, l) \nu_{4}(g, h, j k, l) \nu_{4}(g, h, j, k)}=(-1)^{n_{3}(g, h, j) n_{3}(g h j, k, l)+n_{3}(g, h j k, l) n_{3}(h, j, k)+n_{3}(g, h, j k l) n_{3}(j, k, l)} .
$$

In other words, the 4-cochain $\nu_{4}(g, h, j, k)$ satisfies

$$
d \nu_{4}=(-)^{\mathrm{Sq}^{2} n_{3}}=(-)^{n_{3} \smile_{1} n_{3}}
$$

a relation first introduced in Ref. [67], where $\mathrm{Sq}^{2}$ is the Steenrod square and $\nu_{4}$ is normalized.

Here, "normalized" means that $n_{3}(g, h, j)=0$, if any of $g$, $h, k$ is $\mathbf{1}$, and $\nu_{4}(g, h, j, k)=1$, if any of $g, h, j, k$ is $\mathbf{1}$.

We point out that by now we consider the consistency conditions only on the domain wall. There are more constraints when we take into account the bulk; namely, the bulk center of the above fusion 2-category should be $\mathcal{C}_{\mathrm{EF}}^{4} \otimes \mathcal{C}_{Z_{2}^{f}}^{4}$ as in Eq. (24) - in particular, the fermion $f$ and the $Z_{2}^{f}$ flux $z$ must be liftable and form the $3+1 \mathrm{D} Z_{2}^{f}$ topological order $\mathcal{C}_{Z_{2}^{f}}^{4}$. Unfortunately, we do not have efficient algorithms or theorems to calculate bulk centers of fusion 2-categories, which makes it difficult to check under what extra conditions the bulk center of the above fusion 2-category has the desired form of Eq. (24). To avoid this weakness, we consider the canonical boundary instead as below.

\section{B. The canonical boundary}

We know that the $\mathcal{C}_{Z_{2}^{f}}^{4}$ topological order has a gapped boundary by condensing the $Z_{2}^{f}$ flux string $z$. On the gapped boundary, there is no string but only one nontrivial particle, the fermion. Imagine we have the gapped domain wall and gapped boundary as above, and between them is the intermediate $\mathcal{C}_{Z_{2}^{f}}^{4}$ phase. Now, we squeeze the intermediate $\mathcal{C}_{Z_{2}^{f}}^{4}$ phase to a very thin layer, such that we can view the composite domain-wall- $\overline{\mathcal{A}}_{w}^{3} / \mathcal{C}_{Z_{2}^{f}}^{4}$ /boundary- $\overline{\mathcal{A}}_{Z_{2}^{f}}^{3}$ together as a gapped boundary $\overline{\mathcal{A}}_{b}^{3}$ of $\mathcal{C}_{\mathrm{EF}}^{4}$. As a selfconsistency check, to see whether such a boundary does give rise to an EF topological order, we need only to check that in its bulk (the bulk center), the particles form $\operatorname{s} \mathcal{R e p}\left(G_{f}\right)$, which is much easier than checking the bulk center of the domain wall, which is valid only if it has the form of Eq. (24).

The composite boundary is described by a similar fusion 2-category as that for the domain wall. Most of the data and conditions discussed in the previous subsection apply. We list only the differences below.

(1) As the $z$ string condenses, the string types on the boundary are now labeled by $G_{b}=G_{f} / Z_{2}^{f}$. At the same time, the 2-cocycle $e_{2}(g, h) \in H^{2}\left(G_{b}, \mathbb{Z}_{2}\right)$ coming from the extension $Z_{2}^{f} \rightarrow G_{f} \rightarrow G_{b}$ arises in other data (see Fig. 16).
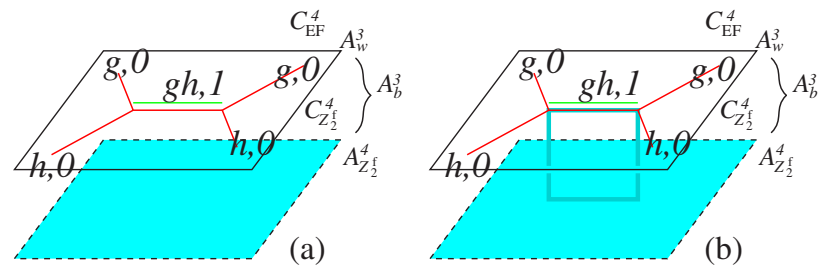

FIG. 16. (a) On the domain wall $\overline{\mathcal{A}}_{w}^{3}$, the strings are labeled by $(g, \mu) \in G_{f}$, where $g \in G_{b}$ and $\mu \in \mathbb{Z}_{2}^{f}$. The fusion of strings $(g, \mu)$ and $(h, \nu)$ is given by $(g, \mu) \otimes(h, \nu)=$ $\left(g h, \mu+\nu+e_{2}(g, h)\right)$. The group 2-cocycle $e_{2} \in H^{2}\left(G_{b}, \mathbb{Z}_{2}\right)$ gives rise to a $Z_{2}^{f}$ extension from $G_{b}$ to $G_{f}$. In the above graph, the string $(g, 0)$ is represented by a single line (red) and the string $(g, 1)$ a double line (red, green), where the extra (green) line can be viewed as the $Z_{2}^{f}$ flux line $z$. (b) Such a $Z_{2}^{f}$ flux line can be canceled by a $Z_{2}^{f}$ flux loop $z$ as indicated by the thick rectangular (blue) loop in the above graph. 
(2) When fusing $g, h$ on the composite boundary, $e_{2}(g, h)=1$ indicates that there is a $Z_{2}^{f}$ flux loop $z$ along the fused string $g h$ in the intermediate $\mathcal{C}_{Z_{2}^{f}}^{4}$ phase. As a result, the associator $\tilde{n}_{3}\left(p_{g}, q_{h}, r_{j}\right)$ needs to be modified. Under a certain framing convention (put the particles slightly below the string in Fig. 15 and slightly into the $\mathcal{C}_{Z_{2}^{f}}^{4}$ bulk), we find that (see Fig. 17)

$$
\tilde{n}_{3}\left(p_{g}, q_{h}, r_{j}\right)=(-1)^{n_{3}(g, h, j)(p+q+r)}(-1)^{r e_{2}(g, h)},
$$

where $(-1)^{n_{3}(g, h, j)(p+q+r)}$ is the fermion statistics (written in the additive $\mathbb{Z}_{2}$ convention) and $(-1)^{r e_{2}(g, h)}$ is the particle-loop statistics coming from $r$ going through the $Z_{2}^{f}$ flux loop $z$ along $g h$.

(3) $n_{3}(g, h, j)$ is now a 3 -cocycle in $H^{3}\left(G_{b}, \mathbb{Z}_{2}\right)$. The condition for $\nu_{4}$ is then modified to $\frac{\nu_{4}(h, j, k, l) \nu_{4}(g, h j, k, l) \nu_{4}(g, h, j, k l)}{\nu_{4}(g h, j, k, l) \nu_{4}(g, h, j k, l) \nu_{4}(g, h, j, k)}=(-1)^{e_{2}(g, h) n_{3}(j, k, l)}(-1)^{n_{3}(g, h, j) n_{3}(g h j, k, l)+n_{3}(g, h j k, l) n_{3}(h, j, k)+n_{3}(g, h, j k l) n_{3}(j, k, l)}$.

In other words, the 4-cochain $\nu_{4}(g, h, j, k) \in C^{4}\left[G_{b}, U(1)\right]$ satisfies

$$
d \nu_{4}=(-)^{n_{3} \smile n_{3}+e_{2} \smile n_{3}} .
$$

With these, one can check that in the bulk center bosonic particles form representations of $G_{b}$, and fermionic particles form projective representations of $G_{b}$ with a class described by $e_{2}$. Together, particles form nothing but $\operatorname{s} \mathcal{R e p}\left(G_{f}\right)$. So the above conditions for the composite boundary pass the self-consistency check; they do give rise to a $3+1 \mathrm{D}$ EF topological order. On one hand, the data $\left(G_{b}, e_{2}, n_{3}, \nu_{4}\right)$ together with their consistency conditions above are the necessary properties we show that the boundary $\overline{\mathcal{A}}_{b}^{3}$ of an EF1 topological order must have. On the other hand, these properties are also sufficient for the bulk center of $\overline{\mathcal{A}}_{b}^{3}$ to be an EF1 topological order. Thus, we have a classification of $3+1 \mathrm{D}$ EF1 topological orders, by $\overline{\mathcal{A}}_{b}^{3}$ with these properties, namely, by $\left(G_{b}, e_{2}, n_{3}, \nu_{4}\right)$, where $e_{2} \in H^{2}\left(G_{b}, \mathbb{Z}_{2}\right), n_{3} \in H^{3}\left(G_{b}, \mathbb{Z}_{2}\right), \nu_{4} \in C^{4}\left[G_{b}, U(1)\right]$ satisfying Eq. (39). The above has the same form as the group supercohomology theory for fermionic SPTs. Recently, a
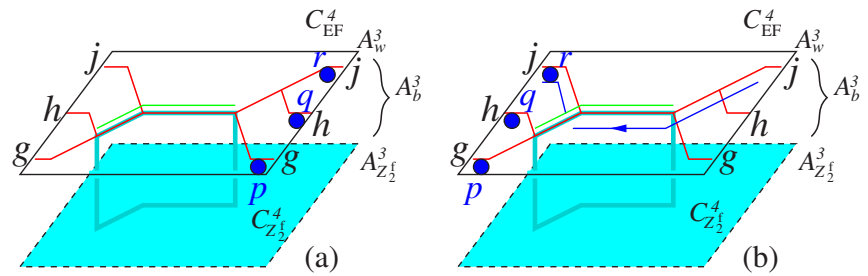

FIG. 17. The two domain-wall states in (a) and (b) may differ by a $U(1)$ phase $\tilde{n}_{3}\left(p_{g}, q_{h}, r_{j}\right)$ [see Eq. (37)]. The string label $(g, 0)$ on $\overline{\mathcal{A}}_{w}$ is abbreviated to $g$. This figure shows the case that $e_{2}(g, h)=e_{2}(g, h j)=1, e_{2}(g h, j)=e_{2}(h, j)=0$. new class of $3+1 \mathrm{D}$ fermionic SPTs was found, by "decorating Kitaev's Majorana chains" [79,80], which is beyond group supercohomology. In the next section, we show that such a "Kitaev's Majorana chain decoration" also enters the classification of topological orders.

For completeness, let us briefly discuss the equivalence relation for the above data. First, $G_{b}$ together with $e_{2}$ is the same data as the group $G_{f}$. Since the particles form $\operatorname{s} \mathcal{R e p}\left(G_{f}\right)$, by the Tannaka duality $\left(G_{b}, e_{2}\right)$ is fully determined up to group isomorphisms. However, $\left(n_{3}, \nu_{4}\right)$ admits more gauge transformations than coboundaries. The key is to note that $d \nu_{4}$ depends on $n_{3}$; thus, if $n_{3}$ is changed by a coboundary $d u_{2}$, to keep Eq. (39), $\nu_{4}$ must be modified by an extra cochain $w_{4}$ which satisfies

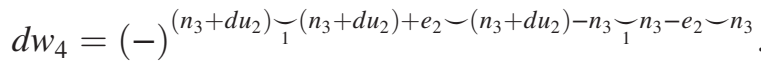

Such $w_{4}$ needs to be determined only up to coboundaries. To fix the cocycle ambiguity, note that $w_{4}$ must be 0 when $u_{2}=0$. Then, by a straightforward calculation we find that

$$
w_{4}=(-1)^{n_{3} \smile d u_{2}+u_{2} \smile u_{2}+u_{2} \smile d u_{2}+e_{2} \smile u_{2}} .
$$

Therefore, in general, for any 2-cochain $u_{2} \in C^{2}\left(G_{b}, \mathbb{Z}_{2}\right)$ and 3-cochain $\eta_{3} \in C^{3}\left[G_{b}, U(1)\right]$,

$$
\begin{aligned}
& n_{3} \rightarrow n_{3}+d u_{2}, \\
& \nu_{4} \rightarrow \nu_{4} \times d \eta_{3} \times(-1)^{n_{3} \smile d u_{2}+u_{2} \smile u_{2}+u_{2} \smile d u_{2}+e_{2} \smile u_{2}}
\end{aligned}
$$

is a gauge transformation and gives an equivalent solution. 
Note that $(-1)^{n_{3} \smile 2} d u_{2}+u_{2} \smile u_{2}+u_{2} \smile d u_{2}+e_{2} \smile u_{2}$ is, in general, a 4-cochain, and $d \nu_{4}$ is shifted under such a gauge transformation. If we fix $n_{3}$, namely, let $d u_{2}=0$, $u_{2} \in Z^{2}\left(G_{b}, \mathbb{Z}_{2}\right), \nu_{4}$ transforms as

$$
\nu_{4} \rightarrow \nu_{4} \times d \eta_{3} \times(-1)^{u_{2}-u_{2}+e_{2} \smile u_{2}},
$$

where $(-1)^{u_{2} \smile u_{2}+e_{2} \smile u_{2}}$ is now a 4-cocycle but may not be the trivial one. We see that $\nu_{4}$ is, in fact, classified by (forms a torsor over) the group $H^{4}\left[G_{b}, U(1)\right] / \Gamma$, where $\Gamma$ is the subgroup generated by $(-1)^{u_{2} \smile u_{2}+e_{2}-u_{2}}$ for all 2-cocycles $u_{2}$.

Besides the gauge transformations, different $n_{3}, \nu_{4}$ are also equivalent if they can be related by (outer) group isomorphisms of $G_{f}$ or $\left(G_{b}, e_{2}\right)$ (which can be followed by gauge transformations). We note that this part is different from the equivalence relation of fermionic SPT phase, where the symmetry group $G_{f}$ should always be fixed.

To "add up" two solutions $\left(n_{3}, \nu_{4}\right)$ and $\left(n_{3}^{\prime}, \nu_{4}^{\prime}\right)$, one also needs to follow a twisted rule:

$$
\left(n_{3}, \nu_{4}\right)+\left(n_{3}^{\prime}, \nu_{4}^{\prime}\right)=\left(n_{3}+n_{3}^{\prime}, \nu_{4} \nu_{4}^{\prime}(-1)^{n_{3}{ }_{2}^{\prime}}\right) .
$$

The above is the best we can do for equivalence relations of EF1 topological orders. But, we point out that they are only sufficient conditions for different sets of data to be equivalent. Two sets of data which are seemingly nonequivalent according to the above may be related by some extra gauge transformations and are, in fact, equivalent. To see what is missing, we may consider EF1 topological orders as special EF topological orders with some extra data, such as $m_{2}$, being trivial. These extra data, although trivial, still allow extra gauge transformations on them and may generate nontrivial equivalence relations.

In more rigorous categorical language, the equivalence relation of a bulk topological order is given by the Morita equivalence of the unitary fusion 2-category describing the gapped boundary, namely, $Z\left(\mathcal{A}_{b}^{3}\right) \simeq Z\left(\mathcal{B}_{b}^{3}\right)$. For the canonical boundaries $\mathcal{A}_{b}^{3}, \mathcal{B}_{b}^{3}$, obtained by condensing particular excitations, $Z\left(\mathcal{A}_{b}^{3}\right) \simeq Z\left(\mathcal{B}_{b}^{3}\right)$ implies $\mathcal{A}_{b}^{3} \simeq \mathcal{B}_{b}^{3}$. Group automorphisms plus the gauge transformations listed above generate only a subset of all possible equivalence functors between $\mathcal{A}_{b}^{3}$ and $\mathcal{B}_{b}^{3}$. We leave the complete equivalence relation for future work.

\section{CLASSIFICATION OF EF TOPOLOGICAL ORDERS BY EF 2-CATEGORIES ON THE CANONICAL BOUNDARY}

In this section, we shift to more general EF topological order. Mathematically, we drop the assumption that all simple excitations are invertible and study what else is possible. We then find that the only remaining possibility is an extra $Z_{2}$ string together with its end points with quantum dimension $\sqrt{2}$. Physically, we identify such an extra string as the pSC chain and its end point as a Majorana zero mode.
We then seriously consider the nontrivial interplay between pSC chains and stringlike excitations.

\section{A. Define string type using local or nonlocal unitary transformations?}

In the above discussions, we omit the possibility that between different strings there can be defects or 1-morphisms. This omission is a consequence of defining the type of stringlike excitations up to nonlocal perturbations along the string (see Sec. III B). To see this point, let us consider that a loop consists of two string segments labeled by $g, h$ connected by two pointlike defects (i.e., 1-morphisms) $\sigma \in \operatorname{Hom}(g, h), \sigma^{\prime} \in \operatorname{Hom}(h, g)$ (see Fig. 18). Under nonlocal perturbations, the loop can become a $g$ loop carrying $\sigma \circ \sigma^{\prime} \in \operatorname{Hom}(g, g)$ or an $h$ loop carrying $\sigma^{\prime} \circ \sigma \in \operatorname{Hom}(h, h)$. Thus, $g$ and $h$ are equivalent under nonlocal perturbations along the string.

In the fusion 2-category, the objects or strings and 1-morphisms or pointlike defects are actually defined up to local unitary transformations. Moreover, if there exists an invertible 1-morphism (namely, a pointlike defect with quantum dimension 1) between two objects (namely, two string segments), such two objects are equivalent in the fusion 2-category. Therefore, if some $\sigma \in \operatorname{Hom}(g, h)$ is an invertible 1-morphism, then $g$ and $h$ are indeed equivalent as objects in the fusion 2-category, which is consistent with the nonlocal perturbation point of view. However, it is possible that no 1-morphism in $\operatorname{Hom}(g, h)$ is invertible, and $g, h$ are not equivalent in the fusion 2-category. To include this possibility, we introduce a different equivalent relation of strings, using local unitary transformations plus invertible 1-morphisms, which is consistent with that in the fusion 2-category: Two strings defined under local unitary transformations are called of the same local type if there is an invertible 1-morphism between them. (Section III B defines types of strings as equivalence classes of nonlocal unitary transformations, which is different from local types of string defined as equivalence classes of local unitary transformations.) The set of local types is denoted by $\hat{G}_{b}$. We have already shown that the string types defined via nonlocal unitary transformations form a group $G_{b}$. Clearly, $\left|\hat{G}_{b}\right| \geq\left|G_{b}\right|$, and two different local types may correspond to the same type.

With the expanded string local types defined by local unitary transformation, our arguments in Sec. V are still valid, which shows that, on the boundary, closed strings

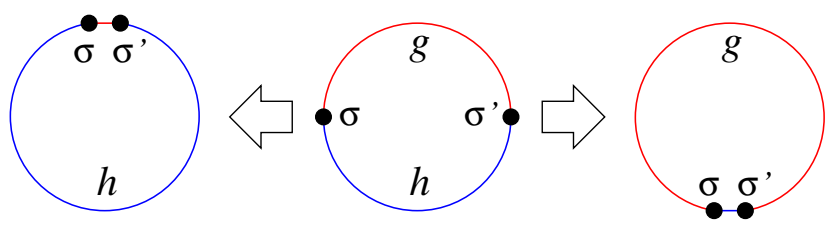

FIG. 18. If two strings $g$ and $h$ can be connected by a domain wall (i.e., an 1-morphism), then, under nonlocal unitary transformations, strings $g$ and $h$ will be equivalent. 
have quantum dimension 1 and form a group under fusion. $\hat{G}_{b}$ is actually a group that describes the fusion of the local types. Also, using the half-braiding with the pointlike excitation in the bulk (see Sec. V), we can assign each boundary string local type a group element in $G_{b}$. Thus, there is a group homomorphism $\hat{G}_{b} \rightarrow{ }^{m} \rightarrow G_{b}$. If there are noninvertible 1-morphisms between different local types, they can together form a closed loop and must be assigned the same element in $G_{b}$. In fact, the string types up to nonlocal perturbations are just local types further up to noninvertible 1-morphisms. Indeed, $G_{b}$ is a quotient group of $\hat{G}_{b}$ by imposing equivalent relations via noninvertible 1-morphisms.

\section{B. New string type from pSC chain}

Next, we carefully examine what possible noninvertible 1-morphisms can there be and their physical meaning. Since all the local types of strings labeled by $g \in \hat{G}_{b}$ have quantum dimension 1 and form a group under fusion, the 1-morphisms automatically obtain a grading by this group; namely, $p \in \operatorname{Hom}(g, h)$ is graded by $h g^{-1}$. As a result of such grading, the total quantum dimension of nonempty $\operatorname{Hom}(g, h)$ must be the same. In our previous work discussing $\mathrm{AB}$ topological orders [37], $\operatorname{dim} \operatorname{Hom}(g, h)=$ $\operatorname{dim} \operatorname{Hom}(g, g)=1$, and, thus, $\operatorname{Hom}(g, h)$ can allow only one simple invertible 1-morphism or be empty; in this case, nonempty $\operatorname{Hom}(g, h)$ just implies $g=h$. In other words, in $\mathrm{AB}$ topological orders, there is no room for simple noninvertible 1-morphisms on the canonical boundary. It also means that, on the canonical boundary of the AB topological, the string local types defined using local unitary transformations plus invertible 1-morphisms and the string types defined using nonlocal unitary transformations are the same; i.e., $\hat{G}_{b}=G_{b}$.

However, for EF topological orders, it is not the case. Since $\operatorname{Hom}(g, g)=\mathrm{s} \mathcal{V}$ ec, if $\operatorname{Hom}(g, h)$ is not empty for certain $g, h$, we have $\operatorname{dim} \operatorname{Hom}(g, h)=\operatorname{dim} \operatorname{Hom}(g, g)=$ $\operatorname{dim}(\mathrm{s} \mathcal{V}$ ec $)=2$, which means that there can be one simple noninvertible 1-morphism with quantum dimension $\sqrt{2}$. In this case, $\left|\hat{G}_{b}\right|>\left|G_{b}\right|$.

We can fuse a $g^{-1}$ string to this simple noninvertible 1-morphism between $g, h$ and obtain a simple noninvertible 1 -morphism in $\operatorname{Hom}\left(g g^{-1}, h g^{-1}\right)=\operatorname{Hom}\left(\mathbf{1}, h g^{-1}\right)$. Let such $h g^{-1} \equiv m$ and denote the noninvertible 1-morphism by $\sigma_{m} \in \operatorname{Hom}(\mathbf{1}, m)$. It is easy to see that, for any string $k$, $\sigma_{m} \otimes \mathbf{1}_{k}$ is a noninvertible 1-morphism in $\operatorname{Hom}(k, m k)$. In fact, such an $m$ string generates the kernel of the projection $\pi^{m}: \hat{G}_{b} \rightarrow G_{b}$.

We find the following properties of such strings.

(1) $m$ is a $Z_{2}$ string, $m^{2}=1$.- Consider fusing two $\sigma_{m}$. We obtain $\sigma_{m} \otimes \sigma_{m} \in \operatorname{Hom}\left(\mathbf{1}, m^{2}\right)$, whose quantum dimension is 2 . It can only split as the direct sum of two invertible 1-morphisms, which implies that the $m^{2}$ string and $\mathbf{1}$ are equivalent.
(2) $m$ is unique.-Suppose that there is another simple noninvertible $\sigma_{m^{\prime}} \in \operatorname{Hom}\left(\mathbf{1}, m^{\prime}\right)$. Using the same trick, we see that $\sigma_{m} \otimes \sigma_{m^{\prime}} \in \operatorname{Hom}\left(\mathbf{1}, \mathrm{mm}^{\prime}\right)$ is the direct sum of two invertible 1-morphisms. Thus, $m m^{\prime}=\mathbf{1}$. Together with $m^{2}=\mathbf{1}$, we conclude that $m=m^{\prime}$.

(3) $m$ is central, $\forall g, m g=g m$.-To see this property, consider $\mathbf{1}_{g} \otimes \sigma_{m} \otimes \mathbf{1}_{g^{-1}}$, which is a simple noninvertible 1-morphism in $\operatorname{Hom}\left(g g^{-1}, g m g^{-1}\right)=$ $\operatorname{Hom}\left(\mathbf{1}, g m g^{-1}\right)$. Since $m$ is unique, we must have $m=g m g^{-1}$.

Therefore, it is possible to have a $Z_{2}$ string $m$ which can be open on the canonical boundary of EF topological orders. Its end points [simple noninvertible 1-morphisms in $\operatorname{Hom}(\mathbf{1}, m)$ ] have quantum dimension $\sqrt{2}$.

Physically, the $m$ string is distinguished from the trivial string under the equivalences generated by local unitary transformations. In other words, the $m$ string and trivial string have different local types. But they have the same type; namely, the $m$ string becomes the same as the trivial string under the equivalences generated by nonlocal unitary transformations. This result implies that $m$ is a descendant string formed by lower-dimensional topological excitations (since it can have a boundary). On the boundary of an EF topological order, the only lower-dimensional topological excitations are the trivial particles and the fermions. Since there is no topological order in $1 \mathrm{D}$, the trivial particles cannot form any nontrivial strings. On the other hand, the fermions can form a topological pSC chain. Thus, the $m$ string must be a pSC chain. The simple 1-morphism between the $m$ string and trivial string in $\operatorname{Hom}(\mathbf{1}, m)$ (i.e., the end point of the $m$ string) is the Majorana zero mode at the end of the pSC chain.

We emphasize here that such an extra string $m$ and noninvertible 1-morphism $\sigma_{m}$ are the only remaining possibilities beyond the pointed case discussed in Sec. VII. The boundary strings are labeled by a larger group $\hat{G}_{b}$, which is a central $Z_{2}$ extension of $G_{b}$ :

$$
\{\mathbf{1}, m\} \equiv Z_{2}^{m} \rightarrow \hat{G}_{b} \stackrel{\pi^{m}}{\rightarrow} G_{b} .
$$

The unitary fusion 2-category $\mathcal{A}_{b}^{3}$ on such a boundary is thus an EF 2-category as defined in Sec. II C.

We note that the elements in $\hat{G}_{b}$ can be labeled by $\left(g^{b}, x\right)$, $g^{b} \in G_{b}$ and $x \in Z_{2}=\{1,-1\}$. The multiplication in $\hat{G}_{b}$ is given by

$$
\left(g^{b}, x\right)\left(h^{b}, y\right)=\left(g^{b} h^{b}, m_{2}\left(g^{b}, h^{b}\right) x y\right),
$$

where $m_{2}\left(g^{b}, h^{b}\right)$ is a group 2-cocycle in $H^{2}\left(G_{b}, Z_{2}\right)$. Without losing generality, we choose $(1,1)$ to label the trivial string and $(1,-1)$ to label the $m$ string, which is equivalent to choosing a normalized 2-cocycle, $m_{2}\left(1, g^{b}\right)=$ $m_{2}\left(g^{b}, 1\right)=1, \forall g^{b} \in G_{b}$. With the enlarged boundary 
string local types and noninvertible 1-morphisms, EF topological orders are classified by EF 2-categories $\mathcal{A}_{b}^{3}$.

\section{C. $Z_{2}^{f}$ topological order cannot be an EF2 topological order}

After rigorously introducing the local type of strings and pSC chains, we now fix a weak point in the arguments in Sec. III E. In Sec. III E, we described a 3+1D EF1 topological order $\mathcal{C}_{Z_{2}^{f}}^{4}$, whose pointlike excitations are described by $\operatorname{sRep}\left(Z_{2}^{f}\right)$ [38]. Such a topological order has nontrivial particle type $f$, which is a fermion, and two nontrivial string types $s_{1}$ and $s_{2}$. The fusion rule between the loops $s_{1}$ and $s_{2}$ and the fermion $f$ is given by

$$
\begin{aligned}
f \otimes f & =\mathbf{1}, \quad f \otimes s_{1}=s_{2}, \quad f \otimes s_{2}=s_{1}, \\
s_{1} \otimes s_{1} & =s_{2} \otimes s_{2}=\mathbf{1}, \quad s_{1} \otimes s_{2}=f .
\end{aligned}
$$

In $s_{1} \otimes s_{1}=\mathbf{1}, \mathbf{1}$ means the trivial type of string defined by a nonlocal unitary transformation. However, here we want this 1 to be also the trivial local type of string defined by a local unitary transformation. In the following, we show this by contradiction.

Let us assume that $s_{1} \otimes s_{1}=\mathbf{1}$ is a nontrivial local type of string that corresponds to a loop of the pSC chain with an even number of fermions. We denote such a loop by $m_{e}$. Thus, we have $s_{1} \otimes s_{1}=m_{e}$. This result implies that there are four pure string loops labeled by $\mathbf{1}, s_{1}, m_{e}$, and $\bar{s}_{1}$. Their fusion is described by the $Z_{4}$ group:

$s_{1} \otimes s_{1}=m_{e}, \quad s_{1} \otimes s_{1} \otimes s_{1}=\bar{s}_{1}, \quad s_{1} \otimes \bar{s}_{1}=\mathbf{1}$.

Thus, the pure strings are described by $\hat{G}_{f}=$ $Z_{2}^{m} \lambda_{m_{2}^{f}} Z_{2}^{f}=Z_{4}$. Since $m_{2}^{f}$ is nontrivial, the corresponding topological order is an EF2 topological order, denoted by $\hat{\mathcal{C}}_{Z_{2}^{f}}^{4}$ hereafter.

Fusing with the fermion $f$ gives us another set of string loops $f, s_{2}, m_{o}$, and $\bar{s}_{2}$ :

$$
f \otimes s_{1}=s_{2}, \quad f \otimes m_{e}=m_{o}, \quad f \otimes \bar{s}_{1}=\bar{s}_{2} .
$$

Now let us consider the dimension reduction of the $3+$ $1 \mathrm{D}$ topological order $\hat{\mathcal{C}}_{Z_{2}^{f}}^{4}$ to a $2+1 \mathrm{D}$ topological order. In the untwisted sector, we obtain a $2+1 \mathrm{D}$ topological order $\mathcal{C}_{\text {untw }}^{3}$ which is the $Z_{2}$ gauge theory (or the Drinfeld center $\left.Z\left[\operatorname{s} \mathcal{R e p}\left(Z_{2}^{f}\right)\right]\right)$ described by particles $\mathbf{1}, e, m, \psi=e m$. In the dimension reduction, the excitations in $\hat{\mathcal{C}}_{Z_{2}^{f}}^{4}$ reduce to the particles in $Z\left[\operatorname{s} \mathcal{R e p}\left(Z_{2}^{f}\right)\right]$ (see Fig. 3):

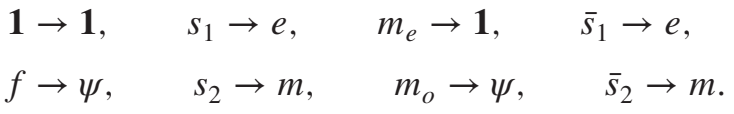

One can check that the fusion of $\mathbf{1}, s_{1}, m_{e}, \bar{s}_{1}, f, s_{2}, m_{o}, \bar{s}_{2}$ reproduces the fusion of $\mathbf{1}, e, m, \psi$, which is described by group $Z_{2} \times Z_{2}$.

In the twisted sector characterized by the base string $s_{1}$, assume the dimension reduction of $s_{1}$ is $a: s_{1} \rightarrow a$ (see Fig. 7), where $a$ is a particle in the dimension-reduced $2+1 \mathrm{D}$ topological order $\mathcal{C}_{s_{1}}^{3}$. A pSC chain loop $m_{e}$, when wrapping around a $s_{1}$ string (the $Z_{2}^{f}$ flux), carries an odd number of fermions. Thus, the dimension reduction of $m_{e}$ gives us a fermion $\psi: m_{e} \rightarrow \psi$. Therefore, we have

$a \otimes a=\psi, \quad a \otimes \bar{a}=\mathbf{1}, \quad \psi \otimes \psi=\mathbf{1}, \quad a \otimes a \otimes a=\bar{a}$.

We see that the fusion of $1, a, \psi, \bar{a}$ is described by a $Z_{4}$ group. The fermion $f$ in $\hat{\mathcal{C}}_{Z_{2}^{f}}^{4}$ reduces to the fermion $\psi$ in $\mathcal{C}_{s_{1}}^{3}$. This reduction allows us to obtain the dimension reduction

$\mathbf{1} \rightarrow \mathbf{1}, \quad s_{1} \rightarrow a, \quad m_{e} \rightarrow \psi, \quad \bar{s}_{1} \rightarrow \bar{a}$,

$f \rightarrow \psi, \quad s_{2} \rightarrow \bar{a}, \quad m_{o} \rightarrow \mathbf{1}, \quad \bar{s}_{2} \rightarrow a$.

We see that the dimension-reduced $2+1 \mathrm{D}$ topological order $\mathcal{C}_{s_{1}}^{3}$ has four particle types with $Z_{4}$ fusion. There are only four possibilities for such Abelian $2+1 \mathrm{D}$ topological order, described $K$ matrices $[81,82] K= \pm 4$ and

$$
K= \pm\left(\begin{array}{lll}
2 & 1 & 1 \\
1 & 2 & 1 \\
1 & 1 & 2
\end{array}\right)
$$

They have chiral central charges $c= \pm 1, \pm 3 \bmod 8$ and are not Drinfeld centers, which contradicts with the result obtained in Sec. III D that the dimension-reduced topological order is always a Drinfeld center. Therefore, the assumed $3+1 \mathrm{D}$ EF2 topological order $\hat{\mathcal{C}}_{Z_{2}^{f}}^{4}$ does not exist. This result supports our conclusion that condensing all the bosonic pointlike excitations produces a unique $Z_{2}^{f}$ topological order obtained in Sec. III.

\section{Partial characterization of EF 2-categories}

Next, we discuss in more detail how the extra string $m$ and noninvertible 1-morphism $\sigma_{m}$ affect the characterization of the fusion 2-categories.

Now, strings are labeled by a larger group $\hat{G}_{b}$ on the canonical boundary. But note the fact that the data and conditions not involving $\sigma_{m}$ are not affected at all. That is to say, the enlarged EF 2-category always contain a pointed sub-2-category with data $\left(\hat{G}_{b}, \hat{e}_{2}, \hat{n}_{3}, \hat{\nu}_{4}\right)$ which describes an EF1 topological order as in Sec. VII.

In other words, there is a map from EF 2-categories $\mathcal{A}_{b}^{3}$ that classify EF topological orders to the pointed unitary fusion 2-categories $\tilde{\mathcal{A}}_{b}^{3}$ that classify EF1 topological orders. 
Such a map sends an EF 2-category $\mathcal{A}_{b}^{3}$ with simple objects $\hat{G}_{b}$ to a pointed unitary fusion 2-category $\tilde{\mathcal{A}}_{b}^{3}$ also with simple objects $\hat{G}_{b}$, by taking the pointed sub-2-category (ignoring the noninvertible 1-morphisms). Therefore, there is a map from $\mathrm{EF}$ topological orders to $\mathrm{EF} 1$ topological orders, which sends an EF topological order with pointlike excitations described by $\operatorname{s} \operatorname{Rep}\left(Z_{2}^{f} \lambda G_{b}\right)$ to an $\mathrm{EF} 1$ topological order with pointlike excitations described by $\operatorname{s} \mathcal{R e p}\left(Z_{2}^{f} \lambda \hat{G}_{b}\right)$.

Such a map is clearly not invertible. One cannot expect that, for every pointed unitary fusion 2-category, noninvertible 1-morphisms $\sigma_{m}$ can be added consistently to obtain an EF 2-category. There must be some additional constraints for this to be done.

First, the $\sigma_{m}$ 1-morphism must itself satisfy some additional braiding and fusion constraints, which means that $\hat{\tilde{b}}(\bullet, \bullet, \bullet, \bullet)$ and $\hat{\tilde{n}}_{3}(\bullet, \bullet, \bullet)$ involving $\sigma_{m}$ take different forms. We expect that the results are closely related to the braiding statistics of Ising anyons.

Second, the strings of local types $g$ and $g m$ can be "connected" by noninvertible 1-morphisms; moreover, when $m_{2}$ is nontrivial, $g$ and $g m$ are, in principle, not distinguishable, which implies, for example, that $\hat{n}_{3}(g, h, j)$ and $\hat{n}_{3}(g m, h, j)$, or $\hat{\nu}_{4}(g, h, j, k)$ and $\hat{\nu}_{4}(g, h m, j m, k)$, etc., are related by $m$ and $\sigma_{m}$. Thus, we conjecture that $\hat{n}_{3}, \hat{\nu}_{4}$ can be factorized. If we split the $\hat{G}_{b}$ variables $g_{i}$ into $G_{b}$ parts and $Z_{2}^{m}$ parts as in Eq. (45), $g_{i}=\left(g_{i}^{b}, x_{i}\right)$, and view $x$ as a 1 -cochain with values $x_{i}$, we can formally express the multiplication as

$$
d x=m_{2}\left(g_{i}\right)
$$

We conjecture that $\hat{n}_{3}$ has the form

$$
\hat{n}_{3}\left(g_{i}^{b}, x_{i}\right)=n_{3}\left(g_{i}^{b}\right)+m_{2} \smile x+e_{2} \smile x,
$$

where $n_{3}$ is a cochain in $C^{3}\left(G_{b}, \mathbb{Z}_{2}\right)$. Then, the fact that $\hat{n}_{3}$ is a cocycle in $\hat{G}_{b}, d \hat{n}_{3}=0$, implies

$$
d n_{3}=m_{2} \smile m_{2}+e_{2} \smile m_{2}=\mathrm{Sq}^{2} m_{2}+e_{2} \smile m_{2},
$$

which agrees with the formulas in Refs. [79,80] when $e_{2}=0$.

Similarly, we expect that $\hat{\nu}_{4}\left(g_{i}^{b}, x_{i}\right)=\nu_{4}\left(g_{i}^{b}\right) \nu_{m}\left(g_{i}^{b}, x_{i}\right)$, where $\nu_{4}$ is a 4-cochain in $G_{b}$ and $\nu_{m}$ is a factor depending both on $g_{i}^{b}$ and on how the $m$ string is attached (namely, $x_{i}$ ). Then, from

$$
d \hat{\nu}_{4}=(-)^{\hat{n}_{3} \smile \hat{n}_{3}+\hat{e}_{2}-\hat{n}_{3}}
$$

one can derive the condition that $\nu_{4}$ satisfies. However, there seems no simple formula for $\nu_{4}$ (see those in Refs. [79,80,83,84]), and we have no good conjecture on the form of $\nu_{m}$ and the resulting condition for $\nu_{4}$.
Nevertheless, the first few terms obtained by expanding $\hat{n}_{3}$ in Eq. (55) already agree with those in Ref. [79]. We believe that by thoroughly studying the structures of EF 2-categories a complete formula can eventually be obtained. On the other hand, the formulas derived using other independent approaches [79,80,83,84] may help understanding EF 2-categories.

We leave the details of the additional constraints involving the noninvertible 1-morphism $\sigma_{m}$ for future work (see e.g., Ref. [57]). We believe that they also have the same form as those for fermionic SPTs with the decoration of Kitaev's Majorana chain $[79,80]$.

\section{E. Majorana zero modes at triple-string junctions}

In the following, we describe a bulk property that allows us to distinguish between EF1 and EF2 topological orders. In particular, we design a setup which allows us to use the appearance of the Majorana zero mode to directly measure the cohomology class of $m_{2}$. For simplicity, let us assume $G_{f}$ to be Abelian for the time being. In this case, the different types of bulk strings are labeled by $g^{f} \in G_{f}$. In our setup, we first choose a fixed set of trapping potentials that trap a fixed set of strings labeled by $g^{f} \in G_{f}$. Note that the different strings in the set can all be distinguished by their different braiding properties with the pointlike excitations. Then, choosing three strings from such a fixed set, we can form the configuration in Fig. 19(a). For Abelian $G_{f}$, one may expect that the degeneracy for the configuration in Fig. 19(a) to be 1. In the following, we show that sometimes the configuration in Fig. 19(a) has a twofold topological degeneracy. By measuring which triples $g_{1}^{f}, g_{2}^{f}, g_{3}^{f}$ in the fixed set of strings give rise to twofold topological degeneracy, we can determine the cohomology class of $m_{2}$ directly.

One may point out that the appearance of a twofold topological degeneracy is not surprising at all, since the EF topological order may contain emergent fermions in the bulk with unit quantum dimension, whose condensation on a string only breaks the $Z_{2}^{f}$ symmetry [85]. Such a fermion condensed chain is nothing but the pSC chain [63].

Some strings in the fixed set may accidentally carry such a pSC chain. If one or three strings in the configuration in Fig. 19(a) carry a pSC chain, then the configuration will

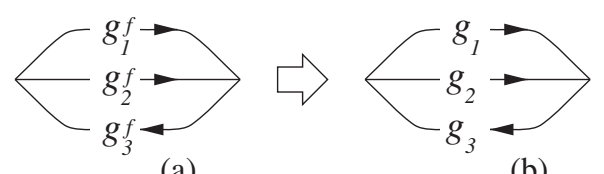

(a)

(b)

FIG. 19. (a) A string configuration in the bulk, described by the conjugacy class of a triple $\left(g_{1}^{f}, g_{2}^{f}, g_{3}^{f}\right)$ in $G_{f}$. (b) Moving to the boundary, the string configuration turns into one labeled by three group elements $\left(g_{1}, g_{2}, g_{3}\right)$ in $\hat{G}_{b}$. 
have a twofold topological degeneracy, coming from the two Majorana zero modes at the two junction points. So it seems that the appearance of a twofold topological degeneracy in the configurations in Fig. 19(a) is not a universal property. We can remove the two-fold topological degeneracy by choosing our fixed set of strings properly such that none of the strings in the fixed set carry a pSC chain. This choice indeed can be achieved when $m_{2}$ is a coboundary. However, when $m_{2}$ is a nontrivial cocycle, there is an obstruction in determining if a string carries a pSC chain or not. As a result, no matter how we choose the fixed set of strings, there are always some triples $g_{1}^{f}, g_{2}^{f}, g_{3}^{f}$ in the fixed set of strings, such that their configurations in Fig. 19(a) have twofold topological degeneracies.

How do we determine $m_{2}$ from the topological degeneracy of the configurations in Fig. 19(a)? We first measure the topological degeneracy in Fig. 19(a), where the three strings are chosen from the fixed set. If there is a twofold topological degeneracy, we assign

$$
m_{2}^{f}\left(g_{1}^{f}, g_{2}^{f}\right)=-1
$$

If there is no degeneracy, we assign

$$
m_{2}^{f}\left(g_{1}^{f}, g_{2}^{f}\right)=1 .
$$

From the function $m_{2}^{f}\left(g_{1}^{f}, g_{2}^{f}\right)$, we can determine the cohomology class of $m_{2} \in H^{2}\left(G_{b}, Z_{2}\right)$.

To see this, we first move the string configuration to the boundary. In this case, the bulk string labeled by $G_{f}$ becomes boundary local types labeled by $\hat{G}_{b}$. In other words, the bulk string types $g_{1}^{f}, g_{2}^{f}$, and $g_{3}^{f}$ in $G^{f}$ change to the boundary string types $g_{1}, g_{2}$, and $g_{3}$ in $\hat{G}_{b}$ [see Fig. 19(b)], which satisfy

$$
\pi^{f}\left(g_{i}^{f}\right)=\pi^{m}\left(g_{i}\right) \in G_{b},
$$

where $\pi^{f}$ and $\pi^{m}$ are the projections $G_{f} \stackrel{\pi^{f}}{\rightarrow} G_{b}$ and $\hat{G}_{b} \stackrel{\pi^{m}}{\rightarrow} G_{b}$, respectively.

Recall that the elements in $\hat{G}_{b}$ can be represented by $\left(g^{b}, x\right), g^{b} \in G_{b}$ and $x \in Z_{2}$. Thus, $g_{i}$ has a form $\left(g_{i}^{b}, x_{i}\right)$ where $g_{i}^{b}=\pi^{f}\left(g_{i}^{f}\right)$. For the strings in our fixed set, $x_{i}$ is also determined by $g_{i}^{f}$. Moreover, we can choose the fixed set properly such that the dependence of $x_{i}$ on $g_{i}^{b}=\pi^{f}\left(g_{i}^{f}\right)$ can be separated out. To see that, we first fix a choice of trapping potential for the $Z_{2}^{f}$ string $z$. Then, it is always possible to make the choice of $g^{f}, g^{f} z$ compatible with fusion with $z$, by first choosing the trapping potential for one of $g^{f}, g^{f} z$ and then choosing the trapping potential for the other as the fusion of the previous one with that of $z$, which we fix at the beginning. What is important for such choices is that the difference between $g^{f}$ and $g^{f} z$ is fixed by the beginning choice of $z$. We define $x_{z}=-1$ if our beginning choice of $z$ carries a pSC chain and $x_{z}=1$ otherwise. Then, for the above choices of the fixed set, we have

$$
x_{i}\left(g_{i}^{f} z\right)=x_{i}\left(g_{i}^{f}\right) x_{z} .
$$

Although the bulk string types satisfy $g_{1}^{f} g_{2}^{f}=g_{3}^{f}$, which leads to $g_{1}^{b} g_{2}^{b}=g_{3}^{b}$, the boundary string types $g_{i}$, as a particular lifting from $G_{b}$ to $\hat{G}_{b}$, may not satisfy $g_{1} g_{2}=g_{3}$. In fact, we have

$$
\begin{aligned}
{\left[g_{1}^{b}, x\left(g_{1}^{f}\right)\right]\left[g_{2}^{b}, x\left(g_{2}^{f}\right)\right] } & =\left[g_{1}^{b} g_{2}^{b}, m_{2}\left(g_{1}^{b}, g_{2}^{b}\right) x\left(g_{1}^{f}\right) x\left(g_{2}^{f}\right)\right] \\
& =\left[g_{1}^{b} g_{2}^{b}, \tilde{m}_{2} x\left(g_{1}^{f} g_{2}^{f}\right)\right] \\
& =\left(1, \tilde{m}_{2}\right)\left[g_{3}^{b}, x\left(g_{3}^{f}\right)\right]
\end{aligned}
$$

where

$$
\tilde{m}_{2}=m_{2}\left(g_{1}^{b}, g_{2}^{b}\right) \frac{x\left(g_{1}^{f}\right) x\left(g_{2}^{f}\right)}{x\left(g_{1}^{f} g_{2}^{f}\right)} .
$$

Using Eq. (59), it is not hard to see that $\left[x\left(g_{1}^{f}\right) x\left(g_{2}^{f}\right)\right] /$ $\left[x\left(g_{1}^{f} g_{2}^{f}\right)\right]$ depends only on $g_{i}^{b}=\pi^{f}\left(g_{i}^{f}\right)$ (since $x_{z}$ 's always appear in pairs) and is a coboundary on $G_{b}$. Thus, the above is, in fact, a 2-cocycle $\tilde{m}_{2}\left(g_{1}^{b}, g_{2}^{b}\right)$ on $G_{b}$ that is cohomologically equivalent to $m_{2}$. When $\tilde{m}_{2}\left[\pi^{f}\left(g_{1}^{f}\right), \pi^{f}\left(g_{2}^{f}\right)\right]=-1$, we have $g_{1} g_{2}=m g_{3}$ and the junction point carries a Majorana zero mode. In other words, the boundary configuration in Fig. 19(b) has a twofold topological degeneracy if $\tilde{m}_{2}\left[\pi^{f}\left(g_{1}^{f}\right), \pi^{f}\left(g_{2}^{f}\right)\right]=-1$.

Since the boundary configuration in Fig. 19(b) can be a short distance away from the boundary, moving to the boundary represents a weak perturbation. In this case, the boundary configuration in Fig. 19(b) having a twofold degeneracy implies that the corresponding bulk configuration in Fig. 19(a) also has a twofold degeneracy. In other words,

$$
\tilde{m}_{2}\left[\pi^{f}\left(g_{1}^{f}\right), \pi^{f}\left(g_{2}^{f}\right)\right]=m_{2}^{f}\left(g_{1}^{f}, g_{2}^{f}\right) .
$$

We see that the cocycle $\tilde{m}_{2}$ can be determined by measuring the topological degeneracy for bulk string configurations in Fig. 19(a). We note that $\tilde{m}_{2}$ and $m_{2}$ differ by a coboundary [Eq. (61)]. Thus, up to a coboundary, $m_{2}$ can be determined by measuring the topological degeneracy for bulk string configurations in Fig. 19(a).

We point out that, even when $G_{f}$ is non-Abelian, a nontrivial $Z_{2}^{m}$ extension, or $m_{2}$, still gives rise to the Majorana zero modes for some triple-string junctions. But in this case, there are extra topological degeneracies on junctions of three strings coming from the nonAbelianness of $G_{f}$. The appearance of topological 
degeneracy does not directly imply the appearance of Majorana zero modes. It is slightly more complicated to separate which topological degeneracy comes from nonAbelian $G_{f}$ and which comes from Majorana zero modes, which we describe in the following.

Again, the key step is to choose a fixed set of trapping potentials that trap strings labeled by $\chi_{g^{f}} \subset G_{f}$. Here, $\chi_{g^{f}}$ is the conjugacy class that contains $g^{f} \in G_{f}$. We stress that the different strings in the set can all be distinguished by their different braiding properties with the pointlike excitations. We call two strings equivalent if they have the same braiding properties with all the pointlike excitations. Thus, the strings in our fixed set are all inequivalent. We also assume our fixed set is complete, in the sense that it contains all inequivalent strings. In other words, the number of strings in the set is equal to the number of conjugacy classes in $G_{f}$.

We note that the condensation of the pointlike excitation can also form a stringlike excitation. For example, the condensation of $Z_{2}$ charges along a chain in a $Z_{2}$ gauge theory can form a stringlike excitation that have trivial braiding with all the pointlike excitations. We call such a kind of stringlike excitations descendant stringlike excitations, which are all equivalent to the trivial string. The above $Z_{2}$ charge condensed chain has a twofold degeneracy, since it is like a $Z_{2}$ symmetry-breaking state. As a result, the corresponding descendant stringlike excitation has quantum dimension 2. Our fixed set of strings does not contain strings that differ only by attaching a descendant stringlike excitation, since they are equivalent under braiding with pointlike excitations.

But each string in the fixed set may carry some additional descendant stringlike excitations. We reduce this ambiguity by requiring the strings in the fixed set do not carry descendant strings. This requirement is achieved by replacing each string in the set by its equivalent string with a minimal quantum dimension. However, this replacement still does not remove all the ambiguity due to a possible pSC chain attachment. Clearly, the pSC chain is a descendant string. But amazingly, despite the $Z_{2}^{f}$ symmetry breaking on an open pSC chain which leads to a twofold degeneracy, a closed pSC chain has no ground-state degeneracy. Thus, the pSC chain has quantum dimension 1. Attaching a pSC chain to a string will not change the quantum dimension of the string. So the strings in our fixed set, even after minimizing the quantum dimensions, may still carry pSC chains.

To test if the strings in our fixed set carry pSC chains or not, we choose three strings from our fixed set to form the configuration in Fig. 1. The topological degeneracy of the configuration is calculated in the following way. We first consider a set of pairs that have the form $\left(\tilde{g}_{1}, \tilde{g}_{2}\right)$, where $\tilde{g}_{1} \in \chi_{g_{1}^{f}}$ and $\tilde{g}_{2} \in \chi_{g_{2}^{f}}$. The two pairs $\left(\tilde{g}_{1}, \tilde{g}_{2}\right)$ and $\left(\tilde{g}_{1}^{\prime}, \tilde{g}_{2}^{\prime}\right)$ are equivalent if they are related by

$$
\tilde{g}_{1}^{\prime}=h \tilde{g}_{1} h^{-1}, \quad \tilde{g}_{2}^{\prime}=h \tilde{g}_{2} h^{-1}, \quad h \in G_{f} .
$$

The number of equivalence classes of the pairs, $N\left(\chi_{g_{1}^{f}}, \chi_{g_{2}^{f}}\right)$, is the topological degeneracy of the configuration in Fig. 1, provided that the three strings do not carry pSC chains. If one or three strings carry pSC chains, the topological degeneracy of the configuration in Fig. 1 will be given by $2 N\left(\chi_{g_{1}^{f}}, \chi_{g_{2}^{f}}\right)$. In this case, we say that the triplestring junction in Fig. 1 carries a Majorana zero mode.

Similar to the Abelian $G_{f}$ case, we assign $m_{2}^{f}\left(g_{1}^{f}, g_{2}^{f}\right)=1$ if the topological degeneracy of the configuration in Fig. 1 is $N\left(\chi_{g_{1}^{f}}, \chi_{g_{2}^{f}}\right)$ and $m_{2}^{f}\left(g_{1}^{f}, g_{2}^{f}\right)=-1$ if the topological degeneracy is $2 N\left(\chi_{g_{1}^{f}}, \chi_{g_{2}^{f}}\right)$. Clearly, $m_{2}^{f}$ satisfies

$m_{2}^{f}\left(g_{1}^{f}, g_{2}^{f}\right)=m_{2}^{f}\left(h_{1} g_{1}^{f} h_{1}^{-1}, h_{2} g_{2}^{f} h_{2}^{-1}\right), \quad h_{1}, h_{2} \in G_{f}$.

$m_{2}^{f}$ in the above is a cocycle in $Z^{2}\left(G_{f}, Z_{2}\right)$. Again, we can make $m_{2}^{f}\left(g_{1}^{f}, g_{2}^{f}\right)$ a function on $G_{b}$, by choosing the fixed set of strings properly, because, even for non-Abelian $G^{f}$, the $Z_{2}^{f}$ flux string $z$ is still invertible as $Z_{2}^{f}$ centralize $G^{f}$. Thus, we can still fix the choice of $z$ first and make the rest choices of the fixed set of strings compatible with the fusion with $z$. Now, if $g_{1}^{f}$ or $g_{2}^{f}$ is attached to an additional $z$ string, the total configuration in Fig. 1 always changes by fusion with a closed $z$ loop. As our choice of $z$ is fixed at the beginning, a closed $z$ loop does not carry any open pSC chain and, thus, introduces no additional degeneracy. Therefore, similar to the case of Abelian $G^{f}$, for nonAbelian $G^{f}, m_{2}^{f}$ also has the form

$$
m_{2}^{f}\left(g_{1}^{f}, g_{2}^{f}\right)=\tilde{m}_{2}\left[\pi^{f}\left(g_{1}^{f}\right), \pi^{f}\left(g_{2}^{f}\right)\right],
$$

where $\tilde{m}_{2} \in Z^{2}\left(G_{b}, Z_{2}\right)$. Again, such $\tilde{m}_{2}$ is equivalent to $m_{2}$ on the boundary. To understand such a result, consider moving the string configuration in Fig. 1 towards the canonical boundary. The string type changes from the bulk type $\chi_{g^{f}}$ to the boundary local type $g \in \hat{G}_{b}: \chi_{g^{f}} \rightarrow g$ that satisfy

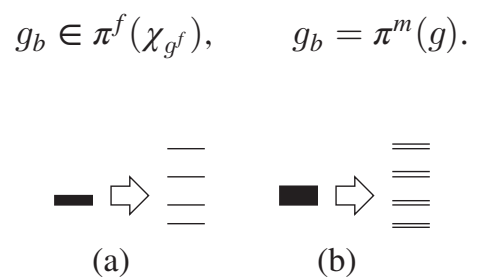

FIG. 20. The splitting of the topological degeneracy as we move string configuration Fig. 1 to wards the canonical boundary. (a) the case for topological degeneracy $N\left(\chi_{g_{1}^{f}}, \chi_{g_{2}^{f}}\right)$. (b) the case for topological degeneracy $2 N\left(\chi_{g_{1}^{f}}, \chi_{g_{2}^{f}}\right)$. 
The $N\left(\chi_{g_{1}^{f}}, \chi_{g_{2}^{f}}\right)$-fold or $2 N\left(\chi_{g_{1}^{f}}, \chi_{g_{2}^{f}}\right)$-fold topological degeneracy splits (see Fig. 20). However, only the degeneracy coming from conjugacy classes splits; the twofold topological degeneracy from Majorana zero modes is not affected by moving to the boundary. Each state [Fig. 20(a)] or twofold degeneracy [Fig. 20(b)] corresponds to the boundary configuration in Fig. 19(b). Hence, we obtain $\tilde{m}_{2}\left(g_{1}^{b}, g_{2}^{b}\right)$, which determines the $Z_{2}^{m}$ extension $\hat{G}_{b}$ of $G_{b}$ on the canonical boundary. If $\tilde{m}_{2}$ is a coboundary, we can choose a fixed set of strings such that all the triple-string junctions do not carry Majorana zero modes. The corresponding bulk topological order is an EF1 topological order. If $\tilde{m}_{2}$ is a nontrivial cocycle, then for any choice of a fixed set of strings, there are always triple-string junctions that carry Majorana zero modes. The corresponding bulk topological order is an EF2 topological order.

\section{F. Two necessary conditions for EF2 topological order}

From the bulk consideration in the last section, we see that the $m_{2}$ characterizing the EF2 topological orders are highly restricted. We focus on the particular $\tilde{m}_{2}$ that directly comes from measuring the Majorana zero modes in the bulk; it can differ from $m_{2}$ by a coboundary. First, the pullback of $\tilde{m}_{2}$ by $G_{f} \stackrel{\pi^{f}}{\rightarrow} G_{b}$ gives us $m_{2}^{f}=\left(\pi^{f}\right)^{*} \tilde{m}_{2} \in$ $H^{2}\left(G_{f}, Z_{2}\right)$ [see Eq. (65)]. Such a pullback must satisfy Eq. (64), which gives a condition on $\tilde{m}_{2}$ :

$$
\tilde{m}_{2}\left(g_{1}^{b}, g_{2}^{b}\right)=\tilde{m}_{2}\left(h_{1} g_{1}^{b} h_{1}^{-1}, h_{2} g_{2}^{b} h_{2}^{-1}\right), \quad h_{1}, h_{2} \in G_{b} .
$$

In other words, EF2 topological order can exist only when $G_{b}$ has nontrivial 2-cocycles with the above symmetry condition. This is the first necessary condition for EF2 topological orders. We note that, when $G_{b}$ is Abelian, the above condition becomes trivial and imposes no constraint.

We also point out that a $\mathrm{pSC}$ chain can be attached to a bulk string characterized by the conjugacy class $\chi_{g}$ of $G_{f}$ only when the centralizer group $Z_{g}\left(G_{f}\right)$ is a trivial $Z_{2}^{f}$ extension. Here, $Z_{g}\left(G_{f}\right)$ is the subgroup that commutes with an element $g$ in the conjugacy class $\chi_{g}$ :

$$
Z_{g}\left(G_{f}\right)=\left\{x \in G_{f} \mid g x=x g\right\} .
$$

Physically, the bulk string $\chi_{g}$ breaks the "symmetry" of the particles from $G_{f}$ down to $Z_{g}\left(G_{f}\right)$. If $Z_{g}\left(G_{f}\right)$ is not a trivial $Z_{2}^{f}$ extension, then a fermion condensation that breaks the $Z_{2}^{f}$ symmetry must also break some additional symmetries. In this case, we cannot attach a pSC chain to the bulk string $\chi_{g}$, since the pSC chain corresponds to a fermion condensation that breaks only the $Z_{2}^{f}$ symmetry [85].
Let us introduce an $M$ function on $G_{f}$ :

$$
M(g)= \begin{cases}0, & Z_{g}\left(G_{f}\right) \text { is a trivial } Z_{2}^{f} \text { extension } \\ 1, & \text { otherwise }\end{cases}
$$

Since

$$
Z_{g}\left(G_{f}\right)=Z_{z g}\left(G_{f}\right)
$$

where $z$ is the generator of $Z_{2}^{f}$, we have

$$
M(g)=M(z g)
$$

Therefore, we may also view $M$ as a function on $G_{b}$.

Since the bulk string $\chi_{g}, g \in G_{f}$, has no ambiguity of a pSC chain when $M(g)=1$, we see that $m_{2}^{f}$ satisfies

$$
m_{2}^{f}\left(g_{1}^{f}, g_{2}^{f}\right)=1, \quad \text { if } M\left(g_{1}^{f}\right)=M\left(g_{2}^{f}\right)=M\left(g_{1}^{f} g_{2}^{f}\right)=1 .
$$

This result becomes a condition on the cocycle $\tilde{m}_{2}$ on $G_{b}$ :

$\tilde{m}_{2}\left(g_{1}^{b}, g_{2}^{b}\right)=1, \quad$ if $M\left(g_{1}^{b}\right)=M\left(g_{2}^{b}\right)=M\left(g_{1}^{b} g_{2}^{b}\right)=1$.

This is the second necessary condition for EF2 topological orders. We note that the two conditions (67) and (73) are not invariant under adding coboundaries. Physically, on the canonical boundary, unlike in the bulk, since the $G_{f}$ symmetry is broken down to $Z_{2}^{f}$, it is always possible to attach pSC chains to strings, which can change $m_{2}$ by arbitrary coboundaries. Thus, generic $m_{2}$ may not satisfy Eqs. (67) and (73); we require Eqs. (67) and (73) only for a particular $\tilde{m}_{2}$ that is cohomologically equivalent to generic $m_{2}$.

As an example, for $G_{f}=Z_{4}^{f} \times G_{b}^{\prime}$, we find $M(g)=1$ for all $g \in Z_{4}^{f} \times G_{b}^{\prime}$. Thus, there is no EF2 topological order with $G_{f}=Z_{4}^{f} \times G_{b}^{\prime}$.

\section{A GENERAL FRAMEWORK FOR 3 + 1D TOPOLOGICAL ORDERS WITH SYMMETRIES}

We see that in $3+1 \mathrm{D}$ the intrinsic topological orders are closely related to SPT phases. In the above section, we show that the data and conditions classifying EF topological orders have the same form as those classifying fermionic SPT phases. Without the pSC chain, both EF1 topological orders and fermionic SPT phases are classified by the group supercohomology theory; with the pSC chain, also very strong evidence indicates that the classifying data and conditions have the same form. Combined with our previous results on $3+1 \mathrm{D} A B$ topological orders, we 
conclude that all $3+1 \mathrm{D}$ topological orders correspond to gauged $3+1 \mathrm{D}$ SPT phases: AB topological orders correspond to gauged bosonic SPTs, and EF topological orders correspond to gauged fermionic SPTs.

Our classification and construction of EF topological orders also leads to a classification and construction of $3+1 \mathrm{D}$ fermionic SPT orders: A $3+1 \mathrm{D}$ fermionic SPT order has a unique canonical boundary that breaks the fermion symmetry $G_{f}$ down to $Z_{2}^{f}$. Such a symmetrybreaking boundary contains strings (i.e., domain walls between different symmetry-breaking group states). It also contains a fermionic pointlike excitation. Because of such a boundary fermion, the strings (the domain walls) on the boundary are labeled by elements $g \in \hat{G}_{b}=Z_{2}^{m} \lambda G_{b}$. The elements in $G_{b}$ describe the symmetry transformation that change one symmetry-breaking ground state to the other symmetry-breaking ground state across the domain wall. The elements in $Z_{2}^{m}=\{\mathbf{1}, m\}$ describe if the boundary string carries the pSC chain formed by the boundary fermions. In fact, we know only if two strings differ by a pSC chain or not. We may not be able to determine which string carries the $\mathrm{pSC}$ chain and which string does not. This limitation leads to string labels $\hat{G}_{b}=Z_{2}^{m} \lambda G_{b}$ rather than $Z_{2}^{m} \times G_{b}$. At a triple-string junction, we may have an end of a pSC chain. Such a triple-string junction will carry an Majorana zero mode. The above picture leads to a classification and a construction of $3+1 \mathrm{D}$ fermionic SPT order using the data of EF 2-category $\mathcal{A}_{b}^{3}$.

The SPT and the topological order are the end points of ungauging and gauging procedures, respectively. They are also the two extreme cases with only symmetry no intrinsic topological order and only intrinsic topological order no symmetry. Because of these, it is natural to conjecture that if we partially gauge a SPT or ungauge a topological order, in between we should get a state with both symmetry and topological order, in other words, a SET. Therefore, we expect the following general classification framework for $3+1 \mathrm{D}$ topological phases with symmetries:

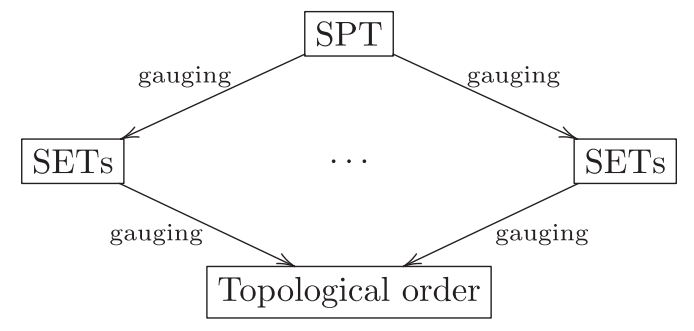

Different partially gauging procedures, equivalently different subgroup sequences $H_{1} \subset H_{2} \subset \cdots \subset G$, give rise to different sequences of intermediate SETs. The starting point, SPT, and end point, topological order, are fixed. They share the same classification data, according to our results. We believe that in the same gauging sequence the phases also share the same classification data as the starting
SPT and the ending topological order. However, physical interpretations and equivalence relations of the same classification data are different at different steps.

In particular, fermionic SETs and topological orders (note that EF topological order is a bosonic topological order with emergent fermionic particles) should be special cases starting from fermionic SPTs but keep the fermion number parity, namely, $Z_{2}^{f}$, not gauged until the last step:

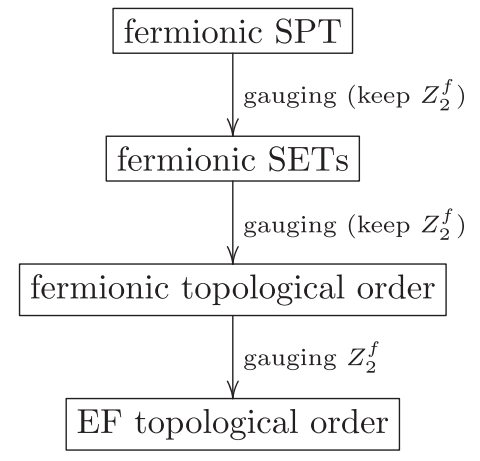

Recall that in $2+1 \mathrm{D}$ we classify topological phases with symmetry by a triple of categories $\mathcal{E} \subset \mathcal{C} \subset \mathcal{M}[86,87]$, where $\mathcal{E}$ is the symmetric category of local excitations and corresponds to the representations of the symmetry group, $\mathcal{E}=\mathcal{R e p}(G)$ or $\mathcal{E}=\operatorname{s} \mathcal{R} \operatorname{ep}\left(G_{f}\right), \mathcal{C}$ is the category of all bulk excitation, and $\mathcal{M}$ is the gauged theory. In particular, for $2+1 \mathrm{D}$ SPT phases, we have $\mathcal{E}=\mathcal{C} \subset \mathcal{M}$. Now this idea naturally generalizes to $3+1 \mathrm{D}$, since any $3+1 \mathrm{D}$ topological order contains a symmetric subcategory $\mathcal{E}$ corresponding to its pointlike excitations and can be viewed as a gauged SPT $\mathcal{M}$ with original global symmetry $\mathcal{E}$. A generic $3+1 \mathrm{D}$ SET with global symmetry $\mathcal{E}$ is then described by a certain 2-category $\mathcal{C}$ satisfying $\mathcal{E} \subset \mathcal{C} \subset \mathcal{M}$. In the gauging procedures, the modular extension $\mathcal{M}$ remains the same (which is why we say that phases in the same gauging sequence share the same classification data), while $\mathcal{E}$ and $\mathcal{C}$ become smaller and larger, respectively, which can be understood as part of global symmetry $\mathcal{E}$ "gauged" into gauge symmetry $\mathcal{C}$. For example, $\mathcal{E}=\mathcal{C}=\mathcal{R e p}(G)$ or $\operatorname{s} \mathcal{R e p}\left(G_{f}\right)$ for the SPT phase, while $\mathcal{E}$ is trivial and $\mathcal{C}=\mathcal{M}$ for the topological order.

As we already have a good understanding about the $3+1 \mathrm{D}$ SPT phases, it is thus quite hopeful for a complete understanding of $3+1 \mathrm{D}$ topological orders and symmetries by thoroughly studying the (partially) gauging procedures.

\section{ACKNOWLEDGMENTS}

We thank Zheng-Cheng Gu for helpful discussions and Chenjie Wang for sharing his unpublished result about the appearance of Majorana zero modes on linked loops in some $3+1 \mathrm{D}$ fermionic SPT states. X.-G. W. is supported by National Science Foundation Grants No. DMR1506475 and No. DMS-1664412. Part of the work was 
done at Perimeter Institute for Theoretical Physics and Tsinghua University. Research at Perimeter Institute is supported by the Government of Canada through Industry Canada and by the Province of Ontario through the Ministry of Research. T.L.'s visit at Tsinghua University was supported by Chinese Ministry of Education under Grant No. 20173080024.

\section{APPENDIX A: TANNAKA DUALITY}

Our approach in this paper relies heavily on the Tannaka duality [88], or Tannaka reconstruction theorem for group representations. In this Appendix, we give a physical introduction of the Tannaka duality. In the meantime, we also introduce and explain some important concepts used in this paper in detail.

\section{Two physical models}

A physical motivation of the Tannaka duality is the following: Let us consider a bosonic or a fermionic system with a symmetry $G$. We assume the ground state to be a product state that does not break the symmetry. If we only measure the system via probes that do not break the symmetry, can we detect the symmetry group of the system? We note that a symmetry transformation acting on the system is not observable, unless the system actually changes in certain ways to reflect that some action happened. Thus, we need to break the symmetry in order to measure the symmetry transformation directly. In contrast, the symmetric probes produce only objects that do not break the symmetry, such as particles trapped by the symmetric potential that are described by representations $\rho$ of the symmetry group: $\rho \in \operatorname{Rep}(G)$. On the other hand, the symmetric probes do allow us to fuse and braid those symmetric particles in arbitrary ways.

To describe those fusion and braiding processes, the concept of fusion space is important: If the particles are obtained by symmetric trap potentials, then the fusion space $\mathcal{V}$ is simply the ground-state subspace of the total Hamiltonian with traps: $H_{\text {tot }}=H_{0}+\sum_{i} \Delta H_{\text {trap }}\left(x_{i}\right)$ which trap particles $p_{i}$ at $x_{i}$. We denote the fusion space by $\mathcal{V}\left(M, p_{1}, p_{2}, \ldots\right)$, where $M$ is the space manifold that supports our system. So the fusion and the braiding processes, as well as the symmetric deformation of the Hamiltonians $H_{0}$ and $\Delta H_{\text {trap }}$, correspond to unitary linear maps on the fusion space. The Tannaka duality tells us how to use those symmetric operations, i.e., the linear maps on the fusion space $\mathcal{V}\left(M, p_{1}, p_{2}, \ldots\right)$, to obtain the symmetry group $G$.

Mathematically, the fusion and braiding, as well as the symmetric deformation of the Hamiltonians $H_{0}$ and $\Delta H_{\text {trap }}$, on all the possible trapped particles form a structure which is denoted by $\mathcal{R e p}(G)$ if all the particles are bosons or by $\operatorname{R} \operatorname{ep}(G)$ if some particles are fermions. Such a structure is called a symmetric fusion category. The particles are labeled by the representations of $G$, which form a set $\operatorname{Rep}(G)$. So a symmetric fusion category $\mathcal{R e p}(G)$ or $\operatorname{s} \operatorname{Rep}(G)$ contains the set $\operatorname{Rep}(G)$ whose elements are called objects (which correspond to trapped particles). $\mathcal{R e p}(G)$ or $\operatorname{s} \mathcal{R} \operatorname{ep}(G)$ also contains addition data that describe fusion and braiding of particles in $\operatorname{Rep}(G)$. In particular, the fusion of the particles is nontrivial, since the particles are described by the representations of $G$, and the fusion of the representations is nontrivial.

If we know just the set of representations $\operatorname{Rep}(G)$, we cannot recover the group $G$. But if we also know all symmetric operations, such as fusion and braiding, as well as the symmetric deformation of the Hamiltonians $H_{0}$ and $\Delta H_{\text {trap }}$, in other words, if we know $\mathcal{R e p}(G)$ or s $\mathcal{R e p}(G)$, then according to the Tannaka duality, we can recover the group $G$.

Another physical motivation of the Tannaka duality is more relevant to this paper. We consider a $3+1 \mathrm{D}$ topological order $\mathcal{C}^{4}$. The pointlike excitations in the topological order are bosons or fermions with trivial mutual statistics. Those particles have a nontrivial fusion rule. The fusion and braiding of those particles are also described by a symmetric fusion category $\mathcal{E}$. The Tannaka duality tells us that, from $\mathcal{E}$, we can recover a group $G$. Thus, each $3+1 \mathrm{D}$ topological order contains a hidden group $G$. In this second example, we do not even have a symmetry. All the operations, such as fusion, braiding, and deformation of $H_{0}$ and $\Delta H_{\text {trap }}$, are allowed, as long as they are generated by local interaction. But how can one recover a group from a problem that has no symmetry?

In the first example, we do have symmetry, but we want to recover the symmetry group via the symmetric operations. In the second example, we want to recover the hidden group in $3+1 \mathrm{D}$ topological order which has no symmetry. These two problems happen to be the same problem, which is solved by the Tannaka duality.

\section{Tannaka duality I: All boson \\ a. Statement of Tannaka duality}

For the moment, we restrict to an all-boson symmetric fusion category $\mathcal{E}$. Mathematically, the Tannaka duality states that we can reconstruct a group $G$ from symmetric fusion category $\mathcal{E}$ by the automorphisms of a fiber functor, namely, a braided monoidal functor $F$, from $\mathcal{E}$ to the category of vector spaces, $\mathcal{V e c}$ :

$$
G \equiv \operatorname{Aut}(F: \mathcal{E} \rightarrow \mathcal{V e c}),
$$

and the Tannaka duality tells us that

$$
\mathcal{E} \cong \mathcal{R e p}(G)
$$

This process is how we find the hidden group in a symmetric fusion category $\mathcal{E}$. 
To understand the Tannaka duality, let us choose the symmetric fusion category to be the category formed by the representations of a finite group $\operatorname{Rep}(G)$. We want to find out, what are the automorphisms of a fiber functor $\operatorname{Aut}[F: \mathcal{R e p}(G) \rightarrow \mathcal{V e c}]$ ?

Let us first describe the representation category $\mathcal{R e p}(G)$.

(1) An object in $\operatorname{Rep}(G)$ is a group representation $p$, which corresponds to a pair $p \equiv\left[V(p), \rho_{p}\right]$, where $V(p)$ is a vector space equipped with a group action $\rho_{p}: G \rightarrow \mathrm{GL}[V(p)]$. The set of objects in $\operatorname{Rep}(G)$ consists of all such pairs.

(2) The morphisms in the symmetric fusion category $\mathcal{R e p}(G), p^{\prime} \rightarrow p$, correspond to the linear map $u: V\left(p^{\prime}\right) \rightarrow V(p)$ which commutes with the group action, $\rho_{p}(g) u=u \rho_{p^{\prime}}(g)$. The morphisms allow us to define the notion of simple objects which correspond to irreducible representations.

(3) Representations can be "fused" $p_{1} \otimes p_{2}$, which corresponds to taking the tensor product of the vector spaces $V\left(p_{1}\right) \otimes_{\mathbb{C}} V\left(p_{2}\right)$ and the new group action is $\rho_{p_{1} \otimes p_{2}}(g)=\rho_{p_{1}}(g) \otimes_{\mathbb{C}} \rho_{p_{2}}(g)$ :

$$
p_{1} \otimes p_{2}=\left[V\left(p_{1}\right) \otimes_{\mathbb{C}} V\left(p_{2}\right), \rho_{p_{1}}(g) \otimes_{\mathbb{C}} \rho_{p_{2}}(g)\right] .
$$

In this case, we have the forgetful functor that maps a representation category $\mathcal{R e p}(G)$ to the category of vector spaces $\mathcal{V e c}, F: p \equiv\left[V(p), \rho_{p}\right] \mapsto V(p)$ (forgetting the group action part), which is called a fiber functor. An automorphism of such a fiber functor $F$ is a set of unitary maps, $\alpha=\left\{\alpha_{p}: V(p) \rightarrow V(p)\right\}$, one map for each $p$. Such a set of maps must be compatible with the fusion rule described above, as well as the morphisms $p^{\prime} \rightarrow p$ : $V\left(p^{\prime}\right) \stackrel{u}{\rightarrow} V(p)$, i.e., satisfying $\alpha_{p} u=u \alpha_{p^{\prime}}$. The set of all those automorphisms forms a group

$$
\alpha \cdot \alpha^{\prime}=\left\{\alpha_{p}\right\} \cdot\left\{\alpha_{p}^{\prime}\right\}=\left\{\alpha_{p} \alpha_{p}^{\prime}\right\}
$$

Such a group is the automorphism group, which happens to be $G$ :

$$
G \cong \operatorname{Aut}[F: \mathcal{R e p}(G) \rightarrow \mathcal{V e c}]
$$

This is because, to be compatible with the morphisms and the fusion rule, $\alpha_{p}$ has to be $\rho_{p}(h)$ for a certain $h \in G$. In fact, this is how we recover the symmetry group $G$ in the first model.

In the following, we describe Tannaka's construction and the above statements, in terms of the two physical models described above, where the particles are described by a symmetric fusion category $\mathcal{E}$. This way, one may gain a more physical understanding of the Tannaka duality.

\section{b. Irreducible representations from symmetry operations}

Before trying to obtain the group, let us try to obtain the irreducible representations of the group first. In general, a particle $p \in \mathcal{E}$ (trapped by a symmetric potential in the first model) corresponds to a representation. But which particles correspond to irreducible representations? To address this question, we start with the fusion space of $p$ with other particles $\mathcal{V}(M, p, q, \ldots)$. Note that $\mathcal{V}(M, p, q, \ldots)$ is the ground-state subspace of $H_{0}+\Delta H_{\text {trap }}\left(x_{p}\right)+\Delta H_{\text {trap }}\left(x_{q}\right)+$ $\cdots$ that traps the particle $p$ at $x_{p}$, particle $q$ at $x_{q}$, etc. By deforming (or deforming while preserving the symmetry for the first model) just $\Delta H_{\text {trap }}\left(x_{p}\right)$ to $\Delta H_{\text {trap }}^{\prime}\left(x_{p}\right)$, we may split the ground-state degeneracy

$$
\mathcal{V}(M, p, q, \ldots)=\mathcal{V}_{1} \oplus \mathcal{V}_{2} \oplus \cdots
$$

The new ground-state subspace $\mathcal{V}_{1}$ can be viewed as the fusion space of another particle $p^{\prime}$ at $x_{p}$ with other particles $q, \ldots, \mathcal{V}_{1}=\mathcal{V}\left(M, p^{\prime}, q, \ldots\right)$. Thus, the above splitting of $\mathcal{V}(M, p, q, \ldots)$ can be rewritten as

$$
\mathcal{V}(M, p, q, \ldots)=\mathcal{V}\left(M, p^{\prime}, q, \ldots\right) \oplus \mathcal{V}_{2} \oplus \cdots
$$

Then, we say that there is a morphism from $p^{\prime}$ to $p: p^{\prime} \rightarrow p$ [89]. Here, a morphism $p^{\prime} \rightarrow p$ can be understood as that the fusion space of $p^{\prime}$, after a proper unitary transformation, is contained in the fusion space of $p$. If we have morphisms in both directions $p^{\prime} \rightarrow p$ and $p \rightarrow p^{\prime}$, then the fusion space of $p$ is the same as the fusion space of $p^{\prime}$, up to an unitary transformation. If $p^{\prime} \rightarrow p$ implies $p \rightarrow p^{\prime}$, for all $p^{\prime}$ 's, then the fusion space of $p$ is minimal. For the case of the first model, it means that $p$ corresponds to an irreducible representation of the symmetry group. For the second model, we can formally regard $p$ as an irreducible representation of some group $G$. In the category theory, we call such a minimal $p$ a simple object. In this paper, we also call $p$ a simple particle.

There is always a trivial simple particle denoted by $\mathbf{1}$. It corresponds to local excitations that can be created by local symmetric operators in the first model or local operators in the second model. Its fusion space has a property

$$
\mathcal{V}(M, \mathbf{1}, p, q, \ldots) \cong \mathcal{V}(M, p, q, \ldots) .
$$

It is not hard to see that the full splitting of the fusion space is given by [see Eq. (A7)]

$\mathcal{V}(M, p, q, \ldots)=\mathcal{V}\left(M, p_{1}, q, \ldots\right) \oplus \mathcal{V}\left(M, p_{2}, q, \ldots\right) \cdots$.

In this case, we say the particle $p$ is a direct sum of particle $p_{1}, p_{2}$, etc.: 


$$
p=p_{1} \oplus p_{2} \oplus \cdots
$$

Physically, it means that the particle $p$ is an accidental degeneracy of particle $p_{1}$, particle $p_{2}$, etc. For example, in the first model, we may have a particle which is an accidental degeneracy of spin-up and spin-down particles. Such a degeneracy becomes required in the presence of $S U(2)$ spin rotation symmetry. In this case, a spin- $1 / 2$ particle is a simple particle (i.e., the fusion space cannot be split further). If we break the $S U(2)$ symmetry, then the spin-1/2 particle becomes a composite particle which is a direct sum of two simple particles, a spin-up and a spindown particle. For the case of the first model, we see that the symmetric operations of deforming $\Delta H_{\text {trap }}\left(x_{p}\right)$, which correspond to the morphisms in the category theory, allow us to define the notion of irreducible representation without using group transformation and other symmetry-breaking operations.

\section{c. Fusion rules of particles}

We may view two nearby simple particles $p_{1}$ and $p_{2}$ (i.e., two irreducible representations) as one particle $p_{3}$ (i.e., one representation):

$$
p_{1} \otimes p_{2}=p_{3} .
$$

In general, $p_{3}$ is no longer a simple particle (i.e., no longer an irreducible representation):

$$
p_{1} \otimes p_{2}=p_{3}=p_{1}^{\prime} \oplus p_{2}^{\prime} \oplus \cdots
$$

Sometimes, the particle types on the right may repeat:

$$
p_{1} \otimes p_{2}=p_{1}^{\prime} \oplus p_{1}^{\prime} \oplus p_{2}^{\prime} \oplus \cdots=2 p_{1}^{\prime} \oplus p_{2}^{\prime} \oplus \cdots .
$$

We may rewrite the above as

$$
p_{i} \otimes p_{j}=\bigoplus_{k} N_{k}^{i j} p_{k},
$$

which is called the fusion rule of the (simple) particles. From Eq. (A8), we see that the trivial particle $\mathbf{1}$ is the unit of the fusion operation:

$$
\mathbf{1} \otimes p=p \otimes \mathbf{1}=p
$$

Using $N_{k}^{i j}$, we can calculate dimension of the fusion space with $n$ particles $p_{i}$ on $S^{3}$, which has the form

$\operatorname{dim} \mathcal{V}\left(S^{3}, p_{i}, p_{i}, \ldots, p_{i}\right)=\operatorname{dim} \mathcal{V}\left(S^{3}, p_{i}^{\otimes n}\right) \sim d_{i}^{n}$

in the $n \rightarrow \infty$ limit. The number $d_{i}$ is called the quantum dimension of the $p_{i}$ particle. One can show that $d_{i}$ is the largest positive eigenvalue of matrix $N_{i}$, where the matrix elements of $N_{i}$ is given by $\left(N_{i}\right)_{k j}=N_{k}^{i j}$.

For the case of the first model, Eq. (A14) corresponds to the decomposition of the tensor product of irreducible representations. We see that additional information about the symmetry group $G$, the decomposition of the tensor product of irreducible representations, can also be obtained from symmetric operations: the fusion of particles (which is realized by bringing two symmetric traps together). From $N_{k}^{i j}$, we can even obtain the dimensions of irreducible representations $p_{i}$, which are given by the quantum dimensions $d_{i}$, which, in turn, determines the number of elements in the symmetry group $G$ :

$$
\sum_{i \text { is simple }} d_{i}^{2}=|G| .
$$

We get more information about the group without using any symmetry-breaking operations.

\section{d. Braiding and topological spin of particles}

Consider a fusion space $\mathcal{V}(M, p, q, \ldots)$. If we adiabatically exchange the two particles $p$ and $q$, the resulting fusion space $\mathcal{V}(M, q, p, \ldots)$ is always isomorphic to the original one, no matter what the manifold $M$ and background particles or strings are. Therefore, we say that there is a braiding morphism $c_{p, q}$ for the fusion $p \otimes q$ :

$$
c_{p, q}: p \otimes q \cong q \otimes p
$$

In general, we need to specify the exchange path (for example, clockwise or counterclockwise in $2+1 \mathrm{D}$ ). But for the above two physical models, braiding is, in fact, path independent, which is the defining property of the symmetric fusion category, that for all particles $p, q$,

$$
c_{q, p} c_{p, q}=\mathrm{id}_{p \otimes q} .
$$

This result means that braiding $p$ a whole loop around $q$ is the same as doing nothing, which is equivalent to path independence.

We can also extract the topological spin of simple particle $p$. Given a fusion space $\mathcal{V}(M, p, \ldots)$, we twist $p$ by $2 \pi$, and the fusion space then acquires a phase factor $\theta_{p}$, called the topological spin of $p$. It is, in fact, determined by the braiding $c_{p, p}$. In the case of the symmetric fusion category, $\theta_{p}$ helps to distinguish bosons and fermions:

$$
\theta_{p}= \begin{cases}1, & p \text { is a boson } \\ -1, & p \text { is a fermion }\end{cases}
$$




\section{e. Physical realization of fiber functor}

The Tannaka duality requires a fiber functor, which associates a vector space $F(p)$ to a particle $p$, such that it realizes the fusion and braidings of particles, in terms of the tensor product and the (trivial) braiding of vector spaces:

$$
\begin{aligned}
F(p \otimes q) & \cong F(p) \otimes_{\mathbb{C}} F(q), \\
F\left(c_{p, q}\right) & =c_{F(p), F(q)},
\end{aligned}
$$

as if $F(p)$ are local Hilbert spaces. Here, the braiding for vector spaces is the usual one:

$c_{V, W}: v \otimes_{\mathbb{C}} w \mapsto w \otimes_{\mathbb{C}} v, \quad \forall v \in V, w \in W$.

We note that if a functor preserves the fusion (it is a monoidal functor), whether preserving braiding or not is just a property of the monoidal functor, not an additional structure (like being an Abelian group or not is a property of a group).

We see a necessary condition for the fiber functor to exist is that particles are all bosons with trivial braiding. It turns out that it is also sufficient.

Physically, only the operations on the fusion spaces are measurable (or physical). So the question is, which fusion space should be associated to the particle $p$ in order to have a fiber functor? One might naturally choose the fusion space to be $\mathcal{V}\left(S^{3}, p\right)$ (i.e., the fusion space of a particle $p$ on the space of a 3 -sphere $S^{3}$ ). But $\mathcal{V}\left(S^{3}, p\right)=\varnothing$ for a nontrivial particle. So we need to add (nonsimple) background particles to make the fusion space nonzero for any added particles. The question is, what background particles should we insert besides $p$, to get a fusion space satisfying the conditions (A21)?

It turns out that we do have a special background (nonsimple) particle to achieve this goal. Let us denote it by $Q$, which has a direct sum decomposition in terms of the simple particles and their quantum dimensions $d_{i}$ :

$$
Q=\bigoplus_{i} d_{i} p_{i}
$$

The fusion space $\mathcal{V}\left(S^{3}, p, Q\right)$ satisfies

$\mathcal{V}\left(S^{3}, p \otimes q, Q\right) \cong \mathcal{V}\left(S^{3}, p, Q\right) \otimes_{\mathbb{C}} \mathcal{V}\left(S^{3}, q, Q\right)$.

[In the first example, $Q$ is nothing but the reducible representation $\operatorname{Fun}(G)$, all the functions on $G$. It is the regular representation of $G$.] Therefore, we can take

$$
F(p) \equiv \mathcal{V}\left(S^{3}, p, Q\right)
$$

It preserves fusion by Eq. (A24) and also braiding (its property but we do not show it explicitly here), thus a desired fiber functor.

\section{f. Automorphism of the fiber functor}

Now we have a fiber functor that maps every particle $p$ to a vector space $F(p)=\mathcal{V}\left(S^{3}, p, Q\right)$. Physically, the vector space $F(p)=\mathcal{V}\left(S^{3}, p, Q\right)$ is the ground-state subspace of a Hamiltonian on $S^{2}$ with two traps: $H_{0}+\Delta H_{p}+\Delta H_{Q}$, where $\Delta H_{Q}$ traps a particular composite particle $Q=\bigoplus_{i} d_{i} p_{i}$ (a particle with accidental degeneracy).

Next, we describe the automorphism of the fiber functor. An automorphism is a choice of an unitary map on $F(p)=$ $\mathcal{V}\left(S^{3}, p, Q\right)$ for each particle $p$. We denote those unitary maps by $\alpha_{p}$. So an automorphism corresponds to a set of unitary maps $\alpha \equiv\left\{\alpha_{p}\right\}$. But not every set of unitary maps, $\left\{\alpha_{p}\right\}$, is an automorphism. An automorphism also needs to preserve all the structures of the fiber functor and, as a result, needs to satisfy many conditions. But what are those conditions?

We explain that deforming the trap potential $\Delta H_{p}$ (while preserving the symmetry in the first model) may split that fusion space $\mathcal{V}\left(S^{3}, p, Q\right)=\mathcal{V}\left(S^{3}, p^{\prime}, Q\right) \oplus \cdots$. This splitting leads to a morphism $p^{\prime} \rightarrow p$. Under the fiber functor $F$ which takes a special fusion space, the morphism $p^{\prime} \rightarrow p$ gives rise to an embedding map $u: F\left(p^{\prime}\right) \rightarrow F(p)$. An automorphism $\alpha=\left\{\alpha_{p}\right\}$ must be compatible with all those embedding maps:

$$
u \alpha_{p^{\prime}}=\alpha_{p} u
$$

or

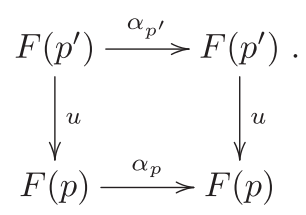

In the first model, $F(p)$ is, in general, a reducible representation of the symmetry group $G$. When $p^{\prime}$ is a simple particle, all embedding maps $u$ tell us all different ways to embed irreducible representation $F\left(p^{\prime}\right)$ into the reducible one $F(p)$. The condition (A27) tells us that $\alpha_{p}$ is block diagonal and fully determined by its components on different simple particles (irreducible representations) $\alpha_{p^{\prime}}$.

The automorphism $\alpha=\left\{\alpha_{p}\right\}$ also needs to be compatible with the fusion of particles. We may view two well-separated particles $p_{1}$ and $p_{2}$ as a single particle $p_{3}=p_{1} \otimes p_{2}$. The unitary maps $\alpha_{p_{1}}, \alpha_{p_{2}}$, and $\alpha_{p_{3}}$ should be related. Since the fusion space from the fiber functor satisfies Eq. (A21), we require that $\alpha_{p_{3}}$ equals the tensor product of $\alpha_{p_{1}}$ and $\alpha_{p_{2}}$ [up to the isomorphism fixed by the fiber functor (A21)]: 


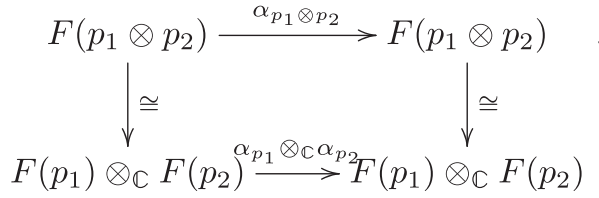

Since $p_{3}=p_{1} \otimes p_{2}=\bigoplus_{i} p_{i}^{\prime}$ and $F\left(p_{1} \otimes p_{2}\right) \cong \bigoplus_{i} F\left(p_{i}^{\prime}\right)$, the above can be rewritten as

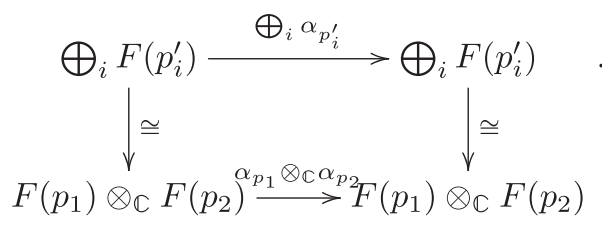

The above is the condition for the automorphism $\alpha=\left\{\alpha_{p}\right\}$ to be compatible with the fusion which is a data point in $\mathcal{R e p}(G)$.

The set of unitary maps $\alpha=\left\{\alpha_{p}\right\}$ that satisfies Eqs. (A27) and (A29) is called an automorphism of the fiber functor. If $\alpha=\left\{\alpha_{p}\right\}$ and $\alpha^{\prime}=\left\{\alpha_{p}^{\prime}\right\}$ are two automorphisms, we can show that $\alpha \cdot \alpha^{\prime} \equiv\left\{\alpha_{p} \alpha_{p}^{\prime}\right\}$ is also an automorphism. So the automorphisms form a group $G \equiv \operatorname{Aut}(F)$. Such a group corresponds to the symmetry group in the first physical model. We measure the symmetry group using only symmetric probes. In the second physical model, $G$ is a group associated with the $3+1 \mathrm{D}$ topological order. We show that every $3+1 \mathrm{D}$ topological order is associated with an unique group $G$.

To emphasize the group nature of the automorphisms $\alpha \equiv\left\{\alpha_{p}\right\}$, we may instead write $g \equiv\left\{g_{p}\right\} \in G \equiv \operatorname{Aut}(F)$. They give rise to the group action on $F(p)$, by $\rho_{p}(g)=g_{p}$.

\section{Example of Tannaka reconstruction for $\mathcal{R e p}\left(Z_{2}\right)$}

In this section, we illustrate the Tannaka duality with the simplest example, $\operatorname{Rep}\left(Z_{2}\right)$. We follow the general reconstruction procedure, trying to show the flavor of the abstract theorem.

First, let us describe $\operatorname{Rep}\left(Z_{2}\right)$ in terms of fusion. There are two irreducible representations of $Z_{2}$ : the trivial denoted by $\mathbf{1}$, the nontrivial one denoted by $e$. The fusion rule is

$$
1 \otimes \mathbf{1}=\mathbf{1}, \quad \mathbf{1} \otimes e=e \otimes \mathbf{1}=e, \quad e \otimes e=\mathbf{1} .
$$

The background charge is $Q=\mathbf{1} \oplus e$. We find that $F(e)=$ $\mathcal{V}\left(S^{3}, e \otimes Q\right)=\mathcal{V}\left(S^{3}, e \oplus \mathbf{1}\right)=\mathcal{V}\left(S^{3}, \mathbf{1}\right)=\mathcal{V}\left(S^{3}\right)=\mathbb{C}$. The ground state on $S^{3}$ is nondegenerate; thus, $F(e)$ is one dimensional. Similarly, $F(\mathbf{1})$ is one dimensional as well.

When $p$ is composite, $p=\bigoplus_{i} p_{i}$, Eq. (A27) tells us that $\alpha_{p}$ is block diagonal:

$$
\alpha_{p}=\bigoplus_{i} \alpha_{p_{i}}
$$

where $p_{i}$ are simple. Since $F\left(p_{i}\right)$ for a simple particle is always one dimensional for $\operatorname{Rep}\left(Z_{2}\right), \alpha_{1}$ and $\alpha_{e}$ are just phase factors. Equation (A29) requires that

$$
\alpha_{1 \otimes e}=\alpha_{1} \otimes_{\mathbb{C}} \alpha_{e}=\alpha_{e} .
$$

Thus, $\alpha_{1}=1$. Equation (A29) also requires that

$$
\alpha_{e \otimes e}=\alpha_{e} \otimes_{\mathbb{C}} \alpha_{e}=\alpha_{1}=1 .
$$

Thus, $\alpha_{e}= \pm 1$. The solution $\left\{\alpha_{1}=1, \alpha_{e}=1\right\}$ corresponds to an automorphism, and the solution $\left\{\alpha_{1}=1\right.$, $\left.\alpha_{e}=-1\right\}$ corresponds to the other automorphism. The composition $\left\{\alpha_{1}, \alpha_{e}\right\}\left\{\alpha_{1}^{\prime}, \alpha_{e}^{\prime}\right\}=\left\{\alpha_{1} \alpha_{1}^{\prime}, \alpha_{e} \alpha_{e}^{\prime}\right\}$ is the group multiplication, which tells us that $\left\{\alpha_{1}=1, \alpha_{e}=1\right\}$ and $\left\{\alpha_{1}=1, \alpha_{e}=-1\right\}$ do form a $Z_{2}$ group.

\section{Tannaka duality II: With fermions}

We proceed to introduce the Tannaka duality for symmetric fusion category $\mathcal{E}$ which contains fermions. Strictly speaking, the fermion or "super" case is due to Deligne's theorem [56], which generalized Tannaka's original idea.

The super case is almost the same: Find a fiber functor, calculate the automorphisms of the fiber functor, and recover the group. But the fiber functor needs to preserve braiding, while in $\mathcal{V}$ ec there are only bosons. So we have to change the target of the fiber functor to accommodate fermions. The new target category is just the simplest symmetric fusion category that contains fermions, namely, the category of super-vector-spaces $s \mathcal{V}$ ec. The fusion part of $s \mathcal{V}$ ec is the same as $\mathcal{R e p}\left(Z_{2}\right)$. But now the nontrivial particle, denoted by $f$ to distinguish from the $\mathcal{R e p}\left(Z_{2}\right)$ example above, is a fermion; its braiding is modified:

$$
c_{f, f}=-\mathrm{id}_{1},
$$

while other braidings remain trivial. It can be understood as vector spaces with a $Z_{2}$ grading. The nontrivial grading corresponds to fermionic d.o.f., while the trivial grading corresponds to bosonic d.o.f.

So when there are fermions in $\mathcal{E}$, we instead need a super-fiber-functor

$$
F: \mathcal{E} \rightarrow \mathrm{s} \mathcal{V e c}
$$

It can be physically realized the same way using the fusion space $\mathcal{V}\left(S^{3}, q, Q\right)$. And we can follow exactly the same procedure introduced in the last subsection to construct a group from automorphisms of the super-fiber-functor $F$,

$$
G_{f} \equiv \operatorname{Aut}(F) .
$$

Such a group is slightly different from the bosonic case. Note that there is a special automorphism $z=\left\{z_{p}\right\}$ : 


$$
z_{p}= \begin{cases}\operatorname{id}_{F(p)}, & p \text { is a boson } \\ -\mathrm{id}_{F(p)}, & p \text { is a fermion }\end{cases}
$$

$z$ corresponds to the fermion number parity and commutes with all other automorphisms. Let $Z_{2}^{f} \equiv\{1, z\}$. We see that the group $G_{f}$ must contain $Z_{2}^{f}$ as a central subgroup. We then have

$$
\mathcal{E} \cong \operatorname{siep}\left(G_{f}\right)
$$

where $\operatorname{s} \mathcal{R e p}\left(G_{f}\right)$ is constructed similarly like $\mathcal{R} \operatorname{ep}(G)$. They have the same fusion; only the braiding between two fermions has an extra -1 . In this sense, we have $\mathrm{s} \mathcal{V e c}=\operatorname{s} \mathcal{R e p}\left(Z_{2}^{f}\right)$.

\section{5. (Super)-fiber-functor from condensation}

In the above, we realize the (super)-fiber-functor using the fusion space on $S^{3}$ with a special background particle $Q$. But we give no proof why such fusion space preserves the fusion and braiding. In this subsection, we explain why such $Q$ is so special. Meanwhile, this subsection also explains why the configuration in Sec. V B gives a superfiber-functor.

In the all-boson case, imagine that we let $Q$ condense to form a new phase, a $Q$-sea, such that $Q$ becomes the trivial particle in the $Q$-sea. As long as the $Q$-sea is a trivial phase, it is guaranteed that we obtain a fiber functor by setting

$$
F(p) \equiv \mathcal{V}\left(S^{3}, p, Q\right)
$$

In short, the requirements for the background particle $Q$ is that it can be condensed to form a trivial phase.

Let us focus on a space $S^{3}$. If we put no excitation on $S^{3}$, there will be no degeneracy both before and after condensation. To relate the $Q$ condensed phase with the fusion space in the uncondensed phase, we split $S^{3}$ into two 3-disks $D_{\text {unc }}^{3}$ and $D_{\text {con }}^{3}$ and condense $Q$ only in $D_{\text {con }}^{3}$. As we discuss in the main text, the domain wall $S_{w}^{2}$ separating two 3-disks can be gapped, such that when no excitation is present there is no degeneracy:

$$
\operatorname{dim} \mathcal{V}\left(S^{3}, D_{\text {unc }}^{3}, S_{w}^{2}, D_{\text {con }}^{3}\right)=1 .
$$

Moreover, since all particles have trivial mutual statistics, such a domain wall $S_{w}^{2}$ is transparent to particles in $D_{\mathrm{unc}}^{3}$. In other words, a particle can move through the domain wall from $D_{\text {unc }}^{3}$ to $D_{\text {con }}^{3}$ without changing degeneracy and introduce only some unitary transformations on the fusion space. In $D_{\text {unc }}^{3}$, the particle carries a group representation; in $D_{\text {con }}^{3}$, as it is the trivial phase whose particles are described by $\mathcal{V e c}$, the particle becomes an accidental degeneracy of trivial particles and is described by a vector space.
Now, consider the fusion space

$$
\mathcal{V}\left[S^{3}, D_{\text {unc }}^{3}(p), S_{w}^{2}, D_{\text {con }}^{3}\right]
$$

where we put a particle $p$ in $D_{\text {unc }}^{3}$. There are three different ways to simplify it.

(1) Shrink $D_{\text {con }}^{3}$ to a point.-As $D_{\text {con }}^{3}$ is the $Q$ condensed phase, whose ground state is a $Q$-sea, this point behaves just like a single particle $Q$ in the uncondensed phase. Thus,

$$
\mathcal{V}\left[S^{3}, D_{\text {unc }}^{3}(p), S_{w}^{2}, D_{\text {con }}^{3}\right] \simeq \mathcal{V}\left(S^{3}, p, Q\right) .
$$

In other words, this fusion space is exactly the vector space $F(p)$ we want to assign to $p$ in the fiber functor.

(2) Shrink $D_{\text {unc }}^{3}(p)$ to a point.-This point should behave like a particle in the condensed phase, which is denoted by $p^{\prime}$. Thus,

$$
\begin{aligned}
\mathcal{V}\left[S^{3}, D_{\mathrm{unc}}^{3}(p), S_{w}^{2}, D_{\mathrm{con}}^{3}\right] & \simeq \mathcal{V}_{\mathrm{con}}\left(S^{3}, p^{\prime}\right) \\
& \simeq \mathcal{V}_{\mathrm{con}}\left(S^{3}, \mathbf{1}, p^{\prime}\right) .
\end{aligned}
$$

Since in the trivial phase, $p^{\prime}$ itself is described by a vector space, we have

$$
p^{\prime} \simeq \mathcal{V}_{\mathrm{con}}\left(S^{3}, \mathbf{1}, p^{\prime}\right)
$$

which implies

$$
p^{\prime} \simeq F(p)
$$

In particular, if $p$ is the trivial particle $\mathbf{1}$, so is $p^{\prime}$ :

$$
\mathcal{V}\left[S^{3}, D_{\text {unc }}^{3}(\mathbf{1}), S_{w}^{2}, D_{\text {con }}^{3}\right] \simeq \mathcal{V}\left(S^{3}, D_{\text {unc }}^{3}, S_{w}^{2}, D_{\text {con }}^{3}\right) \simeq \mathbf{1} .
$$

(3) When there is more than one particle, we also want to deal with them in a more local manner, in order to study fusion and braiding. Thus, we consider the adiabatic process of moving $p$ through the domain wall from the uncondensed phase to the condensed phase. Despite the global topology and background configuration, we obtain a particle in the condensed phase described by the same vector space $p^{\prime} \simeq F(p)$, which can be seen in the special global space $S^{3}$ in the above:

$$
\begin{aligned}
F(p) & \simeq \mathcal{V}\left[S^{3}, D_{\mathrm{unc}}^{3}(p), S_{w}^{2}, D_{\mathrm{con}}^{3}\right] \\
& \simeq p^{\prime} \simeq \mathcal{V}_{\mathrm{con}}\left(S^{3}, \mathbf{1}, p^{\prime}\right) \\
& \simeq \mathcal{V}\left[S^{3}, D_{\mathrm{unc}}^{3}, S_{w}^{2}, D_{\mathrm{con}}^{3}\left(p^{\prime}\right)\right],
\end{aligned}
$$

where we have used the fact that, if we shrink $D_{\text {unc }}^{3}$ to a point, it behaves like a trivial particle in the condensed phase. In other words, we can first move 
$p$ through the domain wall into $D_{\text {con }}^{3}$ and second shrink $D_{\text {unc }}^{3}$ to a point. The resulting particle in $D_{\text {con }}^{3}$ and the resulting fusion space are both $p^{\prime} \simeq F(p)$, the same as shrinking $D_{\text {unc }}^{3}(p)$.

What is important is that moving particles through the domain wall is compatible with fusion:

$$
\begin{aligned}
\mathcal{V} & {\left[S^{3}, D_{\mathrm{unc}}^{3}\left(p_{1} \otimes p_{2}\right), S_{w}^{2}, D_{\mathrm{con}}^{3}\right] } \\
& \simeq \mathcal{V}\left\{S^{3}, D_{\mathrm{unc}}^{3}, S_{w}^{2}, D_{\mathrm{con}}^{3}\left[\left(p_{1} \otimes p_{2}\right)^{\prime}\right]\right\} \\
& \simeq \mathcal{V}\left[S^{3}, D_{\mathrm{unc}}^{3}\left(p_{1}\right), S_{w}^{2}, D_{\mathrm{con}}^{3}\left(p_{2}^{\prime}\right)\right] \\
& \simeq \mathcal{V}\left[S^{3}, D_{\mathrm{unc}}^{3}, S_{w}^{2}, D_{\mathrm{con}}^{3}\left(p_{1}^{\prime} \otimes_{\mathbb{C}} p_{2}^{\prime}\right)\right],
\end{aligned}
$$

and braiding:

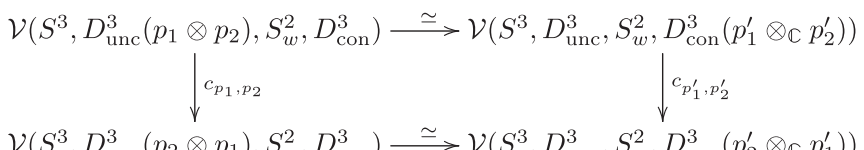

Now, we are ready to prove that $F(p)$ preserves fusion of particles:

$$
\begin{aligned}
F\left(p_{1} \otimes p_{2}\right) & \equiv \mathcal{V}\left(S^{3}, p_{1} \otimes p_{2}, Q\right) \\
& \simeq \mathcal{V}\left[S^{3}, D_{\mathrm{unc}}^{3}\left(p_{1} \otimes p_{2}\right), S_{w}^{2}, D_{\mathrm{con}}^{3}\right] \\
& \simeq \mathcal{V}\left[S^{3}, D_{\mathrm{unc}}^{3}, S_{w}^{2}, D_{\mathrm{con}}^{3}\left(p_{1}^{\prime} \otimes_{\mathbb{C}} p_{2}^{\prime}\right)\right] \\
& \simeq \mathcal{V}_{\mathrm{con}}\left(S^{3}, \mathbf{1}, p_{1}^{\prime} \otimes_{\mathbb{C}} p_{2}^{\prime}\right) \\
& \simeq p_{1}^{\prime} \otimes_{\mathbb{C}} p_{2}^{\prime} \simeq F\left(p_{1}\right) \otimes_{\mathbb{C}} F\left(p_{2}\right) .
\end{aligned}
$$

The property that $F(p)$ also preserves braidings directly follows from Eq. (A49).

If there are emergent fermions, we need to make several modifications to the above discussions.

First, we want similarly a condensate whose particles form sVec. But $Q$ should become, instead of the trivial particle, a direct sum $1 \oplus f$, from whose fusion space we can extract both bosonic and fermionic d.o.f. It turns out $Q$ should be of the following form:

$$
Q=Q_{b} \oplus Q_{f}, \quad \operatorname{dim}\left(Q_{b}\right)=\operatorname{dim}\left(Q_{f}\right),
$$

where $Q_{b}$ and $Q_{f}$ are bosonic and fermionic parts, respectively. We condense the bosonic part $Q_{b}$. Particles above the $Q_{b}$-sea will be sVec, $Q_{b}$ becomes $\mathbf{1}$, and $Q_{f}$ becomes $f$.

Second, in the condensed phase $D_{\text {con }}^{3}$, we need to put $1 \oplus f$ instead of nothing. For super-vector-spaces s $\mathcal{V e c}$, we have the property similar to Eq. (A44):

$$
p^{\prime} \simeq \mathcal{V}_{\text {con }}\left(S^{3}, p^{\prime}, \mathbf{1} \oplus f\right)
$$

Then, we can repeat the above discussions and prove that

$$
F(p) \equiv \mathcal{V}\left(S^{3}, p, Q\right) \simeq \mathcal{V}\left[S^{3}, D_{\mathrm{unc}}^{3}(p), S_{w}^{2}, D_{\mathrm{con}}^{3}(\mathbf{1} \oplus f)\right]
$$

realizes a super-fiber-functor, which is exactly the one we used in Sec. V B. It also has the property that

$$
\begin{aligned}
F(p) & \simeq \mathcal{V}\left[S^{3}, D_{\mathrm{unc}}^{3}(p), S_{w}^{2}, D_{\mathrm{con}}^{3}(\mathbf{1} \oplus f)\right] \\
& \simeq \mathcal{V}\left[S^{3}, D_{\mathrm{unc}}^{3}, S_{w}^{2}, D_{\mathrm{con}}^{3}\left(p^{\prime}, \mathbf{1} \oplus f\right)\right] \\
& \simeq \mathcal{V}_{\mathrm{con}}\left(S^{3}, p^{\prime}, \mathbf{1} \oplus f\right) \simeq p^{\prime}
\end{aligned}
$$

Namely, $F(p)$ is also the super-vector-space describing the particle $p^{\prime}$ resulting from moving $p$ into the condensed phase through the domain wall.

\section{APPENDIX B: RELATION BETWEEN EMERGENT MAJORANA ZERO MODES FOR LINKED LOOPS AND THE 2-COCYCLE $M_{2}$}

In Ref. [65], it is pointed out that, for some fermionic SPT states, certain linked loops of symmetry twists can carry a pair of Majorana zero modes (see Fig. 21). In this Appendix, we discuss a relation between such emergent Majorana zero modes and the nontrivial two cocycle $m_{2}$ that characterize the EF2 topological orders. For simplicity, we assume $G_{f}$ to be Abelian. We show that certain linked looplike excitations in an EF2 topological order carry a pair of Majorana zero modes, one for each linked loop. In other words, certain pairs of looplike excitations carry twofold topological degeneracy when they are linked and no degeneracy when they are not linked. Such a topological degeneracy is highly nonlocal in the sense that the degeneracy is shared between the two well-separated linked loops. The new result here is that the appearance of Majorana zero modes for linked loops is directly related to the nontrivial $Z_{2}^{m}$ extension of $G_{b}$ on the canonical boundary.

To see the above result, we consider a pair of linked loops in the bulk in Fig. 21. We compute the degeneracy for the linked loops. For Abelian $G_{f}$, all the pointlike excitations and stringlike excitations have an unit quantum dimension. Thus, one may expect that degeneracy for the linked loops to be 1 . In the following, we show that sometimes the degeneracy can be 2 . To obtain such a

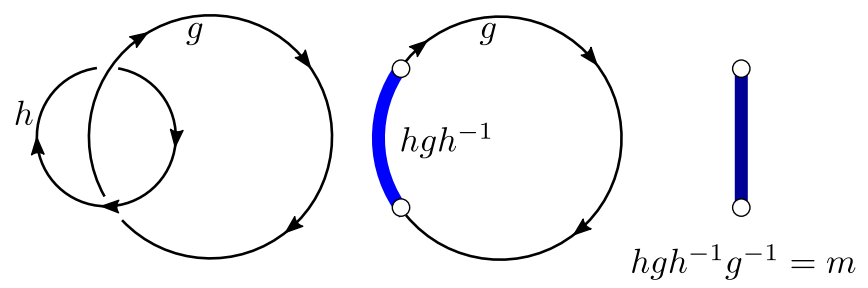

FIG. 21. Fuse $h$ loop to the linked $g$ loop on the canonical domain wall. When $h g h^{-1}=g m$, two Majorana zero modes are supported. Further fusing the two segments, we obtain an open pSC chain. 
result, we bring the linked loops to the boundary, which reduces the group elements $h^{f}, g^{f}$ in $G_{f}$ to the group elements $h^{b}=\pi^{f}\left(h^{f}\right), g^{b}=\pi^{f}\left(g^{f}\right)$ in $G_{b}$ via the projection $G_{f} \stackrel{\pi^{f}}{\rightarrow} G_{b}=G_{f} / Z_{2}^{f}$. In addition to the projection $G_{f} \rightarrow G_{b}$, there is also a lift $G_{b} \rightarrow \hat{G}_{b}$. The linked loops on the boundary are actually described by $h, g$ in $\hat{G}_{b}$, where $h^{b}=$ $\pi^{m}(h), g^{b}=\pi^{m}(g)$ under the projection $\hat{G}_{b} \stackrel{\pi^{m}}{\rightarrow} G_{b}$. To summarize, the bulk string types $h^{f}, g^{f}$ turn to boundary string local types $h, g$ that satisfy the following relation:

$g^{b}=\pi^{f}\left(g^{f}\right)=\pi^{m}(g), \quad h^{b}=\pi^{f}\left(h^{f}\right)=\pi^{m}(h)$.

This situation is described in Fig. 21. As we go around a loop, the boundary string labeled by $g$ turns into a boundary string $h g h^{-1}$. Even though $h^{b}, g^{b}$ commute in $G_{b}$, their lifts $h, g$ may not commute in $\hat{G}_{b}$, when $\hat{G}_{b}$ is a nontrivial $Z_{2}^{m}$ extension of $G_{b}$. If $h, g$ do not commute, we have $h g h^{-1}=g m$, where $m$ generates $Z_{2}^{m}$. As a result, there are two pointlike defects between $g$ and $g m$ boundary strings, corresponding to two Majorana zero modes which lead to a twofold degeneracy.

To see which linked loops described by $h^{f}, g^{f}$ have Majorana zero modes, we first note that the elements in $\hat{G}_{b}$ can be labeled by $\left(g^{b}, x\right), g^{b} \in G_{b}$ and $x \in Z_{2}$. The multiplication in $\hat{G}_{b}$ is given by

$$
\left(g^{b}, x\right)\left(h^{b}, y\right)=\left(g^{b} h^{b}, m_{2}\left(g^{b}, h^{b}\right) x y\right),
$$

where $m_{2}\left(g^{b}, h^{b}\right)$ is the group 2-cocycle in $H^{2}\left(G_{b}, Z_{2}\right)$. For $h^{f}, g^{f}$, we have $h=\left(\pi^{f}\left(h^{f}\right), y\right), g=\left(\pi^{f}\left(g^{f}\right), x\right) \in \hat{G}_{b}$. As shown in Fig. 21, their commutator $[h, g] \equiv h g h^{-1} g^{-1}=$ $h g(g h)^{-1}$ determines the appearance of Majorana zero modes. Using the fact that $h g=[h, g] g h$ and $\pi^{f}\left(h^{f}\right) \pi^{f}\left(g^{f}\right)=\pi^{f}\left(g^{f}\right) \pi^{f}\left(h^{f}\right)$, it is easy to compute $[h, g]=\left(1, m_{2}\left(\pi^{f}\left(h^{f}\right), \pi^{f}\left(g^{f}\right)\right) m_{2}\left(\pi^{f}\left(g^{f}\right), \pi^{f}\left(h^{f}\right)\right)\right)$. We see that the linked loops $h^{f}, g^{f}$ have Majorana zero modes when $m_{2}\left(\pi^{f}\left(h^{f}\right), \pi^{f}\left(g^{f}\right)\right) m_{2}\left(\pi^{f}\left(g^{f}\right), \pi^{f}\left(h^{f}\right)\right)=-1$. The appearance of Majorana zero modes for certain linked loops can detect a certain type of nontrivial $Z_{2}^{m}$ extensions, i.e., those with nontrivial $m_{2}\left(h^{b}, g^{b}\right) m_{2}\left(g^{b}, h^{b}\right)$ for certain pairs of elements $h^{b}, g^{b}$ in $G_{b}$.

[1] D. C. Tsui, H. L. Stormer, and A.C. Gossard, TwoDimensional Magnetotransport in the Extreme Quantum Limit, Phys. Rev. Lett. 48, 1559 (1982).

[2] J. S. Helton, K. Matan, M. P. Shores, E. A. Nytko, B. M. Bartlett, Y. Yoshida, Y. Takano, A. Suslov, Y. Qiu, J.-H. Chung, D. G. Nocera, and Y. S. Lee, Spin Dynamics of the Spin-1/2 Kagome Lattice Antiferromagnet $\mathrm{ZnCu}_{3}(\mathrm{OH})_{6} \mathrm{Cl}_{2}$, Phys. Rev. Lett. 98, 107204 (2007).
[3] Z. Feng, Z. Li, X. Meng, W. Yi, Y. Wei, J. Zhang, Y.-C. Wang, W. Jiang, Z. Liu, S. Li, F. Liu, J. Luo, S. Li, G. q. Zheng, Z. Y. Meng, J.-W. Mei, and Y. Shi, Gapped Spin-1/2 Spinon Excitations in a New Kagome Quantum Spin Liquid Compound Cu $3 \mathrm{Zn}(\mathrm{Oh}) 6$ Fbr, Chin. Phys. Lett. 34, 077502 (2017).

[4] R. Hentrich, A. U. B. Wolter, X. Zotos, W. Brenig, D. Nowak, A. Isaeva, T. Doert, A. Banerjee, P. Lampen-Kelley, D. G. Mandrus, S. E. Nagler, J. Sears, Y.-J. Kim, B. Büchner, and C. Hess, Large Field-Induced Gap of Kitaev-Heisenberg Paramagnons in $\alpha-\mathrm{RuCl}_{3}$, Phys. Rev. Lett. 120, 117204 (2018).

[5] A. U. B. Wolter, L. T. Corredor, L. Janssen, K. Nenkov, S. Schönecker, S.-H. Do, K.-Y. Choi, R. Albrecht, J. Hunger, T. Doert, M. Vojta, and B. Büchner, Field-Induced Quantum Criticality in the Kitaev System $\alpha-\mathrm{RuCl}_{3}$, Phys. Rev. B 96, 041405 (2017).

[6] Z. Wang, S. Reschke, D. Hüvonen, S.-H. Do, K.-Y. Choi, M. Gensch, U. Nage, T. Rõõm, and A. Loidl, Magnetic Excitations and Continuum of a Field-Induced Quantum Spin Liquid in $\alpha-\mathrm{RuCl}_{3}$, Phys. Rev. Lett. 119, 227202 (2017).

[7] X.-G. Wen, Vacuum Degeneracy of Chiral Spin State in Compactified Spaces, Phys. Rev. B 40, 7387 (1989).

[8] X.-G. Wen and Q. Niu, Ground State Degeneracy of the FQH States in Presence of Random Potentials and on High Genus Riemann Surfaces, Phys. Rev. B 41, 9377 (1990).

[9] X. Chen, Z.-C. Gu, and X.-G. Wen, Local Unitary Transformation, Long-Range Quantum Entanglement, Wave Function Renormalization, and Topological Order, Phys. Rev. B 82, 155138 (2010).

[10] C. L. Kane and E. J. Mele, $Z_{2}$ Topological Order and the Quantum Spin Hall Effect, Phys. Rev. Lett. 95, 146802 (2005).

[11] C. Xu and J. E. Moore, Stability of the Quantum Spin Hall Effect: Effects of Interactions, Disorder, and $\mathrm{Z}_{2}$ Topology, Phys. Rev. B 73, 045322 (2006).

[12] C. Wu, B. A. Bernevig, and S.-C. Zhang, Helical Liquid and the Edge of Quantum Spin Hall Systems, Phys. Rev. Lett. 96, 106401 (2006).

[13] J. E. Moore and L. Balents, Topological Invariants of TimeReversal-Invariant Band Structures, Phys. Rev. B 75, 121306 (2007).

[14] R. Roy, Three Dimensional Topological Invariants for Time Reversal Invariant Hamiltonians and the Three Dimensional Quantum Spin Hall Effect, Phys. Rev. B 79, 195322 (2009).

[15] L. Fu, C. L. Kane, and E. J. Mele, Topological Insulators in Three Dimensions, Phys. Rev. Lett. 98, 106803 (2007).

[16] X.-L. Qi, T. Hughes, and S.-C. Zhang, Topological Field Theory of Time-Reversal Invariant Insulators, Phys. Rev. B 78, 195424 (2008).

[17] Z.-C. Gu and X.-G. Wen, Tensor-Entanglement-Filtering Renormalization Approach and Symmetry Protected Topological Order, Phys. Rev. B 80, 155131 (2009).

[18] X. Chen, Z.-X. Liu, and X.-G. Wen, Two-Dimensional Symmetry-Protected Topological Orders and Their Protected Gapless Edge Excitations, Phys. Rev. B 84, 235141 (2011). 
[19] X. Chen, Z.-C. Gu, Z.-X. Liu, and X.-G. Wen, Symmetry Protected Topological Orders and the Group Cohomology of Their Symmetry Group, Phys. Rev. B 87, 155114 (2013).

[20] A. Vishwanath and T. Senthil, Physics of Three Dimensional Bosonic Topological Insulators: Surface Deconfined Criticality and Quantized Magnetoelectric Effect, Phys. Rev. X 3, 011016 (2013).

[21] X.-G. Wen, Colloquium: Zoo of Quantum-Topological Phases of Matter, Rev. Mod. Phys. 89, 041004 (2017).

[22] When no symmetry is specified, the shorthanded term "topological order" in this paper always means the first class without any symmetry, which should be distinguished from the second class that needs symmetry protection.

[23] M. Fannes, B. Nachtergaele, and R. F. Werner, Finitely Correlated States on Quantum Spin Chains, Commun. Math. Phys. 144, 443 (1992).

[24] F. Verstraete, J. I. Cirac, J. I. Latorre, E. Rico, and M. M. Wolf, Renormalization-Group Transformations on Quantum States, Phys. Rev. Lett. 94, 140601 (2005).

[25] X.-G. Wen, Topological Orders in Rigid States, Int. J. Mod. Phys. B 04, 239 (1990).

[26] A. Kitaev, Anyons in an Exactly Solved Model and Beyond, Ann. Phys. (Amsterdam) 321, 2 (2006).

[27] E. Rowell, R. Stong, and Z. Wang, On Classification of Modular Tensor Categories, Commun. Math. Phys. 292, 343 (2009).

[28] X.-G. Wen, A Theory of 2+1D Bosonic Topological Orders, Natl. Sci. Rev. 3, 68 (2016).

[29] T. Lan, L. Kong, and X.-G. Wen, A Theory of $2+1 \mathrm{D}$ Fermionic Topological Orders and Fermionic/Bosonic Topological Orders with Symmetries, Phys. Rev. B 94, 155113 (2016).

[30] G. Moore and N. Read, Nonabelions in the Fractional Quantum Hall Effect, Nucl. Phys. B360, 362 (1991).

[31] X.-G. Wen, Non-Abelian Statistics in the FQH States, Phys. Rev. Lett. 66, 802 (1991).

[32] C. Nayak, S. H. Simon, A. Stern, M. Freedman, and S. D. Sarma, Non-Abelian Anyons and Topological Quantum Computation, Rev. Mod. Phys. 80, 1083 (2008).

[33] X.-G. Wen, Origin of Gauge Bosons from Strong Quantum Correlations (Origin of Light), Phys. Rev. Lett. 88, 011602 (2001).

[34] X.-G. Wen, Quantum Order from String-Net Condensations and Origin of Light and Massless Fermions, Phys. Rev. D 68, 065003 (2003).

[35] M. Levin and X.-G. Wen, Quantum Ether: Photons and Electrons from a Rotor Model, Phys. Rev. B 73, 035122 (2006).

[36] X.-G. Wen, Topological Order: From Long-Range Entangled Quantum Matter to an Unification of Light and Electrons, ISRN Condens. Matter Phys. 2013, 198710 (2013).

[37] T. Lan, L. Kong, and X.-G. Wen, A Classification of $3+1 \mathrm{D}$ Bosonic Topological Orders (I): The Case when Point-like Excitations Are All Bosons, Phys. Rev. X 8, 021074 (2018).

[38] M. Levin and X.-G. Wen, String-Net Condensation: A Physical Mechanism for Topological Phases, Phys. Rev. B 71, 045110 (2005).

[39] K. Walker and Z. Wang, $(3+1)$-TQFTs and Topological Insulators, arXiv:1104.2632.
[40] C. Wang and M. Levin, Braiding Statistics of Loop Excitations in Three Dimensions, Phys. Rev. Lett. 113, 080403 (2014).

[41] S. Jiang, A. Mesaros, and Y. Ran, Generalized Modular Transformations in 3+1D Topologically Ordered Phases and Triple Linking Invariant of Loop Braiding, Phys. Rev. X 4, 031048 (2014).

[42] H. Moradi and X.-G. Wen, Universal Topological Data for Gapped Quantum Liquids in Three Dimensions and Fusion Algebra for Non-Abelian String Excitations, Phys. Rev. B 91, 075114 (2015).

[43] J. Wang and X.-G. Wen, Non-Abelian String and Particle Braiding in Topological Order-Modular sl $(3, z)$ Representation and $3+1 \mathrm{D}$ Twisted Gauge Theory, Phys. Rev. B 91, 035134 (2015).

[44] C.-M. Jian and X.-L. Qi, Layer Construction of 3D Topological States and String Braiding Statistics, Phys. Rev. X 4, 041043 (2014).

[45] P. Ye and Z.-C. Gu, Vortex-Line Condensation in Three Dimensions: A Physical Mechanism for Bosonic Topological Insulators, Phys. Rev. X 5, 021029 (2015).

[46] D. Gaiotto, A. Kapustin, N. Seiberg, and B. Willett, Generalized Global Symmetries, J. High Energy Phys. 02 (2015) 172.

[47] C.-H. Lin and M. Levin, Loop Braiding Statistics in Exactly Soluble Three-Dimensional Lattice Models, Phys. Rev. B 92, 035115 (2015).

[48] P. Ye and Z.-C. Gu, Topological Quantum Field Theory of Three-Dimensional Bosonic Abelian-Symmetry-Protected Topological Phases, Phys. Rev. B 93, 205157 (2016).

[49] J. Wang, X.-G. Wen, and S.-T. Yau, Quantum Statistics and Spacetime Surgery, arXiv:1602.05951.

[50] P. Putrov, J. Wang, and S.-T. Yau, Braiding Statistics and Link Invariants of Bosonic/Fermionic Topological Quantum Matter in $2+1$ and $3+1$ Dimensions, Ann. Phys. (Amsterdam) 384, 254 (2017).

[51] M. Levin, Protected Edge Modes without Symmetry, Phys. Rev. X 3, 021009 (2013).

[52] L. Kong and X.-G. Wen, Braided Fusion Categories, Gravitational Anomalies, and the Mathematical Framework for Topological Orders in Any Dimensions, arXiv:1405 .5858 .

[53] X.-G. Wen, Classifying Gauge Anomalies through SPT Orders and Classifying Gravitational Anomalies through Topological Orders, Phys. Rev. D 88, 045013 (2013).

[54] L. Kong, X.-G. Wen, and H. Zheng, Boundary-Bulk Relation for Topological Orders as the Functor Mapping Higher Categories to Their Centers, arXiv:1502.01690.

[55] L. Kong, X.-G. Wen, and H. Zheng, Boundary-Bulk Relation in Topological Orders, Nucl. Phys. B922, 62 (2017).

[56] P. Deligne, Catégories Tensorielles (Tensor Categories), Moscow Math. J. 2, 227 (2002).

[57] C. Zhu, T. Lan, and X.-G. Wen, Topological Non-Linear $\sigma$-Model, Higher Gauge Theory, and a Realization of All $3+1 \mathrm{D}$ Topological Orders for Boson Systems, arXiv: 1808.09394.

[58] M. Cheng, N. Tantivasadakarn, and C. Wang, Loop Braiding Statistics and Interacting Fermionic SymmetryProtected Topological Phases in Three Dimensions, Phys. Rev. X 8, 011054 (2018). 
[59] L. H. Kauffman and R. A. Baadhio, A Categorical Construction of 4d Topological Quantum Field Theories, Quantum Topology (World Scientific, Singapore, 1993), p. 120.

[60] L. H. Kauffman and R. A. Baadhio, Evaluating the CraneYetter Invariant, Quantum Topology (World Scientific, Singapore, 1993), p. 131.

[61] L. Crane, L. H. Kauffman, and D. N. Yetter, State-Sum Invariants of 4-Manifolds I, J. Knot Theory Ramifications 6 , 177 (1997).

[62] A. Kapustin and R. Thorngren, Higher Symmetry and Gapped Phases of Gauge Theories, arXiv:1309.4721.

[63] A. Y. Kitaev, Unpaired Majorana Fermions in Quantum Wires, Phys. Usp. 44, 131 (2001).

[64] L. S. Levitov, T. P. Orlando, J. B. Majer, and J. E. Mooij, Quantum Spin Chains and Majorana States in Arrays of Coupled Qubits, arXiv:cond-mat/0108266.

[65] C.-J. Wang (private communication).

[66] N. Steenrod, Products of Cocycles and Extensions of Mappings, Ann. Math. 48, 290 (1947).

[67] Z.-C. Gu and X.-G. Wen, Symmetry-Protected Topological Orders for Interacting Fermions-Fermionic Topological Non-Linear Sigma-Models and a Group Super-Cohomology Theory, Phys. Rev. B 90, 115141 (2014).

[68] T. Lan, C. Zhu, and X.-G. Wen, Fermion Decoration Construction of Symmetry Protected Trivial Orders for Fermion Systems with Any Symmetries $G_{-} f$ and in Any Dimensions, arXiv:1809.01112.

[69] In the braided fusion 2-category describing the bulk excitations, $\mathcal{A}_{i}$ are just the 1 -morphisms on the object $i$, $\mathcal{A}_{i}=\operatorname{Hom}(i, i)$.

[70] A. Davydov, M. Müger, D. Nikshych, and V. Ostrik, The Witt Group of Non-Degenerate Braided Fusion Categories, J. Reine Angew. Math. (Crelles J.) 2013, 135 (2013).

[71] A. Kapustin, Bosonic Topological Insulators and Paramagnets: A View from Cobordisms, arXiv:1404.6659.

[72] D. S. Freed, Short-Range Entanglement and Invertible Field Theories, arXiv:1406.7278.

[73] M. Levin and X.-G. Wen, Fermions, Strings, and Gauge Fields in Lattice Spin Models, Phys. Rev. B 67, 245316 (2003).

[74] C. W. von Keyserlingk, F. J. Burnell, and S. H. Simon, Three-Dimensional Topological Lattice Models with Surface Anyons, Phys. Rev. B 87, 045107 (2013).

[75] X.-G. Wen, Exactly Soluble Local Bosonic Cocycle Models, Statistical Transmutation, and Simplest Time-Reversal Symmetric Topological Orders in 3+1D, Phys. Rev. B 95, 205142 (2017).

[76] L. Kong, Anyon Condensation and Tensor Categories, Nucl. Phys. B886, 436 (2014).
[77] Without such a background particle, the fusion space would be 0 if $p$ is a fermion. See Appendix A 5 for a more detailed explanation.

[78] N. Gurski, An Algebraic Theory of Tricategories (unpublished).

[79] Q.-R. Wang and Z.-C. Gu, Towards a Complete Classification of Symmetry-Protected Topological Phases for Interacting Fermions in Three Dimensions and a General Group Supercohomology Theory, Phys. Rev. X 8, 011055 (2018).

[80] A. Kapustin and R. Thorngren, Fermionic SPT Phases in Higher Dimensions and Bosonization, J. High Energy Phys. 10 (2017) 080.

[81] X.-G. Wen and A. Zee, A Classification and Matrix Formulation of the Abelian FQH States, Phys. Rev. B 46, 2290 (1992).

[82] X.-G. Wen, Topological Orders and Edge Excitations in FQH States, Adv. Phys. 44, 405 (1995).

[83] G. Brumfiel and J. Morgan, The Pontrjagin Dual of 4-Dimensional Spin Bordism, arXiv:1803.08147.

[84] Q.-R. Wang and Z.-C. Gu, Construction and Classification of Symmetry Protected Topological Phases in Interacting Fermion Systems, arXiv:1811.00536.

[85] J. Klassen and X.-G. Wen, Topological Degeneracy (Majorana Zero-Mode) and $1+1 \mathrm{D}$ Fermionic Topological Order in a Magnetic Chain on Superconductor via Spontaneous $Z_{2}^{f}$ Symmetry Breaking, J. Phys. Condens. Matter 27, 405601 (2015).

[86] T. Lan, L. Kong, and X.-G. Wen, Modular Extensions of Unitary Braided Fusion Categories and $2+1 \mathrm{D}$ Topological/SPT Orders with Symmetries, Commun. Math. Phys. 351, 709 (2017).

[87] T. Lan, L. Kong, and X.-G. Wen, Classification of $(2+1)$-Dimensional Topological Order and SymmetryProtected Topological Order for Bosonic and Fermionic Systems with On-Site Symmetries, Phys. Rev. B 95, 235140 (2017).

[88] T. Tannaka, Über den Dualitiätssatz der Nichtkommutativen Topologischen Gruppen, Tohoku Math. J. 45, 1 (1938).

[89] In the mathematical formulation of fusion categories, this $p^{\prime} \rightarrow p$ morphism is only an embedding morphism; generic morphisms are generated by linear combinations, compositions, taking Hermitian conjugates of these embeddings. But in physics we always (secretly) assume linearity and unitarity (including taking Hermitian conjugates of morphisms). So taking only the embedding as our (basis) morphism suffices for our purpose.

Correction: The abstract contained a typesetting error and has been fixed; the Popular Summary has been modified for clarity. 